\title{
Isolated Organometallic Nickel(III) and Nickel(IV) Complexes Relevant to Carbon-Carbon Bond Formation Reactions
}

Jason W. Schultz, ${ }^{\dagger}$ Kei Fuchigami,${ }^{\dagger}$ Bo Zheng, ${ }^{\dagger}$ Nigam P. Rath,${ }^{\ddagger}$ and Liviu M. Mirica ${ }^{*} \dagger$

$\dagger$ Department of Chemistry, Washington University, One Brookings Drive, St. Louis, Missouri 63130-4899, United States

$\$$ Department of Chemistry and Biochemistry, University of Missouri-St. Louis, One University Boulevard, St. Louis, Missouri 63121-4400, United States

*e-mail: mirica@wustl.edu

\section{Table of Contents}

I. General Specifications

II. Synthesis of Ni Complexes

Preparation of $\left({ }^{\mathrm{Me}} \mathrm{N} 4\right) \mathrm{Ni}^{\mathrm{II}} \mathrm{Br}_{2}$

Preparation of $\left({ }^{\mathrm{Me}} \mathrm{N} 4\right) \mathrm{Ni}^{\mathrm{II}} \mathrm{Me}_{2}, 1$

Preparation of $\left[\left({ }^{\mathrm{Me}} \mathrm{N} 4\right) \mathrm{Ni}^{\mathrm{III}} \mathrm{Me}_{2}\right] \mathrm{PF}_{6}, 2$

Preparation of $\left({ }^{\mathrm{Me}} \mathrm{N} 4\right) \mathrm{Ni}^{\mathrm{II}}\left(\mathrm{CD}_{3}\right)_{2}$

Preparation of $\left({ }^{\mathrm{Me}} \mathrm{N} 4\right) \mathrm{Ni}^{\mathrm{II}}$ (cycloneophyl) , 5

Preparation of $\left({ }^{\mathrm{Me}} \mathrm{N} 4\right) \mathrm{Ni}^{\mathrm{III}}$ (cycloneophyl)PF 6,6

Preparation of $\left({ }^{\mathrm{Me}} \mathrm{N} 4\right) \mathrm{Ni}^{\mathrm{iV}}$ (cycloneophyl) $\left(\mathrm{PF}_{6}\right)_{2}, 7$

III. Cyclic voltammograms of Isolated Ni Complexes

IV. UV-Vis spectra of Isolated Ni Complexes

V. Simulation of EPR spectra of Isolated $\mathrm{Ni}^{\mathrm{III}}$ Complexes

VI. NMR spectra of $\mathrm{Ni}^{\mathrm{IV}}$ Complex

VII. XPS of Isolated Ni Complexes

VIII. Computational Details

IX. Reactivity of Ni Complexes

$X$. Catalytic Kumada Coupling Reactions

XI. X-ray structure determinations of Ni Complexes 


\section{General specifications}

All manipulations were carried out under a nitrogen atmosphere using standard Schlenk and glove box techniques if not indicated otherwise. All reagents for which synthesis is not given were commercially available from Aldrich, Acros, STREM or Pressure Chemical and were used as received without further purification. Solvents were purified prior to use by passing through a column of activated alumina using an MBRAUN SPS. $\operatorname{Mg}\left(\mathrm{CD}_{3}\right) \mathrm{I}^{1}{ }^{1} \mathrm{~N}, \mathrm{~N}$ '-methyl-2,11diaza[3,3] $(2,6)$ pyridinophane $\left({ }^{\mathrm{Me}} \mathrm{N} 4\right),{ }^{2}\left(\mathrm{PMe}_{3}\right)_{2} \mathrm{Ni}^{\mathrm{II}}$ (cycloneophyl), ${ }^{3}$ and $\mathrm{FcPF}_{6}{ }^{4}$ were prepared according to the literature procedures.

${ }^{1} \mathrm{H}$ NMR spectra were recorded on a Varian Mercury-300 spectrometer $(300.121 \mathrm{MHz})$, Agilent DD2-500 spectrometer (499.885 MHz) or Agilent DD2-600 spectrometer (599.736 $\mathrm{MHz}$ ). Chemical shifts are reported in ppm and referenced to residual solvent resonance peaks. ${ }^{5}$ Abbreviations for the multiplicity of NMR signals are s (singlet), d (doublet), $t$ (triplet), $q$ (quartet), m (multiplet), br (broad). UV-visible spectra were recorded on a Varian Cary 50 Bio spectrophotometer and are reported as $\lambda_{\max }, \mathrm{nm}\left(\varepsilon, \mathrm{M}^{-1 *} \mathrm{~cm}^{-1}\right)$. EPR spectra were recorded on a Bruker EMX-PLUS EPR or a JEOL JES-FA EPR spectrometer at X-band ( $9.2 \mathrm{GHz})$ frequency in frozen solution at $77 \mathrm{~K}$. The purchase of the Bruker EMX-PLUS EPR spectrometer was supported by the National Science Foundation (MRI, CHE-1429711). ESI-MS experiments were performed using a Thermo FT or Bruker Maxis Q-TOF mass spectrometer with an electrospray ionization source. ESI mass-spectrometry was provided by the Washington University Mass Spectrometry Resource, an NIH Research Resource (Grant No. P41RR0954). Elemental analyses were carried out by the Intertek Pharmaceutical Services.

Electrochemical-grade electrolytes from Fluka were used as the supporting electrolyte for electrochemical measurements. Cyclic voltammetry and controlled potential electrolysis experiments were performed with a BASi EC Epsilon electrochemical workstation or a CHI 660D Electrochemical Analyzer. The electrochemical measurements were performed under a blanket of nitrogen, and the analyzed solutions were deaerated by purging with nitrogen. A glassy carbon disk electrode $(d=1.6 \mathrm{~mm})$ was used as the working electrode for cyclic voltammetry. The auxiliary electrode was a Pt wire for cyclic voltammetry measurements. Two different non-aqueous references electrodes were used: $\mathrm{Ag} /$ filled with $0.01 \mathrm{M} \mathrm{AgNO}_{3} / 0.1 \mathrm{M}$ $\mathrm{Bu}_{4} \mathrm{NClO}_{4} / \mathrm{MeCN}$ solution or silver wire. The reference electrodes were calibrated against $\mathrm{Cp}_{2} \mathrm{Fe}(\mathrm{Fc})$. 


\section{II.Synthesis of Ni Complexes}

\section{Preparation of ( $\left.{ }^{\mathrm{Me}} \mathrm{N} 4\right) \mathrm{Ni}^{\mathrm{II}} \mathrm{Br}_{2}$.}

$\left({ }^{\mathrm{Me}} \mathrm{N} 4\right)+(\mathrm{DME}) \mathrm{NiBr}_{2} \stackrel{\mathrm{DCM}}{\longrightarrow}\left({ }^{\mathrm{Me}} \mathrm{N} 4\right) \mathrm{NiBr}_{2}$

$(\mathrm{DME}) \mathrm{NiBr}_{2}(0.345 \mathrm{~g}, 1.12 \mathrm{mmol})$ and ${ }^{\mathrm{Me}} \mathrm{N} 4(0.300$ grams, $1.12 \mathrm{mmol})$ were dissolved in $60 \mathrm{ml}$ of $\mathrm{CH}_{2} \mathrm{Cl}_{2}$ at RT under $\mathrm{N}_{2}$. The solution turned from orange to green. After 24 hours, the solvent was evaporated under vacuum and the solid residue was recrystallized from $\mathrm{CH}_{2} \mathrm{Cl}_{2}$ with slow ether diffusion to afford green paramagnetic crystals (0.341 grams, $0.70 \mathrm{mmol}, 63 \%)$.

UV-Vis, $\lambda$, nm ( $\left.\varepsilon, \mathrm{M}^{-1} \cdot \mathrm{cm}^{-1}\right)$, DCM: 259 (2305), 315 (sh, 680), 405 (281), 623 (22), 980 (33).

Evans method $\left(\mathrm{CD}_{3} \mathrm{CN}\right): \mu_{\mathrm{eff}}=3.05 \mu_{\mathrm{b}}$.

${ }^{1} \mathrm{H}-\mathrm{NMR}$ (300 MHz, $\mathrm{CDCl}_{3}$ ), $\delta$ (ppm): 68.45 (br), 56.41 (br), 13.23 (br), 1.58 (br).

Anal. Found: C, 37.18; H, 4.03; N, 10.41. Calculated for $\mathrm{C}_{16} \mathrm{H}_{20} \mathrm{Br}_{2} \mathrm{~N}_{4} \mathrm{Ni} \cdot 1 / 2 \mathrm{CH}_{2} \mathrm{Cl}_{2}$ : C, 37.44; H, 4.00; N, 10.58 .

\section{Preparation of ( $\left.{ }^{\mathrm{Me}} \mathrm{N}\right) \mathrm{Ni}^{\mathrm{II}} \mathrm{Me}_{2}, 1$.}

$\left({ }^{\mathrm{Me}} \mathrm{N} 4\right) \mathrm{NiBr}_{2}+\mathrm{MeMgCl} \stackrel{\mathrm{THF}}{\longrightarrow}\left({ }^{\mathrm{Me}} \mathrm{N} 4\right) \mathrm{NiMe}_{2}$

To a stirred suspension of $\left({ }^{\mathrm{Me}} \mathrm{N} 4\right) \mathrm{NiBr}_{2}(177 \mathrm{mg}, 0.364 \mathrm{mmol})$ in $\mathrm{THF}(5 \mathrm{~mL})$, cooled at $-50^{\circ} \mathrm{C}$ under $\mathrm{N}_{2}$, was added $0.56 \mathrm{~mL}$ of a ca. $1.94 \mathrm{M}$ solution of $\mathrm{MeMgCl}$ in $\mathrm{THF}$ solution. The mixture was stirred at this temperature for $30 \mathrm{~min}$, and then slowly warmed up to $-5^{\circ} \mathrm{C}$ overnight. Then dioxane $(5 \mathrm{~mL})$ was added to the deep red solution. The resulting suspension was let stand for 1 hour in the $-35^{\circ} \mathrm{C}$ freezer. Then the suspension was filtered and the solvent was completely removed under vacuum at $-50^{\circ} \mathrm{C}$ and the residue was extracted with precooled diethyl ether (100 $\mathrm{mL}$ ). The deep red solution was filtered and dried under vacuum. A red solid was obtained $(84.1 \mathrm{mg}, 0.235 \mathrm{mmol}, 65 \%)$. X-ray quality crystals were obtained by slow pentane diffusion into a diethyl ether solution at $-35^{\circ} \mathrm{C}$. Several attempts to obtain elemental analysis data proved unsuccessful, likely due to the oxidation of $\mathbf{1}$ during transportation and handling.

UV-Vis, $\lambda, \mathrm{nm}\left(\varepsilon, \mathrm{M}^{-1} \cdot \mathrm{cm}^{-1}\right)$, THF: 400 (3100).

${ }^{1} \mathrm{H}-\mathrm{NMR}\left(300 \mathrm{MHz}, \mathrm{d}_{3}-\mathrm{MeCN}\right), \delta$ (ppm): 7.42 (t, 2H, Py-H), 7.00 (d, 4H, Py-H), 6.77 (d, 2H, $\left.\mathrm{CH}_{2}-\right), 4.22\left(\mathrm{~d}, 2 \mathrm{H},-\mathrm{CH}_{2}-\right), 2.25\left(\mathrm{~s}, 18 \mathrm{H},-\mathrm{CH}_{3}\right),-0.91\left(\mathrm{~s}, 6 \mathrm{H}, \mathrm{Ni}-\mathrm{CH}_{3}\right)$.

${ }^{13} \mathrm{C}-\mathrm{NMR}$ (600 MHz, d8-THF), $\delta$ (ppm): 159.22, 134.46, 124.58, 63.94, 63.94, 38.48, -9.65. 


\section{Preparation of $\left[\left({ }^{\mathrm{Me}} \mathrm{N} 4\right) \mathrm{Ni}^{\mathrm{III}} \mathrm{Me}_{2}\right] \mathrm{PF}_{6}, 2$.}

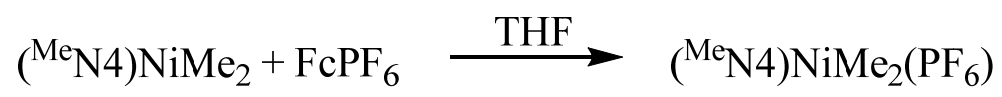

A solution of 1 (32.8 mg, $0.092 \mathrm{mmol})$ and $\mathrm{FcPF}_{6}(30.4 \mathrm{mg}, 0.092 \mathrm{mmol})$ in pre-cooled THF was stirred at $-50{ }^{\circ} \mathrm{C}$ for 3 hours under $\mathrm{N}_{2}$ and then resulting solution was then filtered at $-50{ }^{\circ} \mathrm{C}$. To the filtrate was added pre-cooled pentane producing a precipitated immediately. After filtration, an orange-red solid was isolated and dried under vacuum $(17.6 \mathrm{mg}, 0.035 \mathrm{mmol}, 38 \%)$. X-ray quality crystals were obtained by slow pentane diffusion into a tetrahydrofuran solution at $-35^{\circ} \mathrm{C}$ in the presence of $\mathrm{NaBPh}_{4}$. Elemental analysis sample was obtained using similar X-ray quality crystals obtained under the same conditions.

Evans method $\left(\mathrm{CD}_{3} \mathrm{CN}\right): \mu_{\mathrm{eff}}=1.70 \mu \mathrm{b}$.

UV-Vis, $\lambda, \mathrm{nm}\left(\varepsilon, \mathrm{M}^{-1} \cdot \mathrm{cm}^{-1}\right)$, MeCN: 240 (10800), 465 (470).

ESI-MS (HR) in Acetonitrile: m/z 356.1501; Calculated for [( $\left.{ }^{\mathrm{Me} N 4) \mathrm{Ni}^{\mathrm{III}} \mathrm{Me}}\right]^{+}: \mathrm{m} / \mathrm{z} 356.1511$. Anal. Found: C, 73.31; H, 6.89; N, 7.55. Calculated for $\mathrm{C}_{42} \mathrm{H}_{46} \mathrm{BN}_{4} \mathrm{Ni}^{-} \mathrm{C}_{4} \mathrm{H}_{8} \mathrm{O}$ : C, 73.82; H, 7.27; N, 7.49.

\section{Preparation of $\left({ }^{\mathrm{Me}} \mathrm{N} 4\right) \mathrm{Ni}^{\mathrm{II}}\left(\mathrm{CD}_{3}\right)_{2}, 1-\mathrm{d}_{6}$.}

$$
\left({ }^{\mathrm{BBu}} \mathrm{N} 4\right) \mathrm{NiBr}_{2}+\mathrm{CD}_{3} \mathrm{MgCl} \stackrel{\mathrm{THF}}{\longrightarrow}\left({ }^{\mathrm{BBu}} \mathrm{N} 4\right) \mathrm{Ni}\left(\mathrm{CD}_{3}\right)_{2}
$$

To a stirred suspension of $\left({ }^{\mathrm{Me}} \mathrm{N} 4\right) \mathrm{NiBr}_{2}(220 \mathrm{mg}, 0.452 \mathrm{mmol})$ in THF $(6 \mathrm{~mL})$, cooled at $-50^{\circ} \mathrm{C}$, was added $1.35 \mathrm{~mL}$ of the $\mathrm{CD}_{3} \mathrm{MgI}$ solution. The mixture was stirred at this temperature for 30 min, and then slowly warmed up to $-5^{\circ} \mathrm{C}$ overnight. Then dioxane $(6 \mathrm{~mL})$ was added to the deep red solution. The resulting suspension was let stand for 1 hour in the $-35^{\circ} \mathrm{C}$ freezer. Then the suspension was filtered and the solvent was completely removed under vacuum and the residue was extracted with precooled diethyl ether $(150 \mathrm{~mL})$. The deep red solution was filtered and dried under vacuum. A red solid was obtained $(150 \mathrm{mg}, 0.413 \mathrm{mmol}, 91 \%)$. The ${ }^{1} \mathrm{H}$ NMR spectrum of the product is identical to that of $\left({ }^{\mathrm{Me}} \mathrm{N} 4\right) \mathrm{Ni}^{\mathrm{II}} \mathrm{Me}_{2}$ except for the missing singlet for the Ni-Me group. 


\section{Preparation of ( $\left.{ }^{\mathrm{Me}} \mathrm{N} 4\right) \mathrm{Ni}^{\mathrm{iI}}(\mathrm{cycloneophyl}), 5$.}

$\left({ }^{\mathrm{Me}} \mathrm{N} 4\right)+\left(\mathrm{PMe}_{3}\right) \mathrm{Ni}($ cycloneophyl $) \stackrel{\mathrm{Et}_{2} \mathrm{O}}{\longrightarrow}\left({ }^{\mathrm{Me}} \mathrm{N} 4\right) \mathrm{Ni}$ (cycloneophyl)

A solution of ${ }^{\mathrm{Me}} \mathrm{N} 4(0.2300$ grams, $0.86 \mathrm{mmol})$ and $\left(\mathrm{PMe}_{3}\right)_{2} \mathrm{Ni}^{\mathrm{II}}$ (cycloneophyl) $(0.2940$ grams, $0.86 \mathrm{mmol}$ ) in diethyl ether was stirred at room temperature overnight. The red precipitate was then separated by filtration, washed with pentane $(3 \mathrm{~mL})$, and dried under vacuum. Yield: 0.2473 grams, $0.54 \mathrm{mmol}, 63 \%$.

UV-vis (MeCN, RT), $\lambda_{\max }\left(\varepsilon, \mathrm{M}^{-1} \mathrm{~cm}^{-1}\right): 247$ (17500), 302 (5900), 367 (5530), 490 (350).

${ }^{1} \mathrm{H}-\mathrm{NMR}(300 \mathrm{MHz}, \mathrm{CDCl} 3), \delta$ (ppm): 7.54 (t, 1H, Py-H), 7.46 (t, 1H, Py-H), 7.11 (d, 2H, PyH), 7.04 (d, 2H, Py-H), 6.66 (m, 6H, Cycle/- $\left.\mathrm{CH}_{2}-\right), 6.38$ (t, 1H, Cycle), 5.86 (d, 1H, Cycle), 4.22 (dd, 4H, - $\mathrm{CH}_{2}-$ ), 2.24 (s, 6H, N-CH3), 1.32 (s, 6H, CH3), 1.24 (s, 2H, Ni-CH2-Cycle).

${ }^{13} \mathrm{C}-\mathrm{NMR}(600 \mathrm{MHz}, \mathrm{d} 3-\mathrm{MeCN}), \delta$ (ppm): 159.21, 158.49, 136.35, 136.16, 136.05, 135.88, $125.59,125.48,123.89,123.24,122.95,121.91,66.70,64.07,63.95,49.09,38.87,34.21$.

Several attempts to obtain elemental analysis data proved unsuccessful, likely due to the oxidation of 5 during transportation and handling.

Preparation of $\left[\left({ }^{\mathrm{Me}} \mathrm{N} 4\right) \mathrm{Ni}{ }^{\mathrm{II}}(\right.$ cycloneophyl $\left.)\right] \mathrm{PF}_{6}, 6$.

$\left({ }^{\mathrm{Me}} \mathrm{N} 4\right) \mathrm{Ni}$ (cycloneophyl) $+\mathrm{FcPF}_{6} \stackrel{\mathrm{THF}}{\longrightarrow}\left({ }^{\mathrm{Me}} \mathrm{N} 4\right) \mathrm{Ni}\left(\right.$ cycloneophyl) $\mathrm{PF}_{6}$

A THF solution of $\left({ }^{\mathrm{Me}} \mathrm{N} 4\right) \mathrm{Ni}^{\mathrm{II}}$ (cycloneophyl) (0.1581 grams, $\left.0.34 \mathrm{mmol}\right)$ and $\mathrm{FcPF} 6(0.1140$ grams, $0.34 \mathrm{mmol}$ ) was stirred at $-50{ }^{\circ} \mathrm{C}$ for 2 hours. The resulting solution was then precipitated with excess pentane. The orange precipitate was separated by filtration, washed with pentane (3 $\mathrm{mL}$ ), and dried under vacuum. Yield: 0.1658 grams, $0.27 \mathrm{mmol}, 79 \%$. X-ray quality crystals were obtained by slow pentane diffusion into a tetrahydrofuran solution in the presence of $\mathrm{NaBPh}_{4}$ at $-35^{\circ} \mathrm{C}$.

Evans method $\left(\mathrm{CD}_{3} \mathrm{CN}\right): \mu_{\mathrm{eff}}=1.894 \mu \mathrm{b}$.

UV-vis (MeCN, RT), $\lambda_{\max }\left(\varepsilon, \mathrm{M}^{-1} \mathrm{~cm}^{-1}\right): 246$ (18000), 473 (630), 964 (50).

ESI-MS of $\quad\left[\left({ }^{\mathrm{Me}} \mathrm{N} 4\right) \mathrm{Ni}^{\mathrm{III}}(\right.$ cycloneophyl $\left.)\right] \mathrm{PF}_{6}, \quad \mathrm{~m} / \mathrm{z} \quad 458.1969 ;$ calculated for $\left[\left({ }^{\mathrm{Me}} \mathrm{N} 4\right) \mathrm{Ni}{ }^{\mathrm{III}} \text { (cycloneophyl) }\right]^{+}, 458.1980$.

Anal. Found: C, 53.32; H, 5.79; N, 7.96. Calculated for $\mathrm{C}_{26} \mathrm{H}_{32} \mathrm{~F}_{6} \mathrm{~N}_{4} \mathrm{NiP}^{-} \mathrm{C}_{4} \mathrm{H}_{8} \mathrm{O}$ : C, 53.28; H, $5.96 ; \mathrm{N}, 8.28$. 


\section{Preparation of $\left[\left({ }^{\mathrm{Me}} \mathrm{N} 4\right) \mathrm{Ni}^{\mathrm{IV}}(\right.$ cycloneophyl) $]\left(\mathrm{PF}_{6}\right)_{2}, 7$.}

$$
\left({ }^{\mathrm{Me}} \mathrm{N} 4\right) \mathrm{Ni} \text { (cycloneophyl) } \mathrm{PF}_{6}+\mathrm{NOPF}_{6} \stackrel{\mathrm{MeCN}}{\longrightarrow}\left({ }^{\mathrm{Me}} \mathrm{N} 4\right) \mathrm{Ni}(\text { cycloneophyl)PF} 6
$$

A d3-MeCN solution of [ $\left({ }^{\mathrm{Me}} \mathrm{N} 4\right) \mathrm{Ni}^{\mathrm{III}}$ (cycloneophyl) $] \mathrm{PF}_{6}(20 \mathrm{mg}, 0.03 \mathrm{mmol})$ and $\mathrm{NOPF}_{6}(29 \mathrm{mg}$, $0.17 \mathrm{mmol}$ ) was stirred at $-40{ }^{\circ} \mathrm{C}$ for 15 minutes. The resulting orange-red solution was then transferred to a pre-cooled NMR tube. NMR experiments were conducted at $-15^{\circ} \mathrm{C}$. An X-ray structure was not obtained due to decomposition of X-ray quality crystals during data collection. X-ray quality crystals were however obtained by slow pentane diffusion into a tetrahydrofuran solution at $-35^{\circ} \mathrm{C}$. Elemental analysis proved unsuccessful due to instability and decomposition of the complex.

UV-vis (MeCN, RT), $\lambda_{\max }\left(\varepsilon, \mathrm{M}^{-1} \mathrm{~cm}^{-1}\right): 258$ (8825), 305 (1400), 457 (525).

${ }^{1} \mathrm{H}-\mathrm{NMR}\left(500 \mathrm{MHz}, \mathrm{CDCl} 3,-15^{\circ} \mathrm{C}\right), \delta(\mathrm{ppm}): 8.05$ (t, 1H, Py-H), 7.97 (t, 1H, Py-H), 7.51 (d, 2H, Py-H), 7.41 (d, 2H, Py-H), 7.25 (t, 1H, Cyc-H), 7.19 (d, 1H, Cyc-H), 6.92 (t, 1H, Cyc-H), $6.55(\mathrm{~d}, 1 \mathrm{H}, \mathrm{Cyc}-\mathrm{H}), 5.33\left(\mathrm{~s}, 2 \mathrm{H}, \mathrm{Ni}-\mathrm{CH}_{2}-\mathrm{Cyc}\right), 5.01\left(\mathrm{~d}, 2 \mathrm{H},-\mathrm{CH}_{2}-\right), 4.56\left(\mathrm{~d}, 2 \mathrm{H},-\mathrm{CH}_{2}-\right), 4.22(\mathrm{~d}$, $\left.2 \mathrm{H},-\mathrm{CH}_{2}-\right), 4.08$ (d, 2H, - $\left.\mathrm{CH}_{2}-\right), 2.38\left(\mathrm{~s}, 6 \mathrm{H}, \mathrm{N}-\mathrm{CH}_{3}\right), 1.67$ (s, 6H, - $\left.\mathrm{CH}_{3}\right)$.

${ }^{13} \mathrm{C}-\mathrm{NMR}\left(500 \mathrm{MHz}, \mathrm{CDCl} 3,-15^{\circ} \mathrm{C}\right), \delta(\mathrm{ppm}): 159.42,158.44,156.25,154.50,144.89,133.85$, $132.31,131.31,131.15,125.82,125.07,79.77,77.38,57.31,36.00$. 


\section{Cyclic Voltammograms (CVs) of Isolated Ni Complexes}
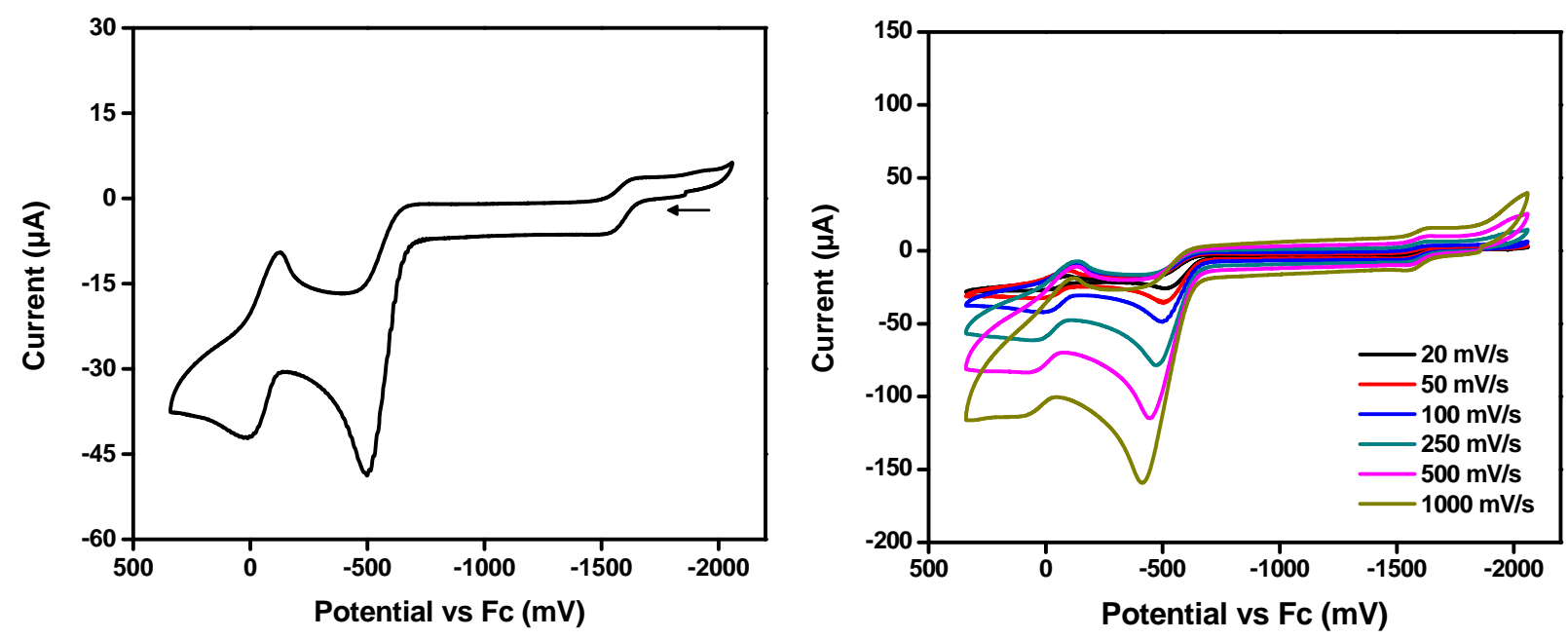

Figure S1: CVs of $\left({ }^{\mathrm{Me}} \mathrm{N} 4\right) \mathrm{NiMe}_{2}$ in $\mathrm{MeCN}$ at $\mathrm{RT}\left(\left[\mathrm{Bu}_{4} \mathrm{NPF}_{6}\right]=0.1 \mathrm{M}\right.$, glassy carbon disk electrode), at $100 \mathrm{mV} / \mathrm{s}$ scan rate (left) and variable scan rates (right). Potentials vs $\mathrm{Fc} / \mathrm{Fc}^{+}(\mathrm{V})$ : $E_{p a}=-1.50,-0.50$ and $0.03, E_{p c}=-0.10,-0.70$ and -1.65 .
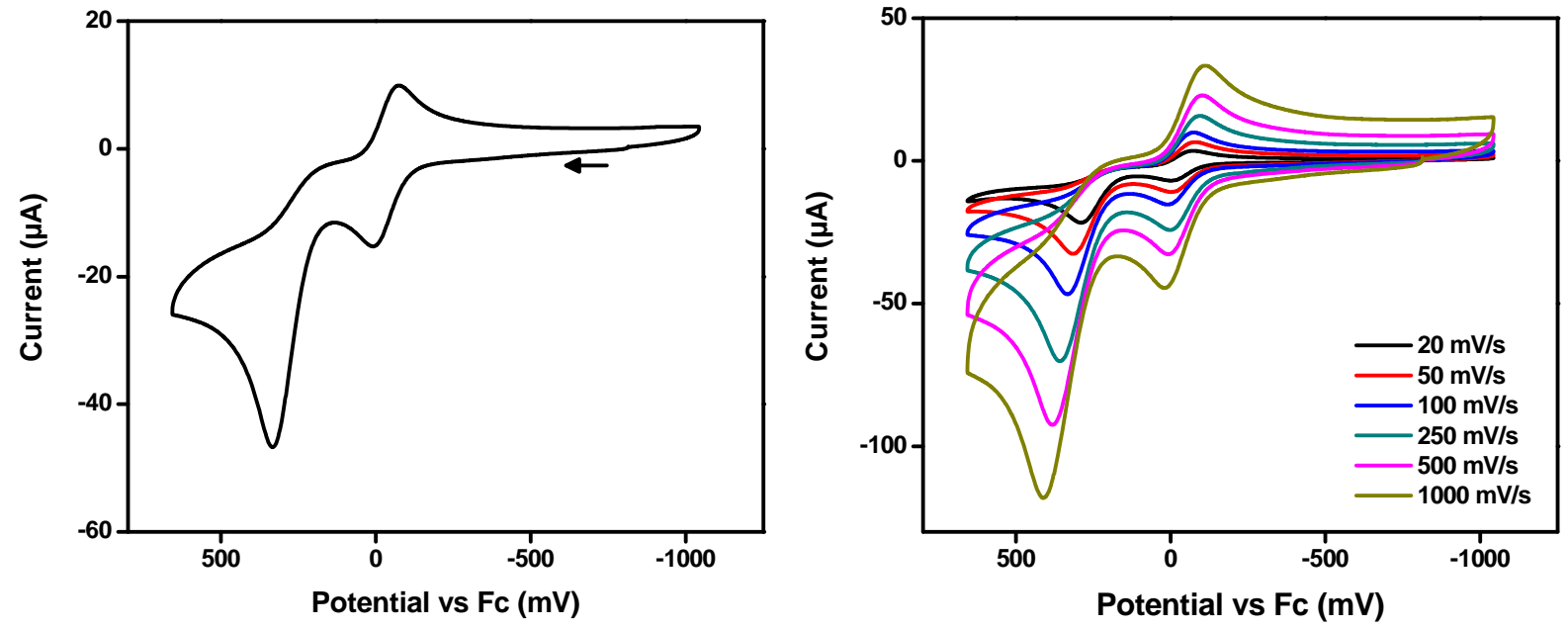

Figure S2: CVs of $\left[\left({ }^{\mathrm{Me}} \mathrm{N} 4\right) \mathrm{NiMe}_{2}\right] \mathrm{PF}_{6}$ in $\mathrm{MeCN}$ under $\mathrm{N}_{2}$ at $\mathrm{RT}\left(\left[\mathrm{Bu}_{4} \mathrm{NPF}_{6}\right]=0.1 \mathrm{M}\right.$, glassy carbon disk electrode), at $100 \mathrm{mV} / \mathrm{s}$ scan rate (left) and variable scan rates (right). Potentials vs $\mathrm{Fc} / \mathrm{Fc}^{+}(\mathrm{V}): \mathrm{E}_{\mathrm{pa}}=0.01$ and $0.36 \mathrm{~V}, \mathrm{E}_{\mathrm{pc}}=-0.07$. 

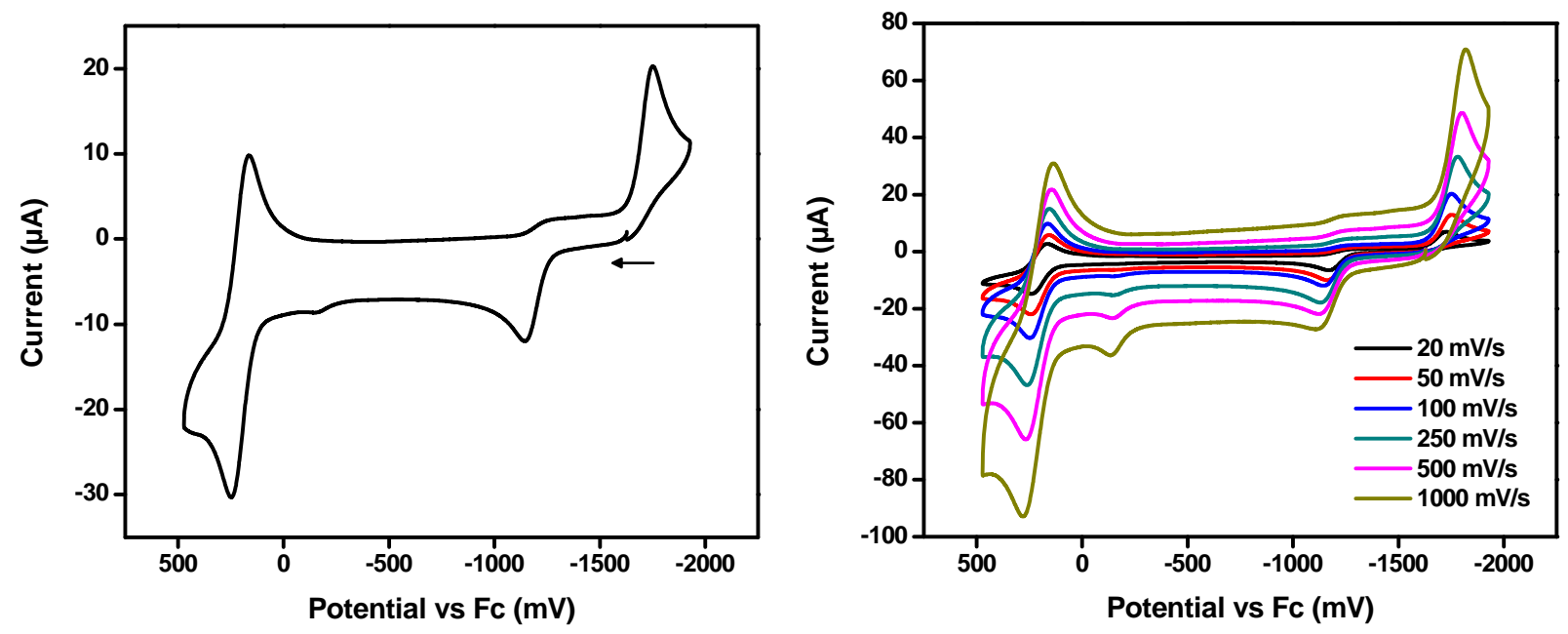

Figure S3: CVs of ( $\left.{ }^{\mathrm{Me}} \mathrm{N} 4\right) \mathrm{Ni}$ (cycloneophyl) in $\mathrm{MeCN}$ under $\mathrm{N}_{2}$ at $\mathrm{RT}\left(\left[\mathrm{Bu}_{4} \mathrm{NPF}_{6}\right]=0.1 \mathrm{M}\right.$, glassy carbon disk electrode), at $100 \mathrm{mV} / \mathrm{s}$ scan rate (left) and variable scan rates (right). Potentials vs $\mathrm{Fc} / \mathrm{Fc}^{+}(\mathrm{V}): \mathrm{E}_{\mathrm{pa}}=-1.17$ and $0.25, \mathrm{E}_{\mathrm{pc}}=0.17$ and -1.75 .
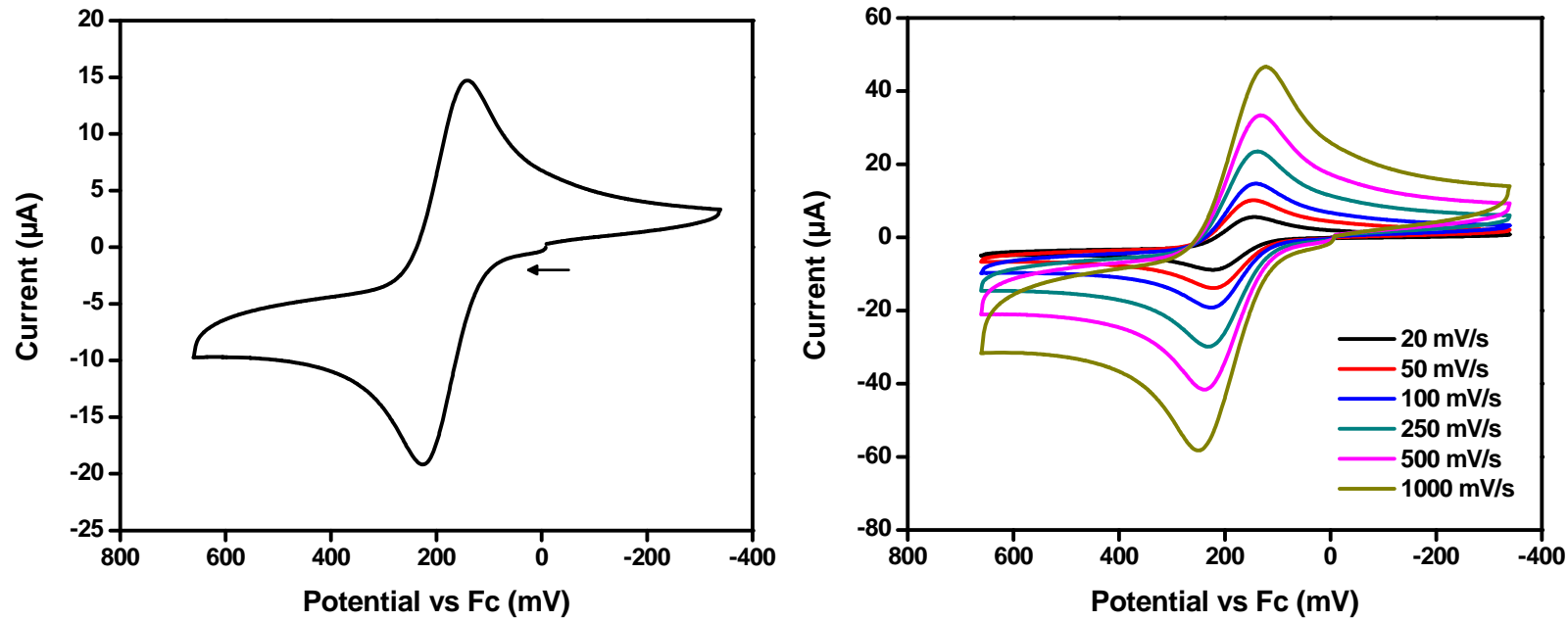

Figure S4: CVs of $\left[\left({ }^{\mathrm{Me}} \mathrm{N} 4\right) \mathrm{Ni}(\right.$ cycloneophyl $\left.)\right] \mathrm{PF}_{6}$ in $\mathrm{MeCN}$ under $\mathrm{N}_{2}$ at $\mathrm{RT}\left(\left[\mathrm{Bu}_{4} \mathrm{NPF}_{6}\right]=0.1 \mathrm{M}\right.$, glassy carbon disk electrode), at $100 \mathrm{mV} / \mathrm{s}$ scan rate (left) and variable scan rates (right). Potentials vs $\mathrm{Fc} / \mathrm{Fc}^{+}(\mathrm{V}): \mathrm{E}_{\mathrm{pa}}=0.25, \mathrm{E}_{\mathrm{pc}}=0.17$. 
IV. UV-Vis spectra of Isolated Ni Complexes
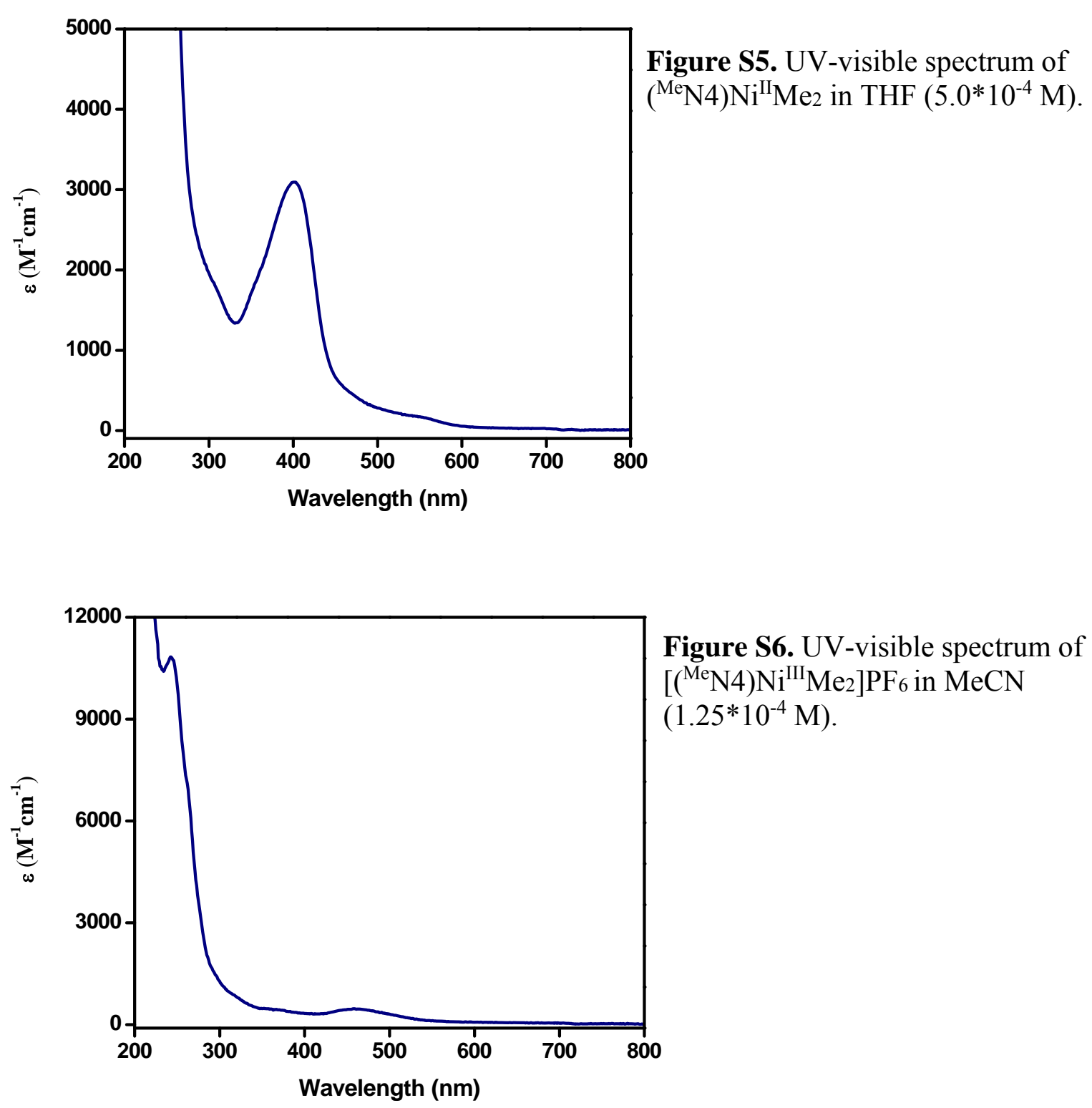

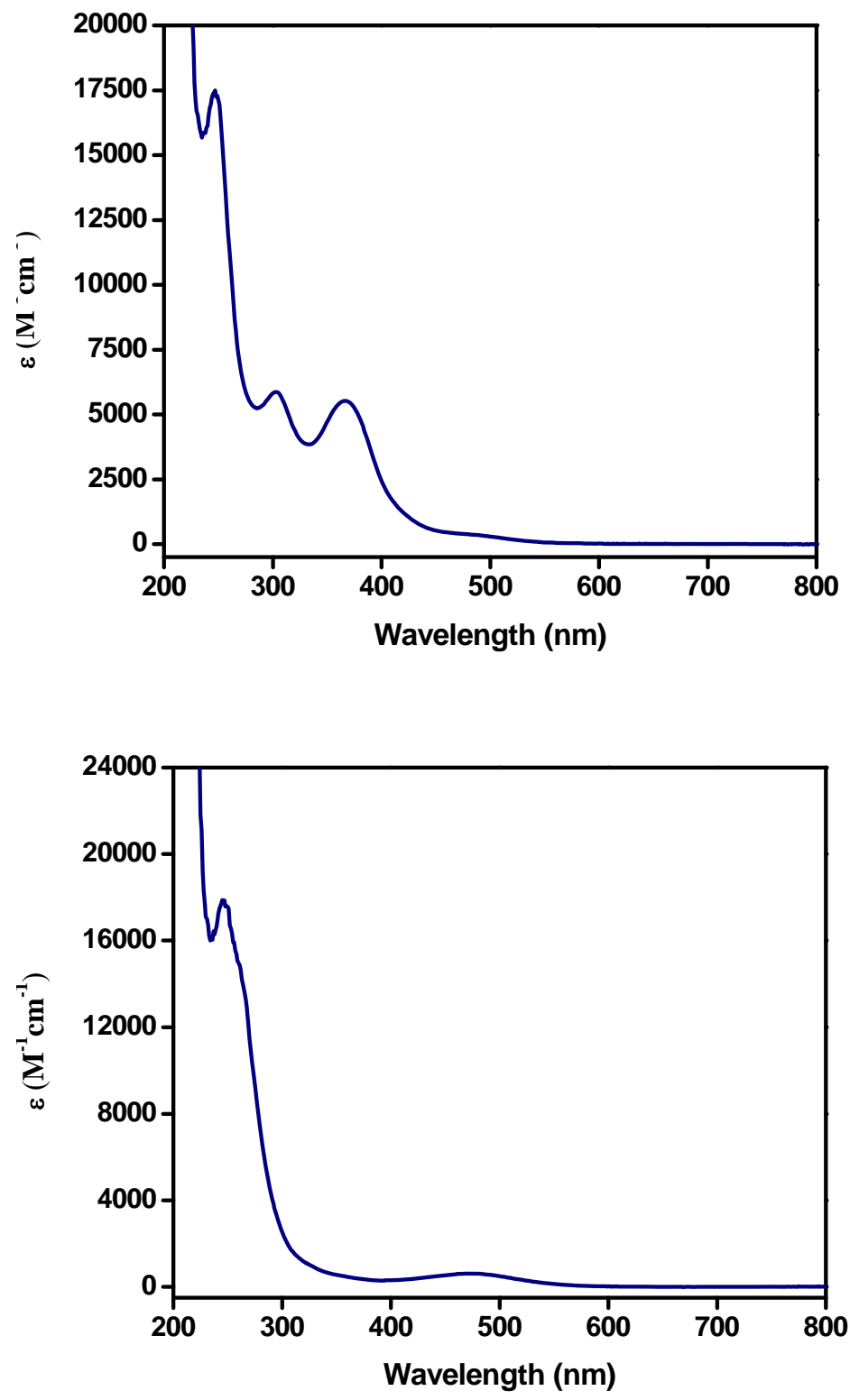

Figure S7. UV-visible spectrum of ( $\left.{ }^{\mathrm{Me}} \mathrm{N} 4\right) \mathrm{Ni}^{\mathrm{II}}$ (cycloneophyl) in $\mathrm{MeCN}$ $\left(1.25^{*} 10^{-4} \mathrm{M}\right)$.

Figure S8. UV-visible spectrum of $\left[\left({ }^{\mathrm{Me}} \mathrm{N} 4\right) \mathrm{Ni}^{\mathrm{III}}\right.$ (cycloneophyl)] $\mathrm{PF}_{6}$ in $\mathrm{MeCN}\left(1.25^{*} 10^{-4} \mathrm{M}\right)$. 


\section{Simulation of EPR spectra of Isolated $\mathrm{Ni}^{\mathrm{III}}$ Complexes}
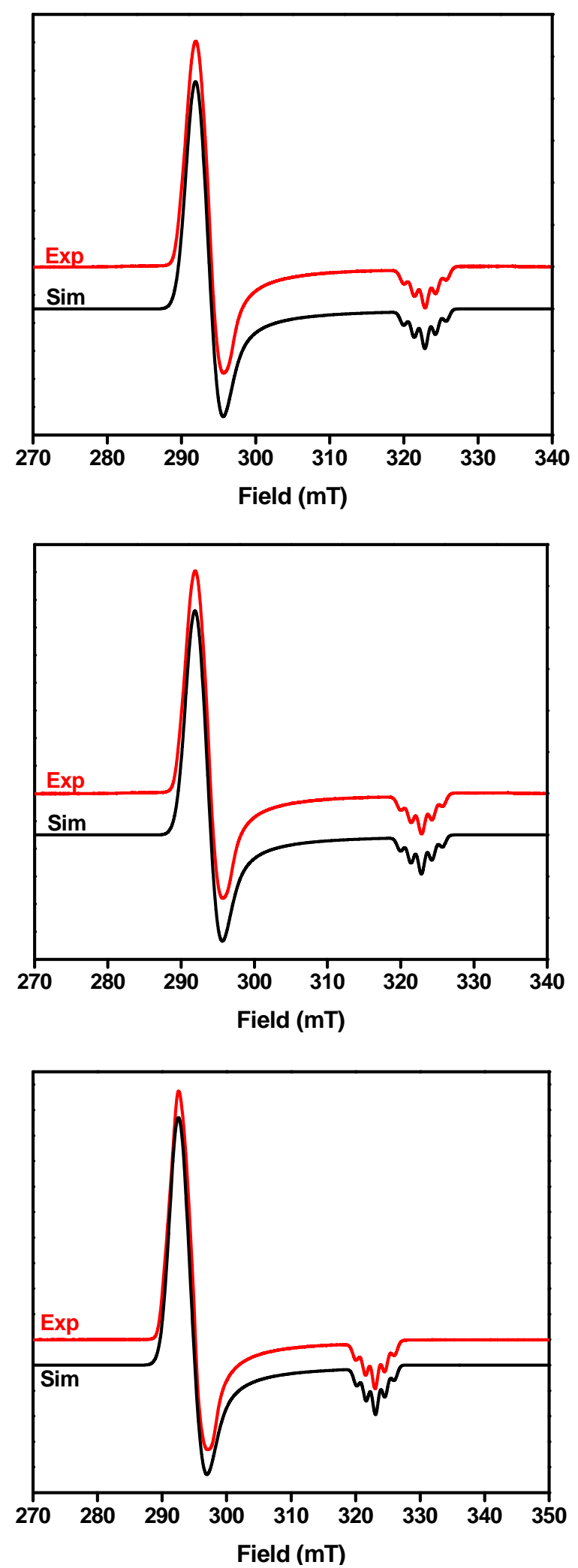

Figure S9. Experimental and simulated EPR spectra of isolated $\left[\left({ }^{\mathrm{Me}} \mathrm{N} 4\right) \mathrm{Ni}^{\mathrm{III}} \mathrm{Me}_{2}\right] \mathrm{PF}_{6}$ in frozen $\operatorname{PrCN}(77 \mathrm{~K})$.

Experimental conditions: frequency $\approx 9.097$ $\mathrm{GHz}$, power $=1 \mathrm{~mW}$, modulation frequency $=100 \mathrm{kHz}$, modulation amplitude $=3 \mathrm{G}$, time constant $=0.3 \mathrm{~s}$.

Simulation parameters: $\mathrm{g}_{\mathrm{x}}=2.2283, \mathrm{~g}_{\mathrm{y}}=$ $2.2100, \mathrm{~g}_{\mathrm{z}}=2.0137\left(\mathrm{~A}_{\mathrm{N}}=14.30 \mathrm{G}\right)$.

Figure S10. Experimental and simulated EPR spectra of isolated $\left[\left({ }^{\mathrm{Me}} \mathrm{N} 4\right) \mathrm{Ni}^{\mathrm{III}} \mathrm{Me}_{2}\right] \mathrm{BPh}_{4}$ in frozen $\mathrm{PrCN}(77$ $\mathrm{K})$.

Experimental conditions: frequency $\approx 9.097$ $\mathrm{GHz}$, power $=1 \mathrm{~mW}$, modulation frequency $=100 \mathrm{kHz}$, modulation amplitude $=3 \mathrm{G}$, time constant $=0.3 \mathrm{~s}$.

Simulation parameters: $\mathrm{g}_{\mathrm{x}}=2.2283, \mathrm{~g}_{\mathrm{y}}=$ $2.2100, \mathrm{~g}_{\mathrm{z}}=2.0137\left(\mathrm{~A}_{\mathrm{N}}=14.30 \mathrm{G}\right)$.

Figure S11. Experimental and simulated EPR spectra of $\left[\left({ }^{\mathrm{Me}} \mathrm{N} 4\right) \mathrm{Ni}^{\mathrm{III}}\right.$ (cycloneophyl) $] \mathrm{PF}_{6}$ in frozen PrCN (77 K).

Experimental conditions: frequency $\approx 9.097$ $\mathrm{GHz}$, power $=1 \mathrm{~mW}$, modulation frequency $=100 \mathrm{kHz}$, modulation amplitude $=3 \mathrm{G}$, time constant $=0.3 \mathrm{~s}$.

Simulation parameters: $\mathrm{g}_{\mathrm{x}}=2.2225, \mathrm{~g}_{\mathrm{y}}=$ $2.2012, \mathrm{~g}_{\mathrm{z}}=2.0116\left(\mathrm{~A}_{\mathrm{N}}=14.50 \mathrm{G}\right)$. 


\section{NMR Spectra of $\mathrm{Ni}^{\mathrm{IV}}$ Complex [( $\left.{ }^{\mathrm{Me}} \mathrm{N} 4\right) \mathrm{Ni}^{\mathrm{IV}}$ (cycloneophyl)](PF $)_{2}$}

A d3-MeCN solution of [( $\left.{ }^{\mathrm{Me}} \mathrm{N} 4\right) \mathrm{Ni}^{\mathrm{III}}$ (cycloneophyl) $] \mathrm{PF}_{6}(20.0 \mathrm{mg}, 0.033 \mathrm{mmol})$ and NOPF6 $(29.0$ $\mathrm{mg}, 0.166 \mathrm{mmol}$ ) was stirred at $-40^{\circ} \mathrm{C}$ for 5 minutes. The resulting solution was then transferred to a pre-cooled NMR tube at $-40^{\circ} \mathrm{C}$. The NMR tube was then sealed and quickly removed from the glove box and placed in the NMR, which the temperature control was set at $-15^{\circ} \mathrm{C}$ for all NMR experiments.

${ }^{1} \mathrm{H}-\mathrm{NMR}(300 \mathrm{MHz}, \mathrm{CDCl} 3), \delta(\mathrm{ppm}): 8.05$ (t, 1H, F), $7.97(\mathrm{t}, 1 \mathrm{H}, \mathbf{G}), 7.51(\mathrm{~d}, 2 \mathrm{H}, \mathbf{I}), 7.41$ (d, 2H, H), 7.25 (t, 1H, L), 7.19 (d, 1H, K), 6.92 (t, 1H, M), 6.55 (d, 1H, N), 5.33 (s, 2H, O), 5.01 (d, 2H, E), 4.56 (d, 2H, B), 4.22 (d, 2H, D), 4.08 (d, 2H, C), 2.38 (s, 6H, A), 1.67 (s, 6H, J).

${ }^{13} \mathrm{C}-\mathrm{NMR}(600 \mathrm{MHz}, \mathrm{CDCl} 3), \delta$ (ppm): 159.42 (p, k), 158.44 (i), 156.25 (g), 154.50 (c), 144.89 (e), 133.85 (o), 132.31 (n), 131.31 (l), 131.15 (m), 125.82 (d), 125.07 (f), 79.77 (h), 77.38 (b), $57.31(\mathbf{a}), 36.00(\mathbf{j})$.
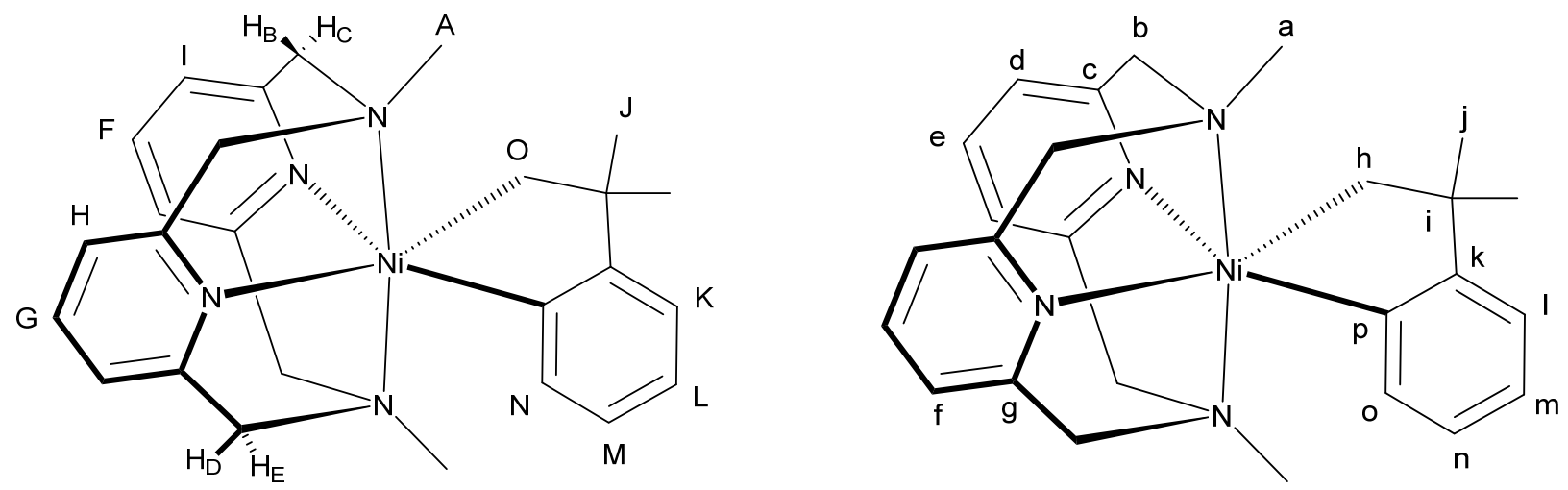

Figure S12. Proton (left) and carbon (right) structural assignments from NMR experiments. 


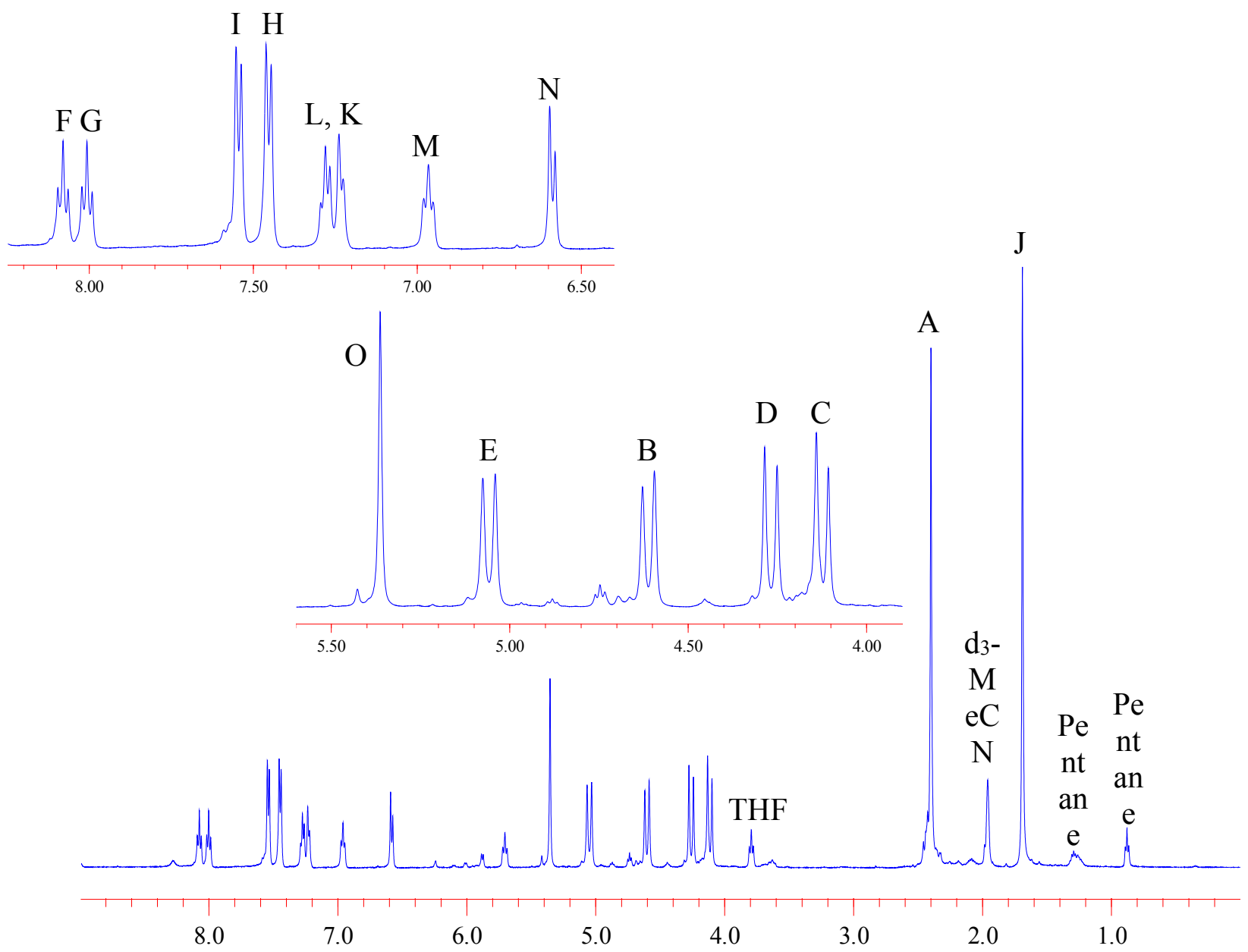

Figure S13. ${ }^{1} \mathrm{H}$ NMR spectrum of $\left[\left({ }^{\mathrm{Me}} \mathrm{N} 4\right) \mathrm{Ni}^{\mathrm{IV}}(\right.$ cycloneophyl $\left.)\right](\mathrm{PF} 6)_{2}$ in $\mathrm{d}_{3}-\mathrm{MeCN}$ at $-15^{\circ} \mathrm{C}$. 


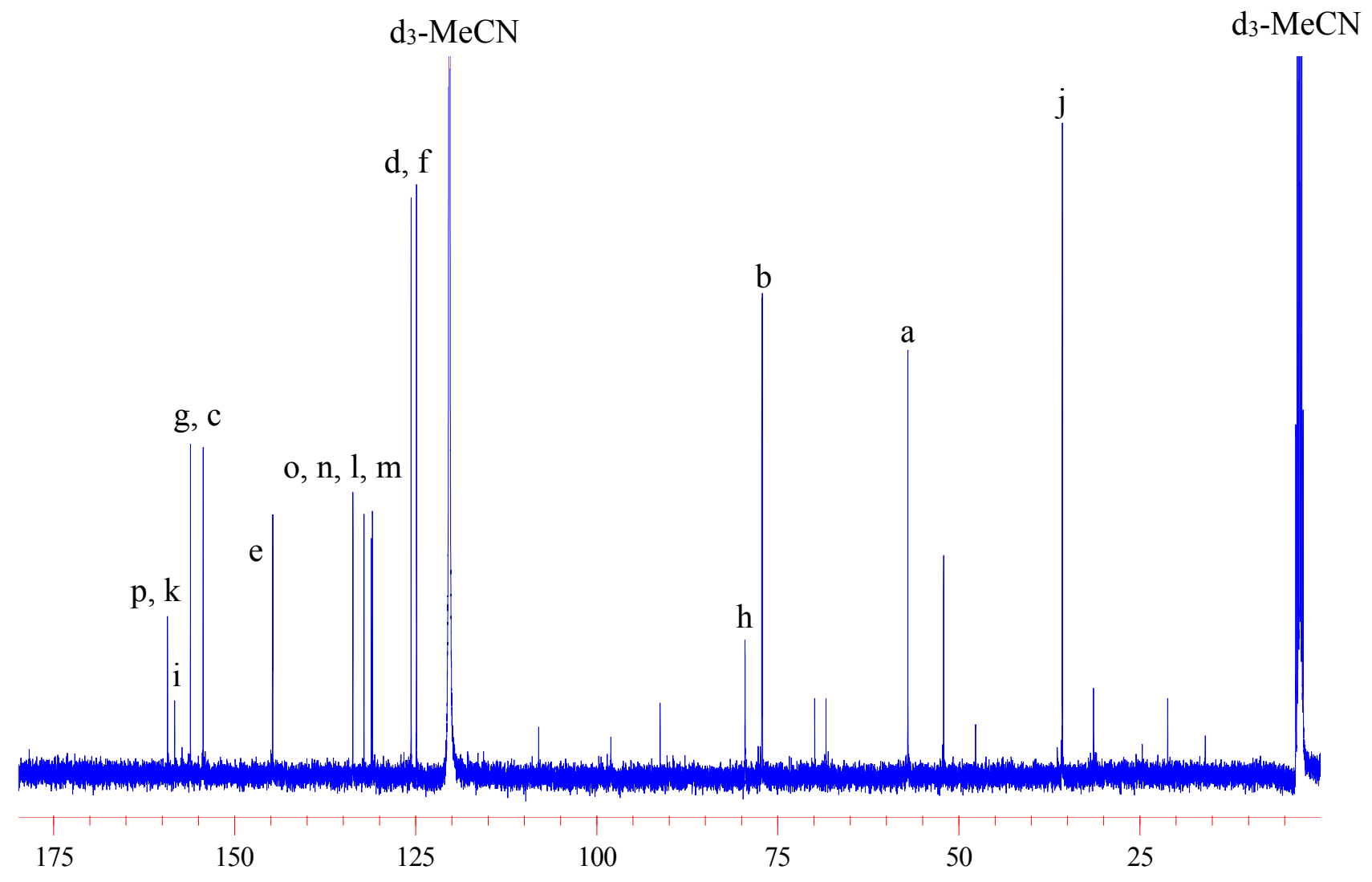

Figure S14. ${ }^{13} \mathrm{C}$ NMR spectrum of $\left[\left({ }^{\mathrm{Me}} \mathrm{N} 4\right) \mathrm{Ni}^{\mathrm{IV}}\right.$ (cycloneophyl) $]\left(\mathrm{PF}_{6}\right)_{2}$ in $\mathrm{d}_{3}-\mathrm{MeCN}$ at $-15^{\circ} \mathrm{C}$. 


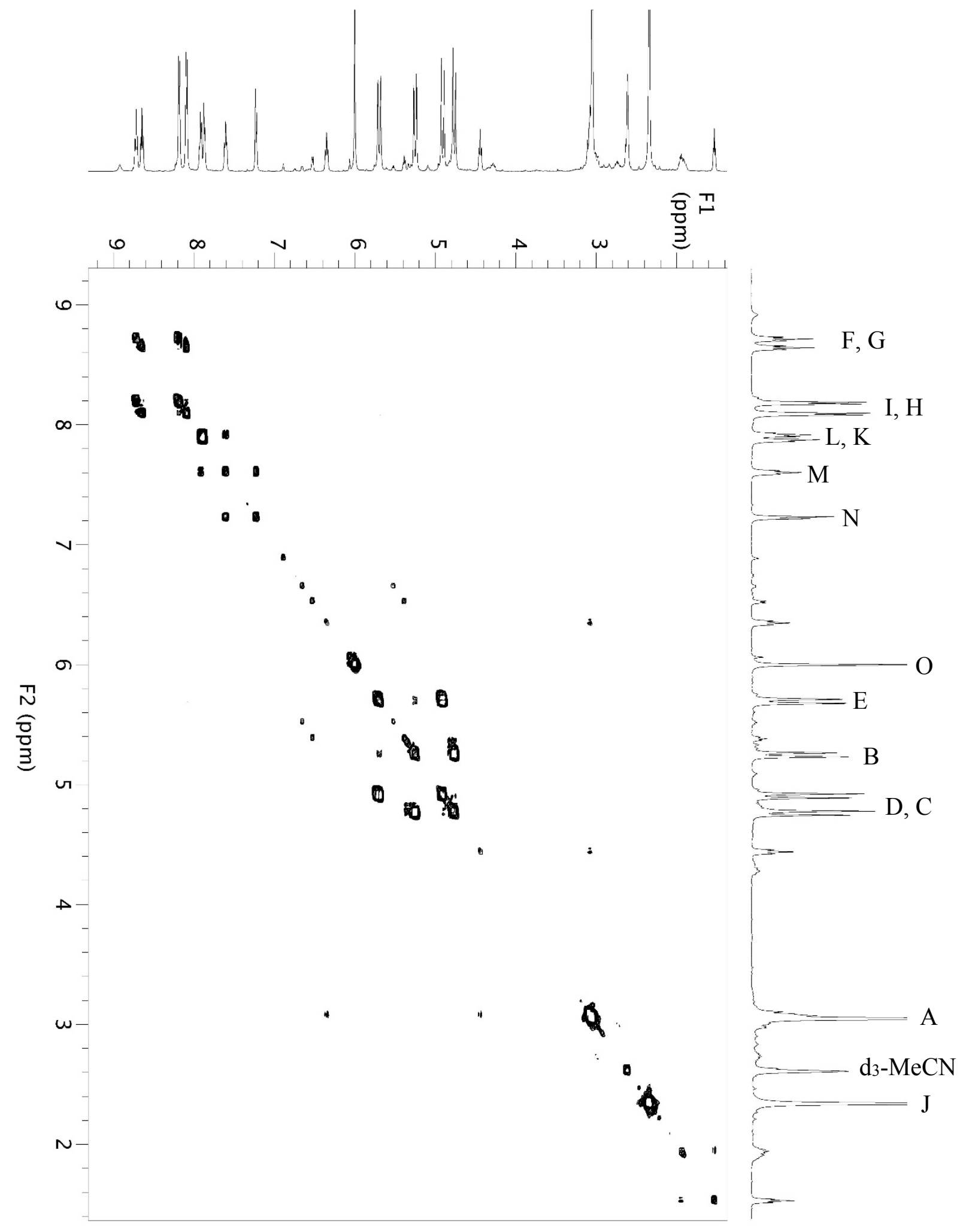

Figure S15. ${ }^{1} \mathrm{H}-{ }^{1} \mathrm{H}$ COSY spectrum of $\left[\left({ }^{\mathrm{Me}} \mathrm{N} 4\right) \mathrm{Ni}^{\mathrm{IV}}\right.$ (cycloneophyl) $]\left(\mathrm{PF}_{6}\right)_{2}$ in $\mathrm{d}_{3}-\mathrm{MeCN}$ at $-15^{\circ} \mathrm{C}$. 


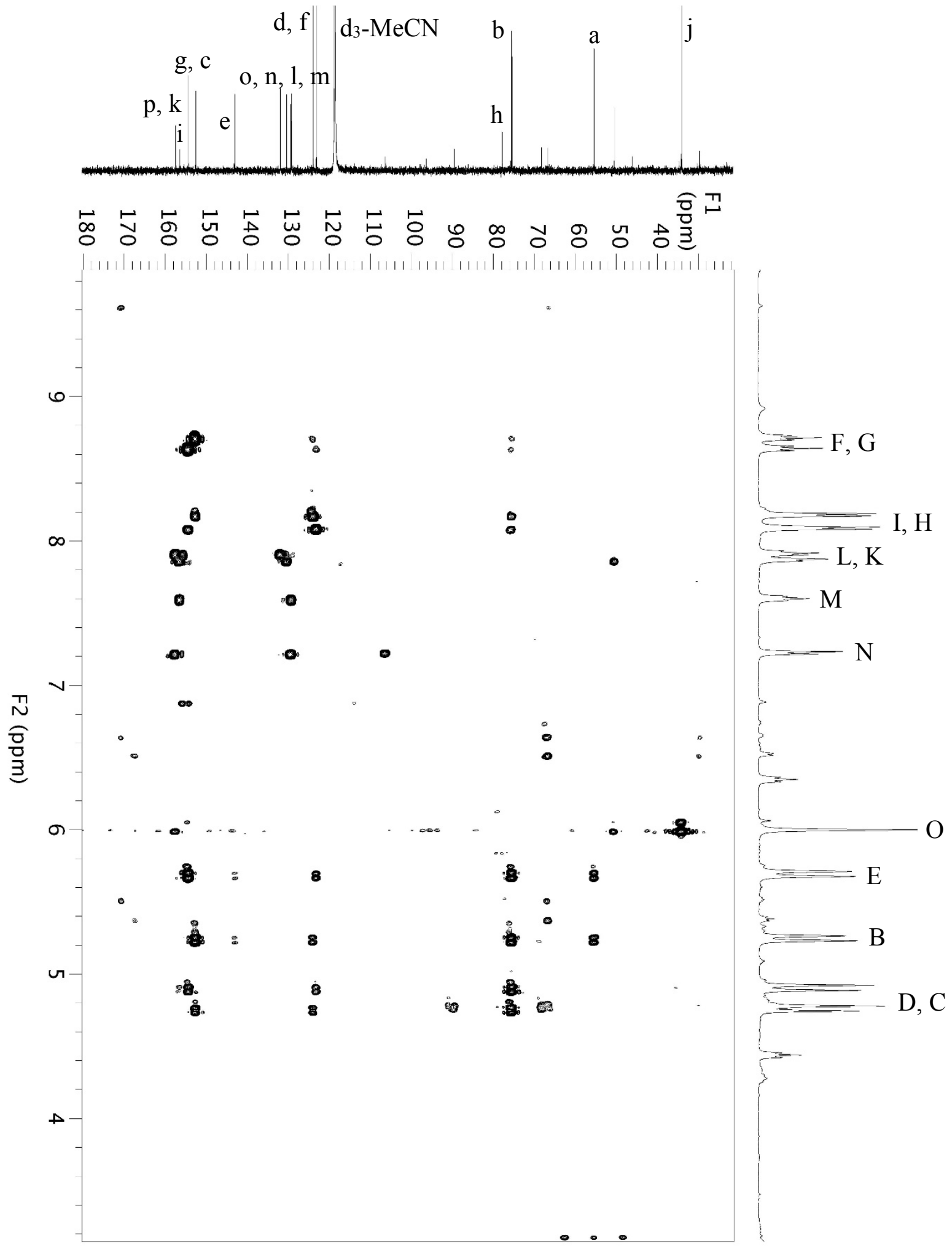

Figure S16. ${ }^{1} \mathrm{H}-{ }^{13} \mathrm{C}$ HMBC spectrum of $\left[\left({ }^{\mathrm{Me}} \mathrm{N} 4\right) \mathrm{Ni}^{\mathrm{IV}}\right.$ (cycloneophyl) $]\left(\mathrm{PF}_{6}\right) 2$ in $\mathrm{d}_{3}-\mathrm{MeCN}$ at $-15^{\circ} \mathrm{C}$. 


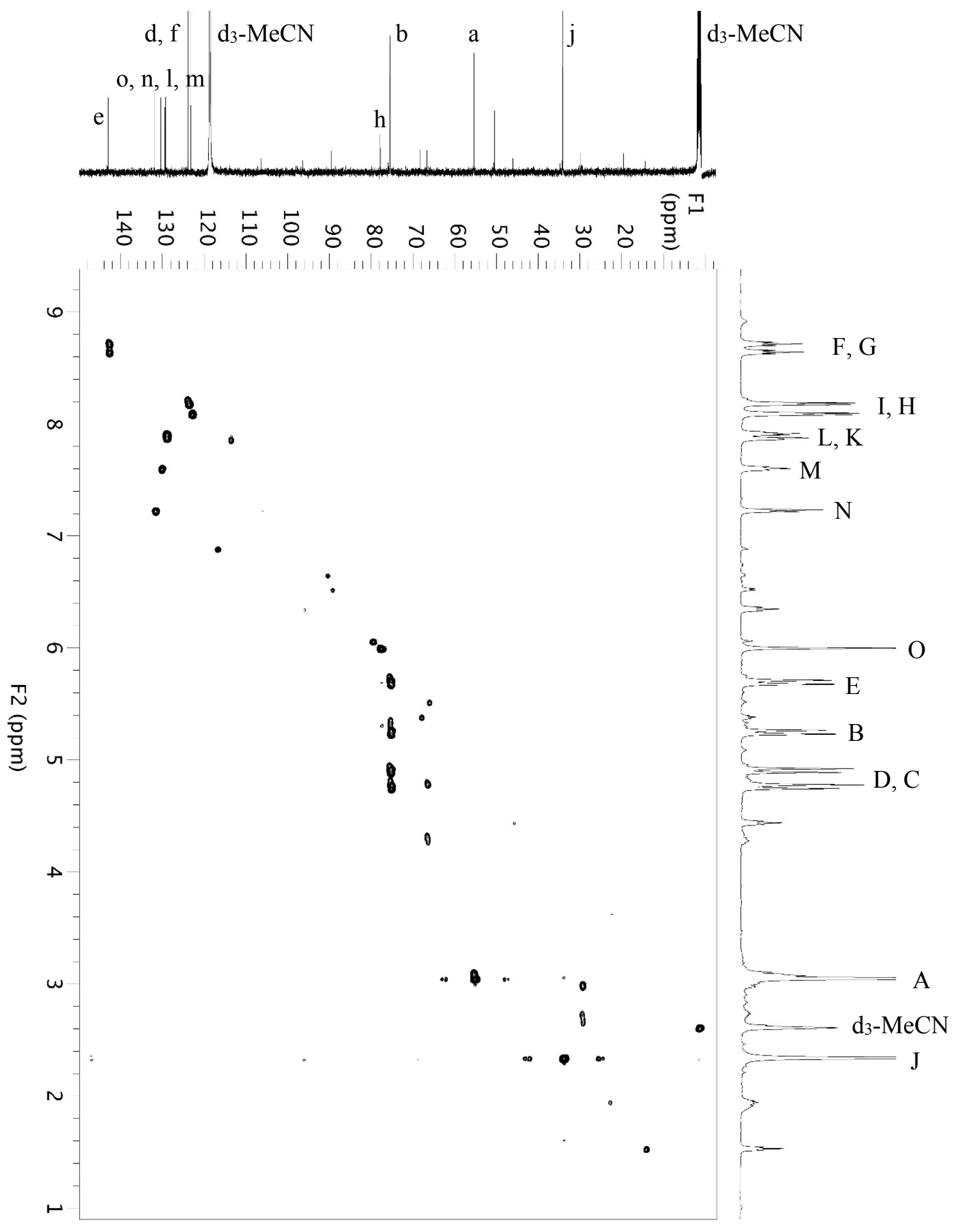

Figure S17. ${ }^{1} \mathrm{H}_{-}{ }^{13} \mathrm{C}$ HSQC spectrum of $\left[\left({ }^{\mathrm{Me}} \mathrm{N} 4\right) \mathrm{Ni}^{\mathrm{IV}}\right.$ (cycloneophyl) $]\left(\mathrm{PF}_{6}\right)_{2}$ in $\mathrm{d}_{3}-\mathrm{MeCN}$ at $-15^{\circ} \mathrm{C}$. 


\section{XPS of Isolated Ni Complexes}

Solid samples of $\left[\left({ }^{\mathrm{Me}} \mathrm{N} 4\right) \mathrm{Ni}^{\mathrm{III}}\right.$ (cycloneophyl) $] \mathrm{PF}_{6}$ and $\left[\left({ }^{\mathrm{Me}} \mathrm{N} 4\right) \mathrm{Ni}^{\mathrm{III}}\right.$ (cycloneophyl) $]\left(\mathrm{PF}_{6}\right)_{2}$ were prepared and stored on dry ice prior to XPS analysis. Immediately prior to analysis, the two samples were transferred at RT to a piece of tape pre-fixed to the XPS plate. The samples were compressed and quickly loaded into the instrument. XPS spectra were recorded on a PHI 5000 Versa Probe II X-Ray Photoelectron Spectrometer. The XPS experiments was performed in conjunction with the Washington University Institute of Materials Science and Engineering.

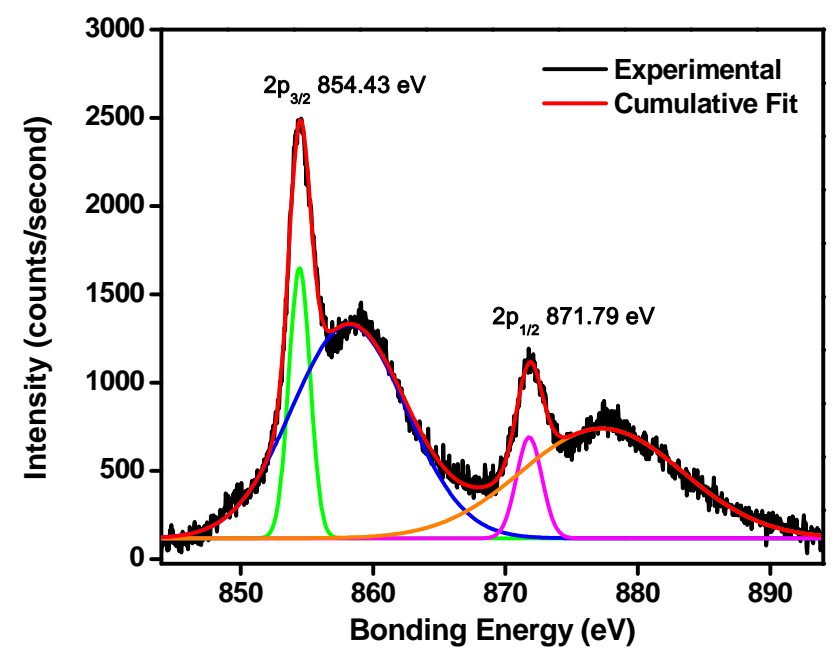

Figure S18. XPS spectrum of [( $\left.{ }^{\mathrm{Me}} \mathrm{N} 4\right) \mathrm{Ni}^{\mathrm{III}}$ (cycloneophyl)]PF 6 , Bonding energies: ${ }_{p^{3 / 2}}, 854.43$ $\mathrm{eV}$ and $2_{p^{1 / 2}}, 871.79 \mathrm{eV}$.

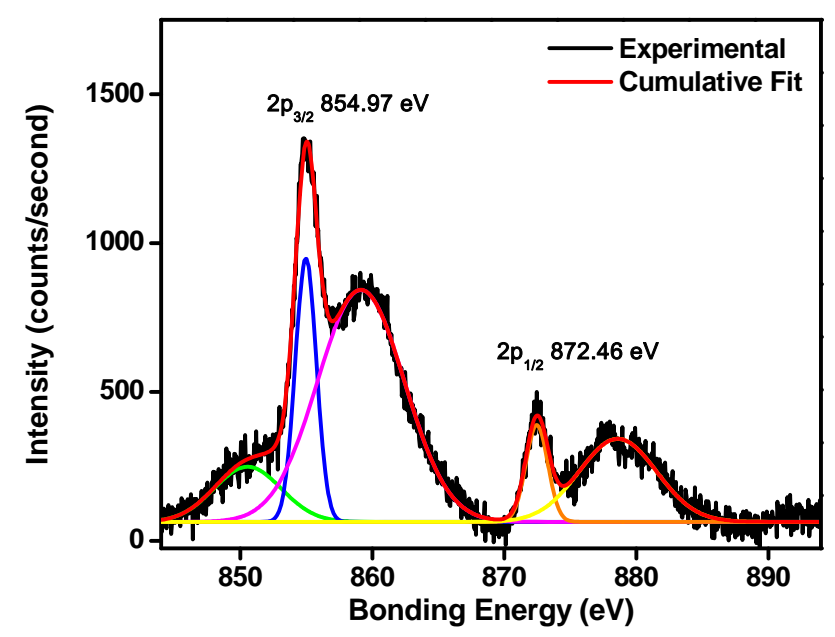

Figure S19. XPS spectrum of $\left[\left({ }^{\mathrm{Me}} \mathrm{N} 4\right) \mathrm{Ni}^{\mathrm{IV}}\right.$ (cycloneophyl) $]\left(\mathrm{PF}_{6}\right) 2$, Bonding energies: $2 p^{3 / 2}$, $854.97 \mathrm{eV}$ and $2_{p^{1 / 2}}, 872.46 \mathrm{eV}$. 


\section{Computational Details}

The density functional theory (DFT) and time-dependent density functional theory (TD-DFT) calculations were performed with the program package Gaussian 09. ${ }^{6}$ The UB3LYP hybrid functional with the $6-31 \mathrm{G}^{*}$ basis set for $\mathrm{C}, \mathrm{N}, \mathrm{H}$ and the modified $\mathrm{m6}-31 \mathrm{G} *$ basis set for Ni were employed. This combination of hybrid functional and basis sets have been previously shown to work well for reproducing experimental parameters of $\mathrm{Ni}$ complexes. ${ }^{7-8}$ Geometry optimization calculations were performed using the X-ray coordinates as the starting geometry. The ground state wave function was investigated by analyzing the frontier MOs and spin density plot using GaussView 5.0, and the atomic contributions to MOs and spin density plot were calculated using the program Chemissian. ${ }^{9}$

\begin{tabular}{cccccc}
\multicolumn{6}{c}{ Optimized Geometry Cartesian coordinates for $\left[\left({ }^{\mathrm{Me}} \mathrm{N} 4\right) \mathrm{Ni}{ }^{\mathrm{III}} \mathrm{M}\right.$} \\
1 & 28 & 0 & 0.000000 & 0.000000 & 0.000000 \\
2 & 7 & 0 & 2.021783 & 0.000000 & 0.000000 \\
3 & 7 & 0 & 0.247552 & 2.005717 & 0.000000 \\
4 & 7 & 0 & 0.413965 & 0.365410 & -2.235960 \\
5 & 7 & 0 & 0.419656 & 0.372352 & 2.241174 \\
6 & 6 & 0 & -1.956410 & 0.053589 & -0.035459 \\
7 & 1 & 0 & -2.258512 & 1.055664 & -0.366127 \\
8 & 1 & 0 & -2.356453 & -0.119554 & 0.969826 \\
9 & 1 & 0 & -2.393318 & -0.686062 & -0.713631 \\
10 & 6 & 0 & -0.201475 & -1.944472 & 0.044929 \\
11 & 1 & 0 & -0.454668 & -2.324964 & -0.950937 \\
12 & 1 & 0 & -0.973494 & -2.274171 & 0.747328 \\
13 & 1 & 0 & 0.760566 & -2.375593 & 0.350811 \\
14 & 6 & 0 & 2.676229 & 0.180104 & 1.158779 \\
15 & 6 & 0 & 4.048366 & 0.433168 & 1.178537 \\
16 & 1 & 0 & 4.559644 & 0.577033 & 2.124988 \\
17 & 6 & 0 & 4.733239 & 0.512992 & -0.033264 \\
18 & 1 & 0 & 5.800032 & 0.716090 & -0.045749 \\
19 & 6 & 0 & 4.033088 & 0.360188 & -1.230792 \\
20 & 1 & 0 & 4.532767 & 0.447066 & -2.190100 \\
21 & 6 & 0 & 2.662779 & 0.109540 & -1.177199 \\
22 & 6 & 0 & 1.813146 & -0.096371 & -2.410499 \\
23 & 1 & 0 & 2.286108 & 0.377490 & -3.281510 \\
24 & 1 & 0 & 1.774224 & -1.173246 & -2.610986 \\
25 & 6 & 0 & 0.263782 & 1.830099 & -2.422684 \\
26 & 1 & 0 & -0.769303 & 2.012976 & -2.738953 \\
27 & 1 & 0 & 0.911701 & 2.199071 & -3.229554 \\
28 & 6 & 0 & 0.498608 & 2.631796 & -1.161196 \\
29 & 6 & 0 & 0.905807 & 3.966306 & -1.185290
\end{tabular}




$\begin{array}{rrrrrr}30 & 1 & 0 & 1.106378 & 4.455262 & -2.133162 \\ 31 & 6 & 0 & 1.061681 & 4.641538 & 0.024707 \\ 32 & 1 & 0 & 1.382846 & 5.678954 & 0.034046 \\ 33 & 6 & 0 & 0.829655 & 3.967515 & 1.224369 \\ 34 & 1 & 0 & 0.970303 & 4.458019 & 2.182079 \\ 35 & 6 & 0 & 0.427851 & 2.633527 & 1.175489 \\ 36 & 6 & 0 & 0.129329 & 1.816944 & 2.412688 \\ 37 & 1 & 0 & 0.660739 & 2.232452 & 3.279775 \\ 38 & 1 & 0 & -0.943155 & 1.906679 & 2.620101 \\ 39 & 6 & 0 & 1.856366 & 0.045967 & 2.423202 \\ 40 & 1 & 0 & 1.914456 & -1.000445 & 2.742975 \\ 41 & 1 & 0 & 2.304417 & 0.646917 & 3.226474 \\ 42 & 6 & 0 & -0.462843 & -0.344124 & -3.189511 \\ 43 & 1 & 0 & -0.380189 & -1.421117 & -3.028344 \\ 44 & 1 & 0 & -0.193209 & -0.122845 & -4.233179 \\ 45 & 1 & 0 & -1.498829 & -0.041796 & -3.022013 \\ 46 & 6 & 0 & -0.387421 & -0.411066 & 3.197862 \\ 47 & 1 & 0 & -1.447138 & -0.199663 & 3.039544 \\ 48 & 1 & 0 & -0.132352 & -0.168948 & 4.240543 \\ 49 & 1 & 0 & -0.213143 & -1.476272 & 3.031335\end{array}$




\section{Reactivity of Ni Complexes}

General procedure for the reactivity studies of ( $\left.{ }^{\mathrm{Me}} \mathrm{N} 4\right) \mathrm{Ni}$ complexes. In $\mathrm{N}_{2}$-filled glove box, a solution of $5-7 \mathrm{mg}$ of $\left({ }^{\mathrm{Me}} \mathrm{N} 4\right) \mathrm{Ni}$ complex in $2.0 \mathrm{~mL}$ of $\mathrm{d}_{3}-\mathrm{MeCN}$ was added into a NMR tube containing 1,3,5-trimethoxybenzene as an internal standard. To this solution additional reagents $\left[\mathrm{FcPF}_{6},{ }^{\mathrm{Ac}} \mathrm{FcBF}_{4}, \mathrm{H}_{2} \mathrm{O}_{2}, \mathrm{O}_{2}, \mathrm{PhI}\left(\mathrm{Py}^{\mathrm{OMe}}\right)_{2}(\mathrm{OTf})_{2}, \mathrm{CD}_{3} \mathrm{I}, \mathrm{PhMgBr}, \mathrm{MeMgCl}\right.$, or TEMPO] were added in $\mathrm{d}_{3}-\mathrm{MeCN}$. For crossover experiments, equimolar amounts of $\left({ }^{\mathrm{Me}} \mathrm{N} 4\right) \mathrm{NiMe}_{2}$ and $\left({ }^{\mathrm{Me}} \mathrm{N} 4\right) \mathrm{Ni}\left(\mathrm{CD}_{3}\right)_{2}$ in $\mathrm{d}_{3}-\mathrm{MeCN}$ were added to the $\mathrm{NMR}$ tube as separate solutions. For all experiments the NMR tube was filled to the top with additional $\mathrm{d}_{3}-\mathrm{MeCN}$ (so that no headspace was left to avoid the escape of volatiles), sealed with a septum, and taken out of the glove box. The reaction mixtures were mixed carefully to form homogeneous solutions, and then kept in the dark and periodically monitored by ${ }^{1} \mathrm{H}$ NMR until no additional changes were observed. The average product yields from at least two independent experiments were determined by NMR integration using 1,3,5-trimethoxybenzene as an internal standard. For the reactivity of $\left({ }^{\mathrm{Me}} \mathrm{N} 4\right) \mathrm{Ni}^{\mathrm{III}}$ complexes, the amount of $\left[\left({ }^{\mathrm{Me}} \mathrm{N} 4\right) \mathrm{Ni}^{\mathrm{III}} \mathrm{Me}_{2}\right]^{+}$in solution was determined using EPR spin quantitation.

Ethane. ${ }^{1} \mathrm{H}$ NMR $\left(\mathrm{d}_{3}-\mathrm{MeCN}\right), \delta: 0.85$ (s). Ethane-d $3 .{ }^{1} \mathrm{H}$ NMR $\left(\mathrm{d}_{3}-\mathrm{MeCN}\right), \delta: 0.81$ (sept). Methane. ${ }^{1} \mathrm{H}$ NMR $\left(\mathrm{d}_{3}-\mathrm{MeCN}\right), \delta: 0.20$ (s). Methane- $\mathrm{d}_{1} .{ }^{1} \mathrm{H}$ NMR $\left(\mathrm{d}_{3}-\mathrm{MeCN}\right), \delta: 0.18(\mathrm{t})$. 1-Methoxy-2,2,6,6-tetramethylpiperidine (TEMPO-CH3). ${ }^{1} \mathrm{H}$ NMR ( $\left.\mathrm{d}_{3}-\mathrm{MeCN}\right), \delta: 1.05(\mathrm{~s}, 6 \mathrm{H})$, $1.14(\mathrm{~s}, 6 \mathrm{H}), 1.36-1.56(\mathrm{~m}, 6 \mathrm{H}), 3.56(\mathrm{~s}, 6 \mathrm{H})$.

1-Methoxy-d3-2,2,6,6-tetramethylpiperidine (TEMPO-CD 3$).{ }^{1} \mathrm{H}$ NMR $\left(\mathrm{d}_{3}-\mathrm{MeCN}\right), \delta: 1.07$ (s, $6 \mathrm{H}), 1.15(\mathrm{~s}, 6 \mathrm{H}), 1.25-1.50(\mathrm{~m}, 6 \mathrm{H})$.

1-Hydroxy-2,2,6,6-tetramethylpiperidine (TEMPO-H). ${ }^{1} \mathrm{H}$ NMR (d $\left.3-\mathrm{MeCN}\right), \delta: 1.06(\mathrm{~s}, 12 \mathrm{H})$, $1.45(\mathrm{~s}, 6 \mathrm{H})$.

GC Product Analysis. After no changes were observed by NMR, the reaction mixtures were quenched with $100 \mu \mathrm{L}$ of $1.0 \mathrm{M} \mathrm{HCl}$ and $5 \mathrm{~mL} \mathrm{H} \mathrm{H}_{2} \mathrm{O}$, and extracted with ethyl ether $(6 \mathrm{~mL})$. The organic layer was then separated and dried over $\mathrm{MgSO}_{4}$. The yield of product(s) was obtained by GC/FID using 1,3,5-trimethoxybenzene as the internal standard. The identity of the products were confirmed by GC-MS.

Note: Performing the reductive elimination at elevated temperatures does lead to a faster reductive elimination of ethane, yet appreciable amounts of methane were also observed. Thus, all reactivity studies were performed at room temperature to limit the side reactions. 


\section{A. Reactivity of ( $\left.{ }^{\mathrm{Me}} \mathrm{N} 4\right) \mathrm{Ni}(\mathrm{II}) \mathrm{Me}_{2}$.}

Table S1. Yields of the products of elimination from $\left({ }^{\mathrm{Me}} \mathrm{N} 4\right) \mathrm{NiMe}_{2}$ in $\mathrm{d}_{3}-\mathrm{MeCN}$ at RT.

Note: Formation of Ni black was also detected at the end of the reaction, suggesting a different reaction mechanism under these conditions.

\begin{tabular}{|c|c|c|}
\hline Time, h & Me-Me, $\%$ & Me-H/D, $\%$ \\
\hline 1 & $3 \pm 1$ & $0 \pm 1$ \\
\hline 2 & $5 \pm 2$ & $2 \pm 1$ \\
\hline 4 & $8 \pm 1$ & $3 \pm 1$ \\
\hline 8 & $11 \pm 1$ & $5 \pm 1$ \\
\hline
\end{tabular}

\section{B. Reactivity of ( $\left.{ }^{\mathrm{Me}} \mathrm{N} 4\right) \mathrm{Ni}(\mathrm{II}) \mathrm{Me}_{2}$ with 1 equivalent $\mathrm{FCPF}_{6}$.}

Table S2. Yields of the products from the reaction of $\left({ }^{\mathrm{Me}} \mathrm{N} 4\right) \mathrm{NiMe}_{2}$ with 1 equivalent of $\mathrm{FcPF}_{6}$ in $\mathrm{d}_{3}-\mathrm{MeCN}$ at $\mathrm{RT}$.

\begin{tabular}{|c|c|c|}
\hline Time, $\mathbf{h}$ & Me-Me, $\%$ & Me-H/D, $\%$ \\
\hline 1 & $31 \pm 3$ & $5 \pm 2$ \\
\hline 2 & $36 \pm 3$ & $6 \pm 2$ \\
\hline 4 & $44 \pm 4$ & $10 \pm 2$ \\
\hline 8 & $61 \pm 3$ & $24 \pm 2$ \\
\hline
\end{tabular}

\section{Reactivity of ( $\left.{ }^{\mathrm{Me}} \mathrm{N} 4\right) \mathrm{Ni}(\mathrm{II}) \mathrm{Me}_{2}$ with 1 equivalent ${ }^{{ }^{\mathrm{c}} \mathrm{FcPF}_{6} \text {. }}$}

Table S3. Yields of the products from the reaction of ( $\left.{ }^{\mathrm{Me}} \mathrm{N} 4\right) \mathrm{NiMe}_{2}$ with 1 equivalent of ${ }^{\mathrm{Ac}} \mathrm{FcPF}_{6}$ in $\mathrm{d}_{3}-\mathrm{MeCN}$ at $\mathrm{RT}$.

\begin{tabular}{|c|c|c|}
\hline Time, $\mathbf{h}$ & Me-Me, $\%$ & Me-H/D, $\%$ \\
\hline 1 & $35 \pm 1$ & $5 \pm 1$ \\
\hline 2 & $37 \pm 2$ & $5 \pm 1$ \\
\hline 4 & $43 \pm 2$ & $11 \pm 2$ \\
\hline 8 & $62 \pm 3$ & $20 \pm 2$ \\
\hline
\end{tabular}

Table S4. Yields of the products from the reaction of ( $\left.{ }^{\mathrm{Me}} \mathrm{N} 4\right) \mathrm{NiMe}_{2}$ with 1 equivalent of ${ }^{\mathrm{Ac}} \mathrm{FcPF}_{6}$ and 2 equivalents of TEMPO in $\mathrm{d}_{3}-\mathrm{MeCN}$ at RT.

\begin{tabular}{|c|c|c|}
\hline Time, $\mathbf{h}$ & Me-Me, $\%$ & Me-H/D, $\%$ \\
\hline 1 & $37 \pm 2$ & $4 \pm 2$ \\
\hline 2 & $42 \pm 1$ & $4 \pm 2$ \\
\hline 4 & $53 \pm 3$ & $6 \pm 2$ \\
\hline 8 & $63 \pm 3$ & $11 \pm 2$ \\
\hline
\end{tabular}


Table S5. Yields of the products from the crossover of $\left({ }^{\mathrm{Me}} \mathrm{N} 4\right) \mathrm{NiMe}_{2}$ and $\left({ }^{\mathrm{Me}} \mathrm{N} 4\right) \mathrm{Ni}\left(\mathrm{CD}_{3}\right)_{2}$ with 1 equivalent of ${ }^{\mathrm{Ac}} \mathrm{FcPF}_{6}$ in $\mathrm{d}_{3}-\mathrm{MeCN}$ at $\mathrm{RT}$. The amount of $\mathrm{CD}_{3}-\mathrm{CD}_{3}$ was obtained by subtraction from the overall ethane yield for the non-deuteraed ( $\left.{ }^{\mathrm{Me}} \mathrm{N} 4\right) \mathrm{NiMe}_{2}$ complex.

\begin{tabular}{|c|c|c|c|c|}
\hline Time, $\mathbf{h}$ & Me-Me, $\%$ & $\mathbf{C D}_{\mathbf{3}}-\mathbf{C H}_{\mathbf{3}}, \boldsymbol{\%}$ & $\mathbf{C D}_{\mathbf{3}}-\mathbf{C D}_{\mathbf{3}} \mathbf{]}, \mathbf{\%}$ & $\mathbf{M e - H / D , ~} \%$ \\
\hline 1 & $14 \pm 1$ & $5 \pm 1$ & 16 & $2 \pm 1$ \\
\hline 2 & $15 \pm 1$ & $6 \pm 1$ & 16 & $2 \pm 1$ \\
\hline 4 & $17 \pm 1$ & $8 \pm 2$ & 18 & $6 \pm 2$ \\
\hline 8 & $21 \pm 2$ & $20 \pm 2$ & 21 & $12 \pm 2$ \\
\hline
\end{tabular}

\section{Reactivity of ( $\left.{ }^{\mathrm{Me}} \mathrm{N} 4\right) \mathrm{Ni}(\mathrm{II}) \mathrm{Me} 2$ with 2 equivalents ${ }^{\mathrm{Ac}_{\mathrm{F}} \mathrm{PF}_{6} .}$}

Table S6. Yields of the products from the reaction of ( $\left.{ }^{\mathrm{Me}} \mathrm{N} 4\right) \mathrm{NiMe}_{2}$ with 2 equivalents of ${ }^{\mathrm{Ac}} \mathrm{FcPF}_{6}$ in $\mathrm{d}_{3}-\mathrm{MeCN}$ at RT.

\begin{tabular}{|c|c|c|}
\hline Time, $\mathbf{h}$ & Me-Me, $\%$ & Me-H/D, $\%$ \\
\hline 0.5 & $88 \pm 1$ & $1 \pm 1$ \\
\hline 8 & $89 \pm 1$ & $1 \pm 1$ \\
\hline
\end{tabular}

Table S7. Yields of the products from the reaction of $\left({ }^{\mathrm{Me}} \mathrm{N} 4\right) \mathrm{NiMe}_{2}$ with 2 equivalents of ${ }^{\mathrm{Ac}} \mathrm{FcPF}_{6}$ and 2 equivalents of TEMPO in $\mathrm{d}_{3}-\mathrm{MeCN}$ at RT.

\begin{tabular}{|c|c|c|}
\hline Time, $\mathbf{h}$ & Me-Me, $\%$ & Me-H/D, $\%$ \\
\hline 0.5 & $86 \pm 2$ & $1 \pm 1$ \\
\hline 8 & $87 \pm 2$ & $1 \pm 1$ \\
\hline
\end{tabular}

Table S8. Yields of the products from the crossover of $\left({ }^{\mathrm{Me}} \mathrm{N} 4\right) \mathrm{NiMe}_{2}$ and $\left({ }^{\mathrm{Me}} \mathrm{N} 4\right) \mathrm{Ni}\left(\mathrm{CD}_{3}\right)_{2}$ with 2 equivalents of ${ }^{\mathrm{Ac}} \mathrm{FcPF}_{6}$ in $\mathrm{d}_{3}-\mathrm{MeCN}$ at $\mathrm{RT}$. The amount of $\mathrm{CD}_{3}-\mathrm{CD}_{3}$ was obtained by subtraction from the overall ethane yield for the non-deuteraed ( $\left.{ }^{\mathrm{Me}} \mathrm{N} 4\right) \mathrm{NiMe}_{2}$ complex

\begin{tabular}{|c|c|c|c|c|}
\hline Time, $\mathbf{h}$ & Me-Me, $\%$ & $\mathbf{C D}_{\mathbf{3}}-\mathbf{C H}_{\mathbf{3}}, \mathbf{\%}$ & {$\left[\mathrm{CD}_{\mathbf{3}}-\mathbf{C D}_{\mathbf{3}}\right], \mathbf{\%}$} & $\mathbf{M e}-\mathbf{H} / \mathbf{D}, \mathbf{\%}$ \\
\hline 0.5 & $41 \pm 2$ & $5 \pm 2$ & 42 & 0 \\
\hline 8 & $41 \pm 3$ & $6 \pm 2$ & 42 & 0 \\
\hline
\end{tabular}




\section{E. Reactivity of [( $\left.\left.{ }^{\mathrm{Me}} \mathrm{N} 4\right) \mathrm{Ni}^{\mathrm{III}} \mathrm{Me}_{2}\right] \mathrm{PF}_{6}$}

Table S9. Yields of the products from the reaction of $\left[\left({ }^{\mathrm{Me}} \mathrm{N} 4\right) \mathrm{Ni}^{\mathrm{III}} \mathrm{Me}_{2}\right] \mathrm{PF}_{6}$ in $\mathrm{d}_{3}-\mathrm{MeCN}$ at $\mathrm{RT}$.

\begin{tabular}{|c|c|c|}
\hline Time, $\mathbf{h}$ & Me-Me, $\%$ & Me-H/D, $\%$ \\
\hline 1 & $14 \pm 2$ & $9 \pm 2$ \\
\hline 2 & $19 \pm 3$ & $10 \pm 3$ \\
\hline 4 & $31 \pm 3$ & $16 \pm 3$ \\
\hline 8 & $43 \pm 3$ & $21 \pm 3$ \\
\hline 24 & $54 \pm 2$ & $30 \pm 1$ \\
\hline
\end{tabular}

Table S10. Yields of the products from the reaction of $\left[\left({ }^{\mathrm{Me}} \mathrm{N} 4\right) \mathrm{Ni}^{\mathrm{III}} \mathrm{Me}_{2}\right] \mathrm{PF}_{6}$ with 1 equivalent of ${ }^{\mathrm{Ac}} \mathrm{FcPF}_{6}$ in $\mathrm{d}_{3}-\mathrm{MeCN}$ at $\mathrm{RT}$.

\begin{tabular}{|c|c|c|}
\hline Time, $\mathbf{h}$ & Me-Me, $\%$ & Me-H/D, $\%$ \\
\hline 1 & $25 \pm 2$ & $1 \pm 1$ \\
\hline 2 & $30 \pm 3$ & $1 \pm 1$ \\
\hline 4 & $38 \pm 3$ & $1 \pm 1$ \\
\hline 8 & $59 \pm 2$ & $1 \pm 1$ \\
\hline 24 & $84 \pm 2$ & $1 \pm 1$ \\
\hline
\end{tabular}

\section{F. Reactivity of ( $\left.{ }^{\mathrm{Me}} \mathrm{N} 4\right) \mathrm{Ni}^{\mathrm{II}} \mathrm{Me}_{2}$ with $\mathrm{CD}_{3} \mathrm{I}$.}

Table S11. Yields of the products from the reaction of $\left({ }^{\mathrm{Me}} \mathrm{N} 4\right) \mathrm{NiMe}_{2}$ with 1 equivalent of $\mathrm{CD}_{3} \mathrm{I}$ in $\mathrm{d}_{3}-\mathrm{MeCN}$ at $\mathrm{RT}$.

\begin{tabular}{|c|c|c|c|}
\hline Time, $\mathbf{h}$ & Me-Me, $\%$ & $\mathbf{C D}_{\mathbf{3}}-\mathbf{C H}_{\mathbf{3}}, \mathbf{\%}$ & $\mathbf{C H}_{\mathbf{3}} \mathbf{- H} \mathbf{D}, \mathbf{\%}$ \\
\hline 1 & 10 & 8 & 2 \\
\hline 2 & 17 & 10 & 2 \\
\hline 4 & 32 & 12 & 2 \\
\hline 8 & 48 & 12 & 2 \\
\hline 24 & 67 & 20 & 2 \\
\hline
\end{tabular}

Table S12. Yields of the products from the reaction of $\left({ }^{\mathrm{Me}} \mathrm{N} 4\right) \mathrm{NiMe}_{2}$ with 1 equivalent of $\mathrm{CD}_{3} \mathrm{I}$ and 2 equivalents of TEMPO in $\mathrm{d}_{3}-\mathrm{MeCN}$ at RT.

\begin{tabular}{|c|c|c|c|c|c|}
\hline $\begin{array}{c}\text { Time, } \\
\mathbf{h}\end{array}$ & $\mathbf{M e - M e , ~ \%}$ & $\mathbf{C D}_{\mathbf{3}}-\mathbf{C H}_{\mathbf{3}}, \mathbf{\%}$ & $\mathbf{C H}_{3}-\mathbf{H} / \mathbf{D}, \mathbf{\%}$ & TEMPO-CD $_{\mathbf{3}}, \mathbf{\%}$ & TEMPO-H, \% \\
\hline 1 & 15 & 8 & 2 & 32 & 0 \\
\hline 4 & 37 & 10 & 3 & 54 & 2 \\
\hline 8 & 54 & 11 & 2 & 74 & 3 \\
\hline 24 & 70 & 8 & 3 & 84 & 7 \\
\hline
\end{tabular}




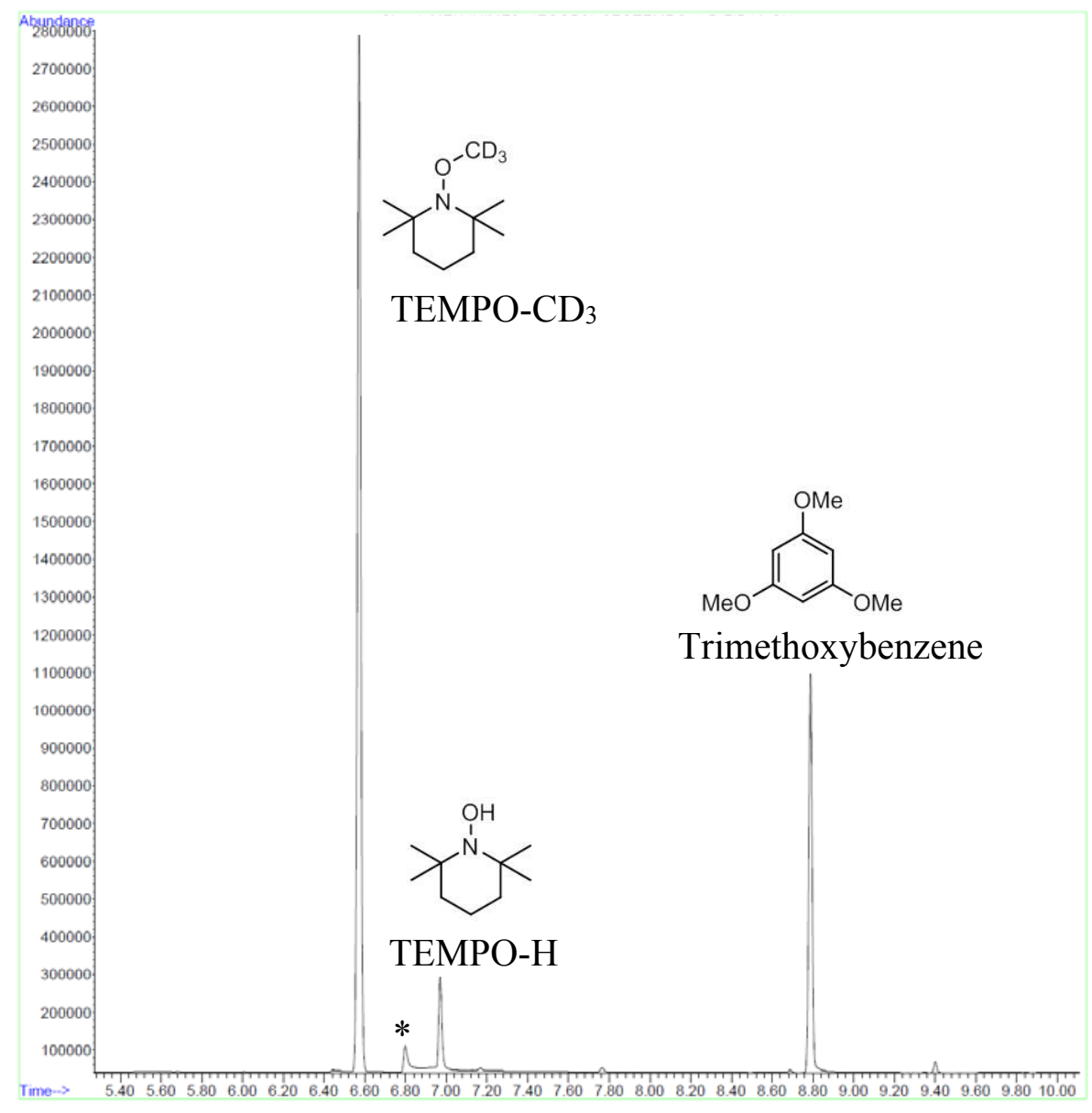

Figure S20. Representative GC chromatogram from the reaction of ( $\left.{ }^{\mathrm{Me}} \mathrm{N} 4\right) \mathrm{NiMe}_{2}$ with 1 equivalents of $\mathrm{CD}_{3} \mathrm{I}$ and 2 equivalents of TEMPO in $\mathrm{d}_{3}-\mathrm{MeCN}$ at RT. The peak marked with an asterisk corresponds to an unidentified TEMPO adduct. The identity of TEMPO-CD $3(\mathrm{~m} / \mathrm{z}=$ 174.18) was confirmed by GC-MS: 


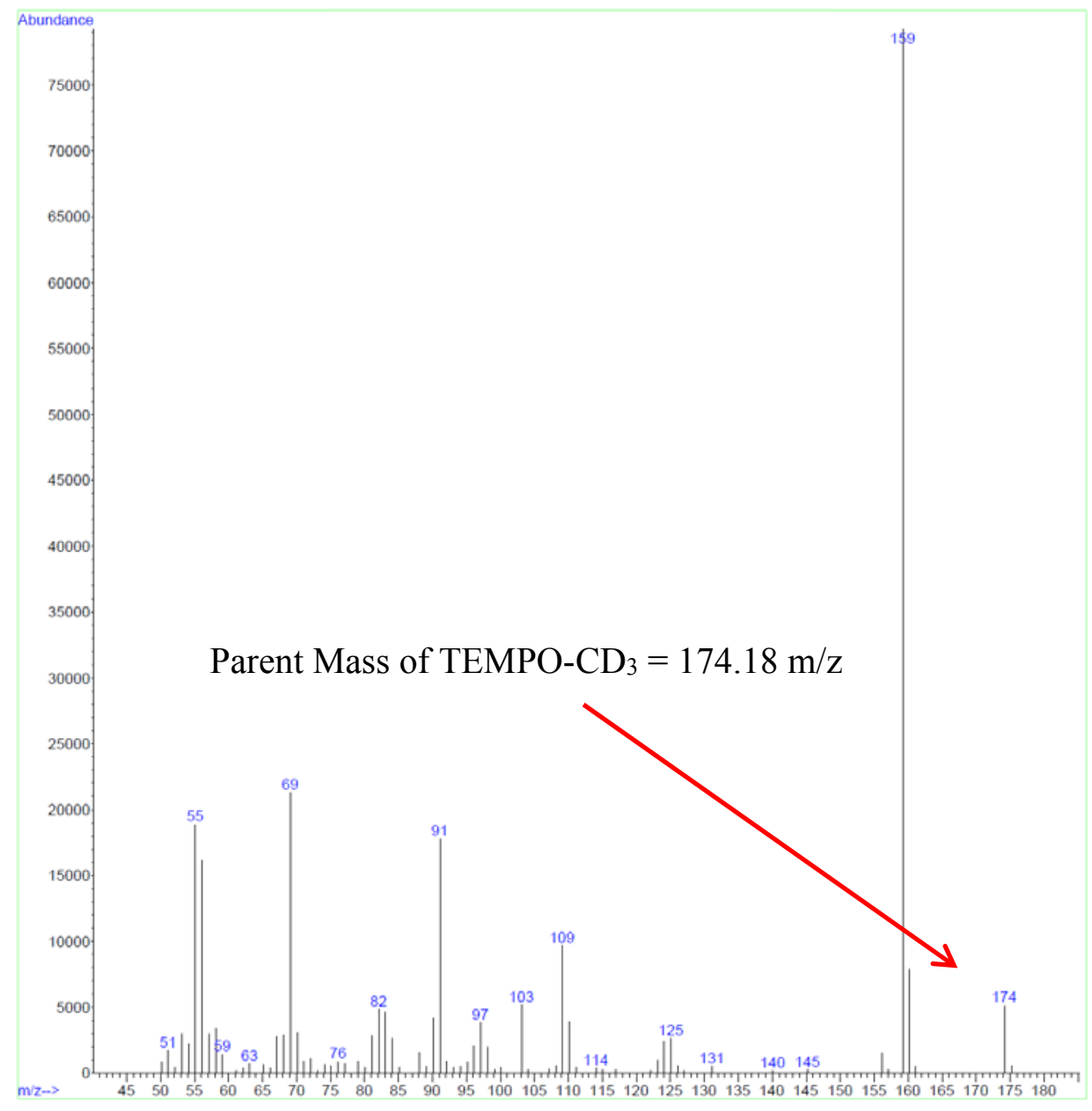

Figure S21. Representative MSD spectrum for the TEMPO-CD 3 peak from the reaction of ( $\left.{ }^{\mathrm{Me}} \mathrm{N} 4\right) \mathrm{NiMe}_{2}$ with 1 equiv $\mathrm{CD}_{3} \mathrm{I}$ and 2 equiv TEMPO in $\mathrm{d}_{3}-\mathrm{MeCN}$ at $\mathrm{RT}$. 

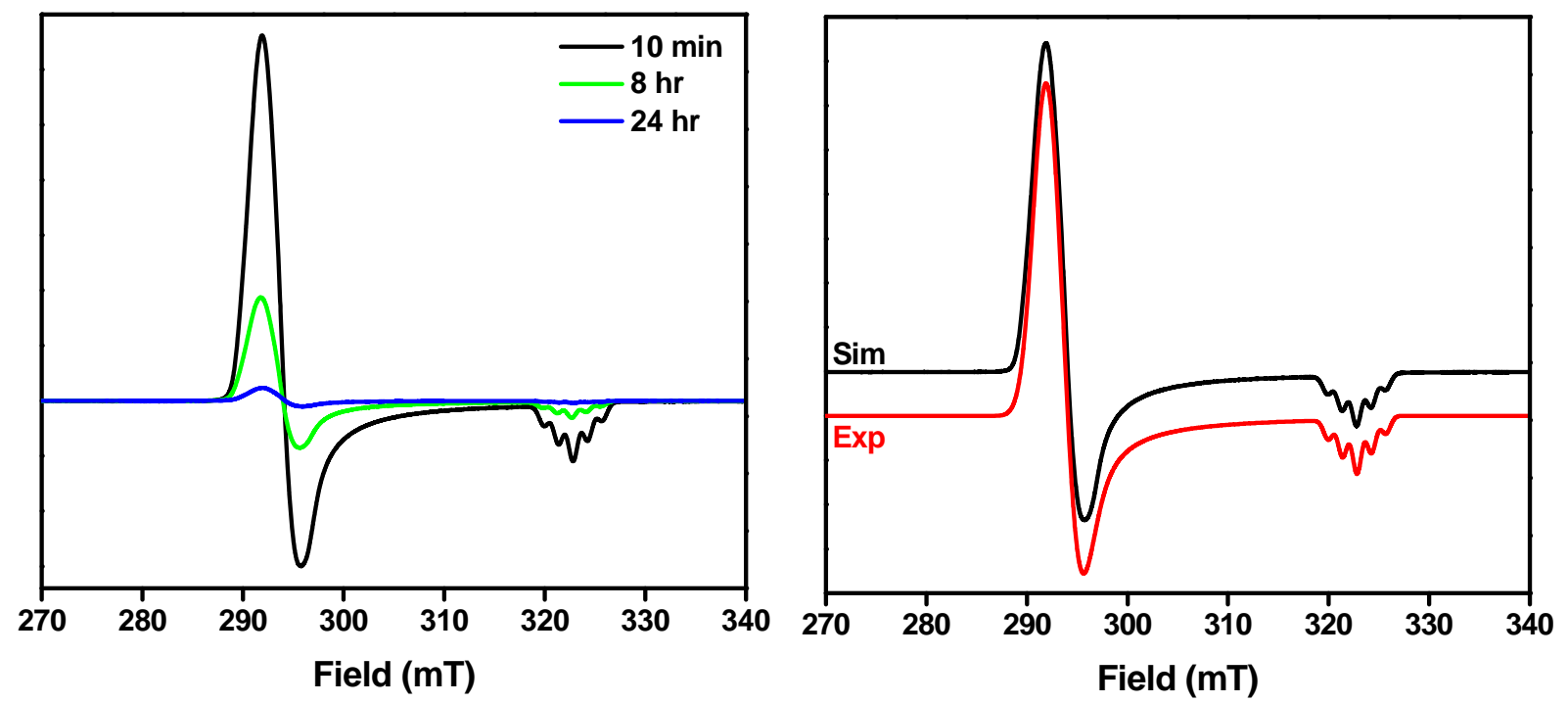

Figure S22. Left: EPR monitoring of the reaction of $\left({ }^{\mathrm{Me}} \mathrm{N} 4\right) \mathrm{NiMe}_{2}$ reacting with 1 equiv of $\mathrm{CD}_{3} \mathrm{I}$ at RT over 24 hours. Right: EPR spectrum and simulation of reaction mixture after 10 min. Simulation used the following parameters: $g_{x}=2.2283, g_{y}=2.2100, g_{z}=2.0137\left(A_{N}=14.30 G\right)$.

\section{G. Reactivity of [( $\left.{ }^{\mathrm{Me}} \mathrm{N} 4\right) \mathrm{Ni}^{\mathrm{III}}$ (cycloneophyl)]PF 6}

Table S13. Yields of the products from the reaction of $\left[\left({ }^{\mathrm{Me}} \mathrm{N} 4\right) \mathrm{Ni}^{\mathrm{III}}\right.$ (cycloneophyl) $] \mathrm{PF} 6$ in $\mathrm{MeCN}$ at RT, as determined by GC-MS.

\begin{tabular}{|c|c|c|}
\hline Time, $\mathbf{h}$ & $\begin{array}{c}\text { 1,1-dimethyl- } \\
\text { benzocyclobutane, } \\
\text { \% }\end{array}$ & tBu-Ph, \% \\
\hline 3 & 9 & 4 \\
\hline 48 & 10 & 8 \\
\hline
\end{tabular}

Table S14. Yields of the products from the reaction of $\left[\left({ }^{\mathrm{Me}} \mathrm{N} 4\right) \mathrm{Ni}^{\mathrm{III}}(\right.$ cycloneophyl) $] \mathrm{PF}_{6}$ with 1 equiv of $\mathrm{NOPF}_{6}$ in $\mathrm{MeCN}$ at RT, as determined by GC-MS.

\begin{tabular}{|c|c|c|}
\hline Time, $\mathbf{h}$ & $\begin{array}{c}\text { 1,1-dimethyl- } \\
\text { benzocyclobutane, } \\
\text { \% }\end{array}$ & tBu-Ph, \% \\
\hline 3 & 17 & 0 \\
\hline 48 & 38 & 0 \\
\hline
\end{tabular}




\section{Catalytic Kumada Coupling Reactions}

General procedure for the Kumada coupling. Inside a nitrogen filled glove box, a small vial equipped with a magnetic stir bar was charged with the corresponding alkyl halide or aryl halide substrate $(0.1 \mathrm{mmol})$, decane as internal standard and $\mathbf{1}$ (1.76 mg, 0.05 equiv.) in THF (5.0 mL). To the stirring solution, the Grignard reagent (1.2 equiv) was added slowly over 1 hour via syringe and the resulting solution was allowed to stir at room temperature for 24 hours. A 1 hour, 2 hour, and 24 hour aliquot $(500 \mu \mathrm{L})$ were taken and the reaction mixture was worked up by quenching with $5 \mathrm{~mL}$ of saturated $\mathrm{NH}_{4} \mathrm{Cl}$ solution, and extracting the mixture with diethyl ether $(3 \times 5 \mathrm{~mL})$. The organic layer was separated and dried over $\mathrm{MgSO}_{4}$. The yield of product(s) was obtained by GC/FID using the internal standard for calibration and reported as an average of at least 3 independent runs. The identity of the products was confirmed by GC/MSD. The investigated cross-coupling reactions shown almost complete consumption of the iodide reagent after 1 hour.

Note: No cross coupled products were observed in the absence of $\mathbf{1}$. 
Kumada Coupling of Iodotoluene and PhMgBr:

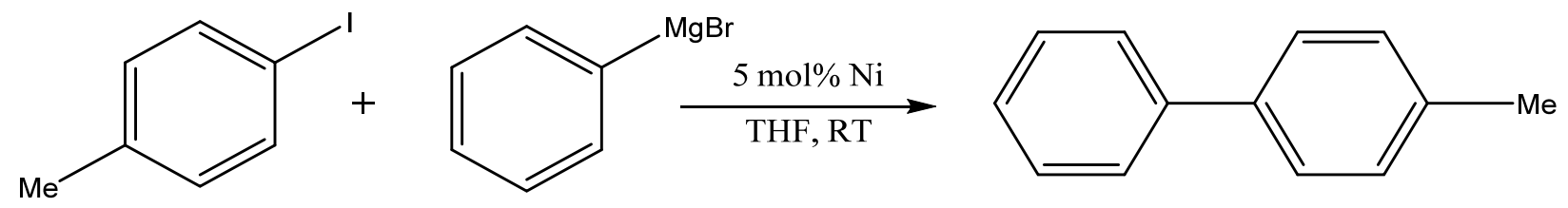

Table S15. Products and yields for the Kumada cross-coupling reaction of iodotoluene and $\mathrm{PhMgBr}$ catalyzed by $5 \mathrm{~mol} \%\left({ }^{\mathrm{Me}} \mathrm{N} 4\right) \mathrm{Ni}^{\mathrm{II}} \mathrm{Me}_{2}$ (reaction time: 1 hour).

\begin{tabular}{|c|c|c|}
\hline Product & Yield \\
\hline Biphenyl & \\
\hline 4-methyl-1,1'-biphenyl & \\
\hline 4,4'-dimethylbiphenyl & $\mathrm{Me}$ & \\
\hline
\end{tabular}




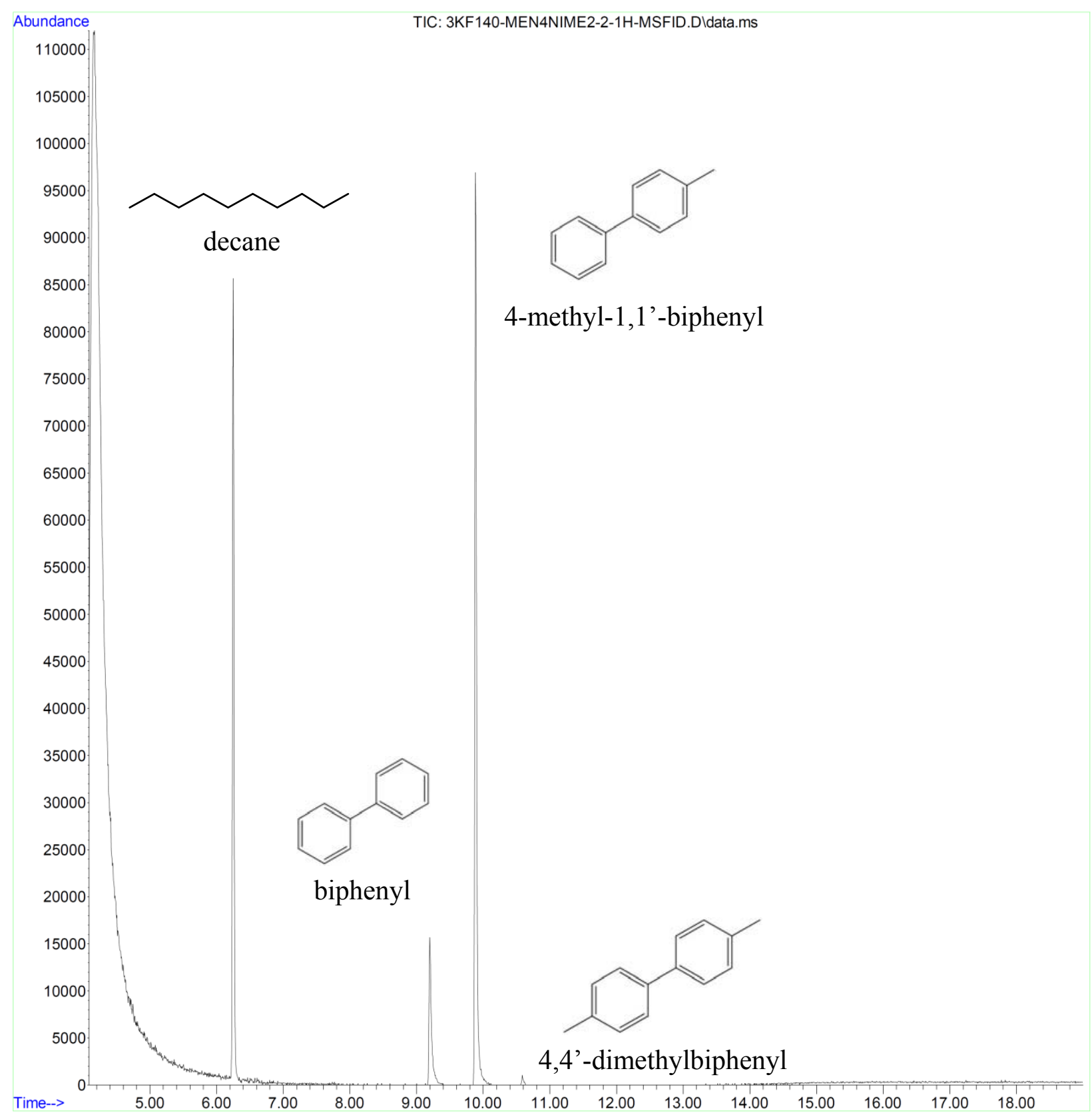

Figure S23. Representative GC chromatogram for the Kumada cross-coupling of Iodotoluene with $\mathrm{PhMgBr}$ catalyzed by $5 \mathrm{~mol} \%\left({ }^{\mathrm{Me}} \mathrm{N} 4\right) \mathrm{Ni}^{\mathrm{II}} \mathrm{Me}_{2}$. 
Kumada Cross-Coupling of Iodotoluene and hexylMgBr:

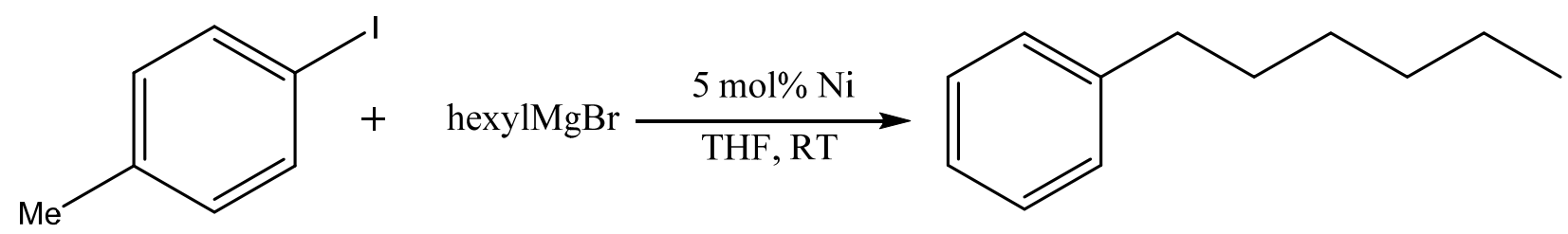

Table S16. Products and yields from the Kumada cross-coupling reactions of Iodotoluene and hexylMgBr catalyzed by $5 \mathrm{~mol} \%\left({ }^{\mathrm{Me}} \mathrm{N} 4\right) \mathrm{Ni}^{\mathrm{II}} \mathrm{Me}_{2}$ (reaction time: 1 hour).

\begin{tabular}{|c|c|c|}
\hline Product & Structure & Yield \\
\hline Toluene & \\
\hline dodecane & \\
\hline 4,4'-Dimethylbiphenyl & Pene & 3 \\
\hline hexylbenzene &
\end{tabular}




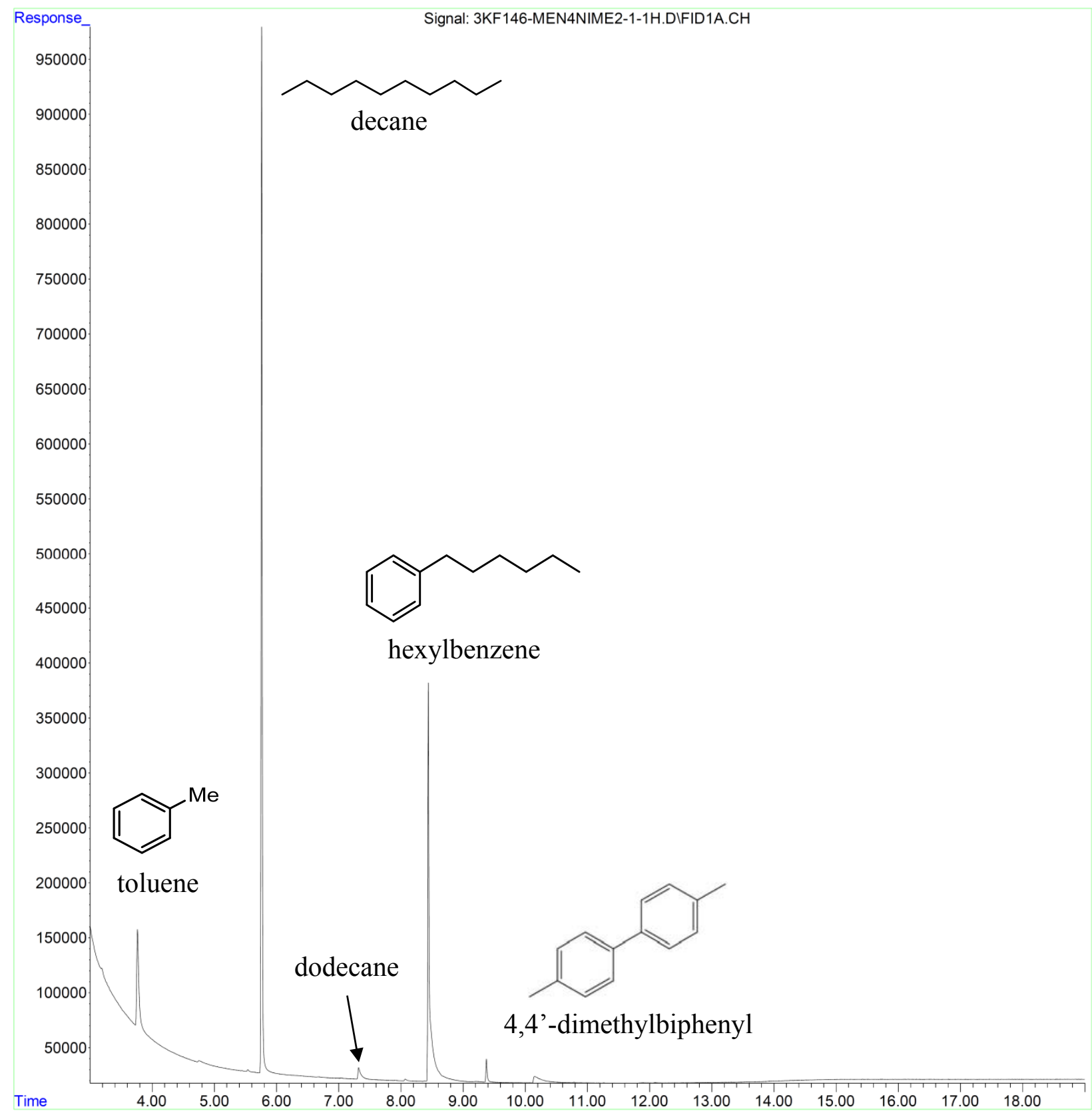

Figure S24. Representative GC chromatogram for the Kumada cross-coupling of Iodotoluene with hexylMgBr catalyzed by $5 \mathrm{~mol} \%$ ( $\left.{ }^{\mathrm{Me}} \mathrm{N} 4\right) \mathrm{Ni}^{\mathrm{II}} \mathrm{Me}_{2}$. 
Kumada Cross-Coupling of Chlorotoluene and PhMgBr:<smiles>Cc1ccc(Cl)cc1</smiles>

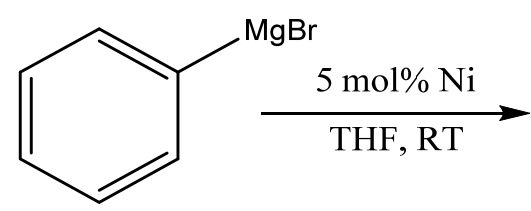

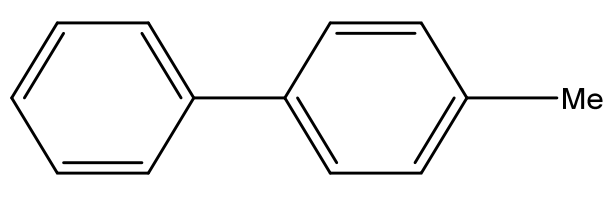

Table S17. Products and yields from the Kumada cross-coupling reactions of Chlorotoluene and $\mathrm{PhMgBr}$ catalyzed by $5 \mathrm{~mol} \%\left({ }^{\mathrm{Me}} \mathrm{N} 4\right) \mathrm{Ni}^{\mathrm{II}} \mathrm{Me}_{2}$ (reaction time: 1 hour).

\begin{tabular}{|c|c|c|}
\hline Product & Yield \\
\hline Toluene & Structure & \\
\hline Chlorotoluene & \\
\hline 4-methyl-1,1'-Biphenyl & \\
\hline 4,4'-Dimethylbiphenyl &
\end{tabular}




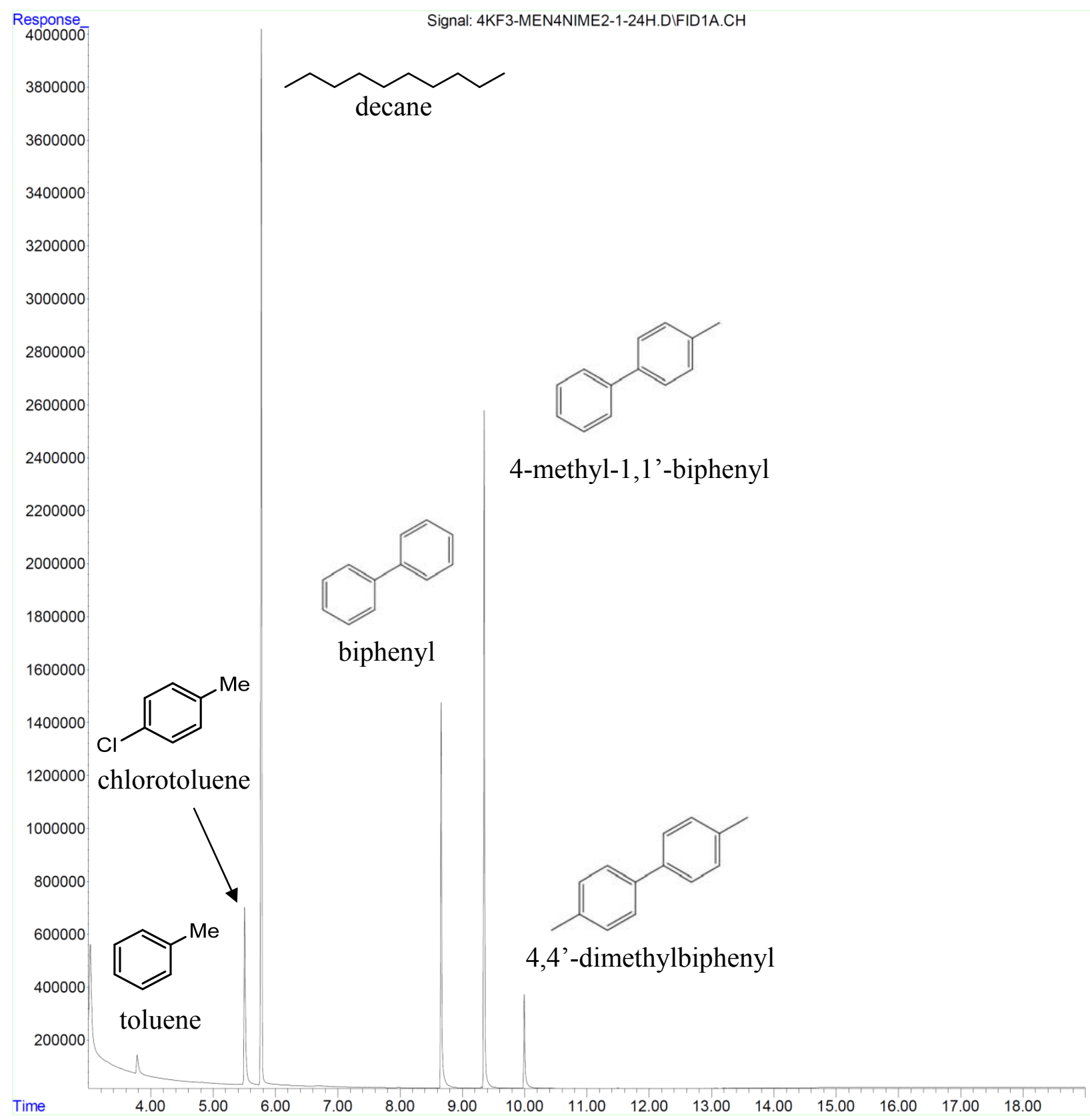

Figure S25. Representative GC chromatogram for the Kumada cross-coupling of chlorotoluene with $\mathrm{PhMgBr}$ catalyzed by $5 \mathrm{~mol} \%\left({ }^{\mathrm{Me}} \mathrm{N} 4\right) \mathrm{Ni}^{\mathrm{II}} \mathrm{Me} 2$. 
Kumada Cross-Coupling of Iodooctane and hexylMgBr:

$$
\text { octyl-I }+ \text { hexylMgBr } \underset{\mathrm{THF}, \mathrm{RT}}{\stackrel{5 \mathrm{~mol} \% \mathrm{Ni}}{\longrightarrow}} \text { tetradecane }
$$

Table S18. Products and yields from the Kumada cross-coupling reactions of iodooctane and hexylMgBr catalyzed by $5 \mathrm{~mol} \%\left({ }^{\mathrm{Me}} \mathrm{N} 4\right) \mathrm{Ni}^{\mathrm{II}} \mathrm{Me}_{2}$ (reaction time: 1 hour).

\begin{tabular}{|c|c|c|}
\hline Product & Structure & Yield \\
\hline Octane & $\mathrm{C}_{12} \mathrm{H}_{26}$ & 47 \\
\hline hexyl-THF & $\mathrm{C}_{14} \mathrm{H}_{30}$ & 4 \\
\hline dodecane & & 20 \\
\hline tetradecane & & \\
\hline
\end{tabular}




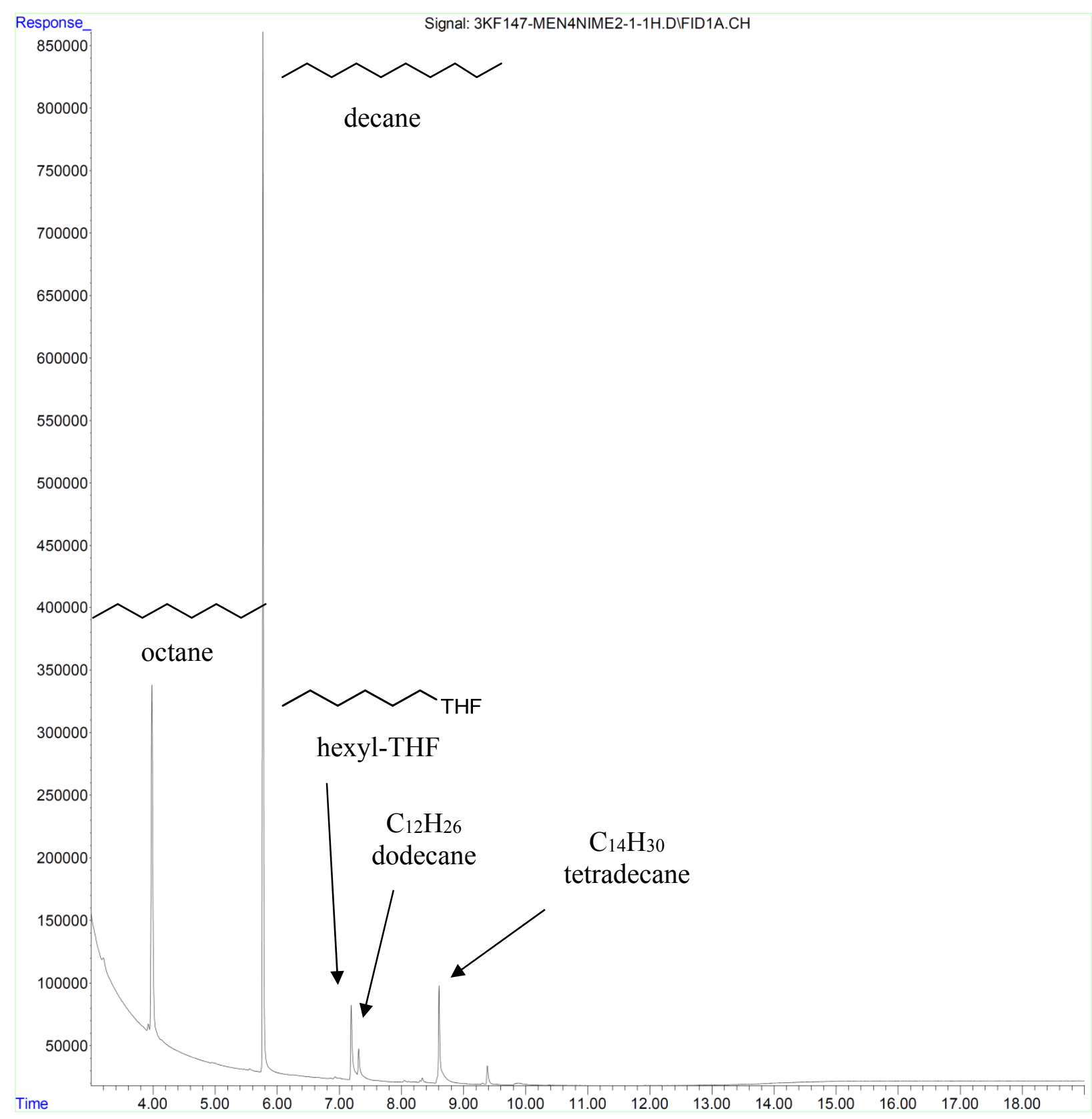

Figure S26. Representative GC chromatogram for the Kumada cross-coupling of iodooctane with hexylMgBr catalyzed by $5 \mathrm{~mol} \%\left({ }^{\mathrm{Me}} \mathrm{N} 4\right) \mathrm{Ni}^{\mathrm{II}} \mathrm{Me}_{2}$. 


\section{X-ray structure determinations of Ni Complexes}

X-ray quality crystals of ( $\left.{ }^{\mathrm{Me}} \mathrm{N} 4\right) \mathrm{NiMe}_{2}$ were obtained by slow pentane diffusion into an ether solution at $-35^{\circ} \mathrm{C}$. X-ray quality crystals of $\left[\left({ }^{\mathrm{Me}} \mathrm{N} 4\right) \mathrm{NiMe}_{2}\right] \mathrm{BPh} 4,\left({ }^{\mathrm{Me}} \mathrm{N} 4\right) \mathrm{Ni}^{\mathrm{II}}$ (cycloneophyl), and $\left[\left({ }^{\mathrm{Me}} \mathrm{N} 4\right) \mathrm{Ni}^{\mathrm{III}}\right.$ (cycloneophyl)]PF6 were obtained by slow pentane vapor diffusion into a tetrahydrofuran solution at $-35^{\circ} \mathrm{C}$. The $\left[\left({ }^{\mathrm{Me}} \mathrm{N} 4\right) \mathrm{Ni}^{\mathrm{II}}(\mathrm{MeCN})_{2}\right]\left(\mathrm{PF}_{6}\right)_{2}$ complex was obtained from the reaction mixture of a reductive elimination reactivity of 3 in $\mathrm{MeCN}$, upon slow diethyl ether vapor diffusion at $-35^{\circ} \mathrm{C}$. Suitable crystals of appropriate dimensions were mounted on Mitgen loops in random orientations. Preliminary examination and data collection were performed using a Bruker Kappa Apex-II Charge Coupled Device (CCD) Detector system single crystal X-Ray diffractometer equipped with an Oxford Cryostream LT device. Data were collected using graphite monochromated Mo K $\alpha$ radiation $(\lambda=0.71073 \AA)$ from a fine focus sealed tube X-Ray source. Preliminary unit cell constants were determined with a set of 36 narrow frame scans. Typical data sets consist of a combination of $\omega$ and $\phi$ scan frames with typical scan width of $0.5^{\circ}$ and counting time of 15-30 seconds/frame at a crystal to detector distance of $\sim 4.0 \mathrm{~cm}$. The collected frames were integrated using an orientation matrix determined from the narrow frame scans. Apex II and SAINT software packages (Bruker Analytical X-Ray, Madison, WI, 2008) were used for data collection and data integration. Analysis of the integrated data did not show any decay. Final cell constants were determined by global refinement of reflections from the complete data set. Data were corrected for systematic errors using SADABS (Bruker Analytical $X$-Ray, Madison, WI, 2008) based on the Laue symmetry using equivalent reflections.

Structure solutions and refinement were carried out using the SHELXTL- PLUS software package (Sheldrick, G. M. (2008), Bruker-SHELXTL, Acta Cryst. A64,112-122). The structures were refined with full matrix least-squares refinement by minimizing $\Sigma \mathrm{w}\left(\mathrm{F}_{\mathrm{o}}^{2}-\mathrm{F}_{\mathrm{c}}^{2}\right)^{2}$. All nonhydrogen atoms were refined anisotropically to convergence. Typically, $\mathrm{H}$ atoms are added at the calculated positions in the final refinement cycles.

Acknowledgement: Funding from the National Science Foundation (MRI, CHE-0420497) for the purchase of the ApexII diffractometer is acknowledged. 


\section{$\mathrm{X}$-ray structure determination of ( $\left.{ }^{\mathrm{Me}} \mathrm{N} 4\right) \mathrm{NiMe}_{2}$}

Table S19. Crystal data and structure refinement for $\operatorname{lm} 13514$.

Identification code

Empirical formula

Formula weight

Temperature

Wavelength

Crystal syst

em

Space group

Unit cell dimensions

Volume

Z

Density (calculated)

Absorption coefficient

$\mathrm{F}(000)$

Crystal size

Theta range for data collection

Index ranges

Reflections collected

Independent reflections

Completeness to theta $=25.242^{\circ}$

Absorption correction

Max. and min. transmission

Refinement method

Data / restraints / parameters

Goodness-of-fit on $\mathrm{F}^{2}$

Final $R$ indices [I $>2 \operatorname{sigma}(\mathrm{I})]$

$\mathrm{R}$ indices (all data)

Extinction coefficient

Largest diff. peak and hole
113514/1t/x8/JS-091514-01

$\mathrm{C} 18 \mathrm{H} 26 \mathrm{~N} 4 \mathrm{Ni}$

357.14

100(2) K

$0.71073 \AA$

Monoclinic

$\mathrm{C} 2 / \mathrm{c}$

$\mathrm{a}=14.2510(7) \AA$ $\alpha=90^{\circ}$.

$\mathrm{b}=13.1814(5) \AA$

$\beta=98.280(3)^{\circ}$.

$\mathrm{c}=9.2333(4) \AA$

$\gamma=90^{\circ}$.

1716.38(13) $\AA^{3}$

4

$1.382 \mathrm{Mg} / \mathrm{m}^{3}$

$1.135 \mathrm{~mm}^{-1}$

760

$0.289 \times 0.204 \times 0.078 \mathrm{~mm}^{3}$

2.115 to $28.316^{\circ}$.

$-18 \leq \mathrm{h} \leq 18,-17 \leq \mathrm{k} \leq 17,-12 \leq \mathrm{l} \leq 11$

10169

$2135[\mathrm{R}(\mathrm{int})=0.0360]$

$100.0 \%$

Semi-empirical from equivalents

0.8621 and 0.7705

Full-matrix least-squares on $\mathrm{F}^{2}$

$2135 / 0 / 215$

1.016

$\mathrm{R} 1=0.0263, \mathrm{wR} 2=0.0544$

$\mathrm{R} 1=0.0358, \mathrm{wR}_{2}=0.0591$

$\mathrm{n} / \mathrm{a}$

0.241 and -0.162 e. $\AA^{-3}$ 
Table S20. Bond lengths $[\AA]$ and angles $\left[{ }^{\circ}\right]$ for $\operatorname{lm} 13514$.

\begin{tabular}{|c|c|c|c|}
\hline $\mathrm{Ni}(1)-\mathrm{C}(1)$ & $1.913(7)$ & $\mathrm{C}\left(1^{\prime}\right)-\mathrm{H}\left(1^{\prime} 1\right)$ & 0.9800 \\
\hline $\mathrm{Ni}(1)-\mathrm{C}(1) \# 1$ & $1.913(7)$ & $\mathrm{C}\left(1^{\prime}\right)-\mathrm{H}\left(1^{\prime} 2\right)$ & 0.9800 \\
\hline $\mathrm{Ni}(1)-\mathrm{N}(1)$ & $1.973(6)$ & $\mathrm{C}\left(1^{\prime}\right)-\mathrm{H}\left(1^{\prime} 3\right)$ & 0.9800 \\
\hline $\mathrm{Ni}(1)-\mathrm{N}(1) \# 1$ & $1.973(6)$ & $C\left(2^{\prime}\right)-C\left(3^{\prime}\right)$ & $1.371(5)$ \\
\hline $\mathrm{N}(1)-\mathrm{C}(6)$ & $1.342(6)$ & $C\left(2^{\prime}\right)-C\left(8^{\prime}\right)$ & $1.519(5)$ \\
\hline $\mathrm{N}(1)-\mathrm{C}(2)$ & $1.351(5)$ & $C\left(3^{\prime}\right)-C\left(4^{\prime}\right)$ & $1.389(6$ \\
\hline $\mathrm{N}(2)-\mathrm{C}(8) \# 1$ & $1.447(4)$ & $\mathrm{C}\left(3^{\prime}\right)-\mathrm{H}\left(3^{\prime}\right)$ & 0.9500 \\
\hline $\mathrm{N}(2)-\mathrm{C}(9)$ & $1.457(4)$ & $C\left(4^{\prime}\right)-C\left(5^{\prime}\right)$ & $1.348(9$ \\
\hline $\mathrm{N}(2)-\mathrm{C}(7)$ & $1.461(4)$ & $\mathrm{C}\left(4^{\prime}\right)-\mathrm{H}\left(4^{\prime}\right)$ & 0.9500 \\
\hline $\mathrm{C}(1)-\mathrm{H}(1 \mathrm{~A})$ & 0.9800 & $\mathrm{C}\left(5^{\prime}\right)-\mathrm{C}\left(6^{\prime}\right)$ & $1.414(5$ \\
\hline $\mathrm{C}(1)-\mathrm{H}(1 \mathrm{~B})$ & 0.9800 & $\mathrm{C}\left(5^{\prime}\right)-\mathrm{H}\left(5^{\prime}\right)$ & 0.9500 \\
\hline $\mathrm{C}(1)-\mathrm{H}(1 \mathrm{C})$ & 0.9800 & $C\left(6^{\prime}\right)-C\left(7^{\prime}\right)$ & $1.509(4$ \\
\hline $\mathrm{C}(2)-\mathrm{C}(3)$ & $1.381(4)$ & $\mathrm{C}\left(7^{\prime}\right)-\mathrm{H}\left(7^{\prime} \mathrm{A}\right)$ & 0.9900 \\
\hline$C(2)-C(8)$ & $1.520(5)$ & $\mathrm{C}\left(7^{\prime}\right)-\mathrm{H}\left(7^{\prime} \mathrm{B}\right)$ & 0.9900 \\
\hline$C(3)-C(4)$ & $1.395(4)$ & $\mathrm{C}\left(8^{\prime}\right)-\mathrm{N}\left(2^{\prime}\right) \# 1$ & $1.441(6$ \\
\hline $\mathrm{C}(3)-\mathrm{H}(3)$ & 0.9500 & $\mathrm{C}\left(8^{\prime}\right)-\mathrm{H}\left(8^{\prime} \mathrm{A}\right)$ & 0.9900 \\
\hline$C(4)-C(5)$ & $1.402(5)$ & $\mathrm{C}\left(8^{\prime}\right)-\mathrm{H}\left(8^{\prime} \mathrm{B}\right)$ & 0.9900 \\
\hline $\mathrm{C}(4)-\mathrm{H}(4)$ & 0.9500 & $\mathrm{C}\left(9^{\prime}\right)-\mathrm{H}\left(9^{\prime} \mathrm{A}\right)$ & 0.9800 \\
\hline$C(5)-C(6)$ & $1.389(6)$ & $\mathrm{C}\left(9^{\prime}\right)-\mathrm{H}\left(9^{\prime} \mathrm{B}\right)$ & 0.9800 \\
\hline $\mathrm{C}(5)-\mathrm{H}(5)$ & 0.9500 & $\mathrm{C}\left(9^{\prime}\right)-\mathrm{H}\left(9^{\prime} \mathrm{C}\right)$ & 0.9800 \\
\hline$C(6)-C(7)$ & $1.493(4)$ & $C(1)-N i(1)-C(1) \# 1$ & $91.1(7)$ \\
\hline $\mathrm{C}(7)-\mathrm{H}(7 \mathrm{~A})$ & 0.9900 & $\mathrm{C}(1)-\mathrm{Ni}(1)-\mathrm{N}(1)$ & $91.6(3)$ \\
\hline $\mathrm{C}(7)-\mathrm{H}(7 \mathrm{~B})$ & 0.9900 & $\mathrm{C}(1) \# 1-\mathrm{Ni}(1)-\mathrm{N}(1)$ & $173.5(7)$ \\
\hline $\mathrm{C}(8)-\mathrm{N}(2) \# 1$ & $1.447(4)$ & $\mathrm{C}(1)-\mathrm{Ni}(1)-\mathrm{N}(1) \# 1$ & $173.5(7)$ \\
\hline $\mathrm{C}(8)-\mathrm{H}(8 \mathrm{~A})$ & 0.9900 & $\mathrm{C}(1) \# 1-\mathrm{Ni}(1)-\mathrm{N}(1) \# 1$ & $91.6(3)$ \\
\hline $\mathrm{C}(8)-\mathrm{H}(8 \mathrm{~B})$ & 0.9900 & $\mathrm{~N}(1)-\mathrm{Ni}(1)-\mathrm{N}(1) \# 1$ & $86.5(3)$ \\
\hline $\mathrm{C}(9)-\mathrm{H}(9 \mathrm{~A})$ & 0.9800 & $\mathrm{C}(6)-\mathrm{N}(1)-\mathrm{C}(2)$ & $119.4(4)$ \\
\hline C(9)-H(9B) & 0.9800 & $\mathrm{C}(6)-\mathrm{N}(1)-\mathrm{Ni}(1)$ & $120.2(3)$ \\
\hline $\mathrm{C}(9)-\mathrm{H}(9 \mathrm{C})$ & 0.9800 & $\mathrm{C}(2)-\mathrm{N}(1)-\mathrm{Ni}(1)$ & $120.5(4)$ \\
\hline $\mathrm{Ni}\left(1^{\prime}\right)-\mathrm{C}\left(1^{\prime}\right) \# 1$ & $1.915(7)$ & $\mathrm{C}(8) \# 1-\mathrm{N}(2)-\mathrm{C}(9)$ & $112.8(3)$ \\
\hline $\mathrm{Ni}\left(1^{\prime}\right)-\mathrm{C}\left(1^{\prime}\right)$ & $1.915(7)$ & $\mathrm{C}(8) \# 1-\mathrm{N}(2)-\mathrm{C}(7)$ & 119.1(3) \\
\hline $\mathrm{Ni}\left(1^{\prime}\right)-\mathrm{N}\left(1^{\prime}\right)$ & $1.950(7)$ & $\mathrm{C}(9)-\mathrm{N}(2)-\mathrm{C}(7)$ & $114.4(3)$ \\
\hline $\mathrm{Ni}\left(1^{\prime}\right)-\mathrm{N}\left(1^{\prime}\right) \# 1$ & $1.950(7)$ & $\mathrm{Ni}(1)-\mathrm{C}(1)-\mathrm{H}(1 \mathrm{~A})$ & 109.5 \\
\hline $\mathrm{N}\left(1^{\prime}\right)-\mathrm{C}\left(6^{\prime}\right)$ & $1.341(4)$ & $\mathrm{Ni}(1)-\mathrm{C}(1)-\mathrm{H}(1 \mathrm{~B})$ & 109.5 \\
\hline $\mathrm{N}\left(1^{\prime}\right)-\mathrm{C}\left(2^{\prime}\right)$ & $1.353(6)$ & $\mathrm{H}(1 \mathrm{~A})-\mathrm{C}(1)-\mathrm{H}(1 \mathrm{~B})$ & 109.5 \\
\hline $\mathrm{N}\left(2^{\prime}\right)-\mathrm{C}\left(8^{\prime}\right) \# 1$ & $1.441(6)$ & $\mathrm{Ni}(1)-\mathrm{C}(1)-\mathrm{H}(1 \mathrm{C})$ & 109.5 \\
\hline $\mathrm{N}\left(2^{\prime}\right)-\mathrm{C}\left(9^{\prime}\right)$ & $1.460(5)$ & $\mathrm{H}(1 \mathrm{~A})-\mathrm{C}(1)-\mathrm{H}(1 \mathrm{C})$ & 109.5 \\
\hline $\mathrm{N}\left(2^{\prime}\right)-\mathrm{C}\left(7^{\prime}\right)$ & $1.470(4)$ & $\mathrm{H}(1 \mathrm{~B})-\mathrm{C}(1)-\mathrm{H}(1 \mathrm{C})$ & 109.5 \\
\hline
\end{tabular}




\begin{tabular}{|c|c|c|c|}
\hline $\mathrm{N}(1)-\mathrm{C}(2)-\mathrm{C}(3)$ & $121.4(4)$ & $\mathrm{C}\left(6^{\prime}\right)-\mathrm{N}\left(1^{\prime}\right)-\mathrm{Ni}\left(1^{\prime}\right)$ & $119.7(4)$ \\
\hline $\mathrm{N}(1)-\mathrm{C}(2)-\mathrm{C}(8)$ & $118.6(4)$ & $\mathrm{C}\left(2^{\prime}\right)-\mathrm{N}\left(1^{\prime}\right)-\mathrm{Ni}\left(1^{\prime}\right)$ & $121.9(4)$ \\
\hline$C(3)-C(2)-C(8)$ & $120.0(4)$ & $\mathrm{C}\left(8^{\prime}\right) \# 1-\mathrm{N}\left(2^{\prime}\right)-\mathrm{C}\left(9^{\prime}\right)$ & $115.1(3)$ \\
\hline$C(2)-C(3)-C(4)$ & $119.6(3)$ & $\mathrm{C}\left(8^{\prime}\right) \# 1-\mathrm{N}\left(2^{\prime}\right)-\mathrm{C}\left(7^{\prime}\right)$ & $118.5(3)$ \\
\hline $\mathrm{C}(2)-\mathrm{C}(3)-\mathrm{H}(3)$ & 120.2 & $\mathrm{C}\left(9^{\prime}\right)-\mathrm{N}\left(2^{\prime}\right)-\mathrm{C}\left(7^{\prime}\right)$ & $112.1(3)$ \\
\hline $\mathrm{C}(4)-\mathrm{C}(3)-\mathrm{H}(3)$ & 120.2 & $\mathrm{Ni}\left(1^{\prime}\right)-\mathrm{C}\left(1^{\prime}\right)-\mathrm{H}\left(1^{\prime} 1\right)$ & 109.5 \\
\hline$C(3)-C(4)-C(5)$ & $118.7(4)$ & $\mathrm{Ni}\left(1^{\prime}\right)-\mathrm{C}\left(1^{\prime}\right)-\mathrm{H}\left(1^{\prime} 2\right)$ & 109.5 \\
\hline $\mathrm{C}(3)-\mathrm{C}(4)-\mathrm{H}(4)$ & 120.6 & $\mathrm{H}\left(1^{\prime} 1\right)-\mathrm{C}\left(1^{\prime}\right)-\mathrm{H}\left(1^{\prime} 2\right)$ & 109.5 \\
\hline $\mathrm{C}(5)-\mathrm{C}(4)-\mathrm{H}(4)$ & 120.6 & Ni(1')-C(1')-H(1'3) & 109.5 \\
\hline$C(6)-C(5)-C(4)$ & $118.2(5)$ & $\mathrm{H}\left(1^{\prime} 1\right)-\mathrm{C}\left(1^{\prime}\right)-\mathrm{H}\left(1^{\prime} 3\right)$ & 109.5 \\
\hline $\mathrm{C}(6)-\mathrm{C}(5)-\mathrm{H}(5)$ & 120.9 & $\mathrm{H}\left(1^{\prime} 2\right)-\mathrm{C}\left(1^{\prime}\right)-\mathrm{H}\left(1^{\prime} 3\right)$ & 109.5 \\
\hline $\mathrm{C}(4)-\mathrm{C}(5)-\mathrm{H}(5)$ & 120.9 & $\mathrm{~N}\left(1^{\prime}\right)-\mathrm{C}\left(2^{\prime}\right)-\mathrm{C}\left(3^{\prime}\right)$ & $122.3(5)$ \\
\hline $\mathrm{N}(1)-\mathrm{C}(6)-\mathrm{C}(5)$ & $122.6(4)$ & $\mathrm{N}\left(1^{\prime}\right)-\mathrm{C}\left(2^{\prime}\right)-\mathrm{C}\left(8^{\prime}\right)$ & $117.2(4)$ \\
\hline $\mathrm{N}(1)-\mathrm{C}(6)-\mathrm{C}(7)$ & $118.5(3)$ & $\mathrm{C}\left(3^{\prime}\right)-\mathrm{C}\left(2^{\prime}\right)-\mathrm{C}\left(8^{\prime}\right)$ & $120.5(3)$ \\
\hline$C(5)-C(6)-C(7)$ & $118.9(4)$ & $\mathrm{C}\left(2^{\prime}\right)-\mathrm{C}\left(3^{\prime}\right)-\mathrm{C}\left(4^{\prime}\right)$ & $118.7(4)$ \\
\hline $\mathrm{N}(2)-\mathrm{C}(7)-\mathrm{C}(6)$ & $117.7(2)$ & $\mathrm{C}\left(2^{\prime}\right)-\mathrm{C}\left(3^{\prime}\right)-\mathrm{H}\left(3^{\prime}\right)$ & 120.7 \\
\hline N(2)-C(7)-H(7A) & 107.9 & $\mathrm{C}\left(4^{\prime}\right)-\mathrm{C}\left(3^{\prime}\right)-\mathrm{H}\left(3^{\prime}\right)$ & 120.7 \\
\hline $\mathrm{C}(6)-\mathrm{C}(7)-\mathrm{H}(7 \mathrm{~A})$ & 107.9 & $\mathrm{C}\left(5^{\prime}\right)-\mathrm{C}\left(4^{\prime}\right)-\mathrm{C}\left(3^{\prime}\right)$ & $120.5(5)$ \\
\hline N(2)-C(7)-H(7B) & 107.9 & $\mathrm{C}\left(5^{\prime}\right)-\mathrm{C}\left(4^{\prime}\right)-\mathrm{H}\left(4^{\prime}\right)$ & 119.8 \\
\hline $\mathrm{C}(6)-\mathrm{C}(7)-\mathrm{H}(7 \mathrm{~B})$ & 107.9 & $\mathrm{C}\left(3^{\prime}\right)-\mathrm{C}\left(4^{\prime}\right)-\mathrm{H}\left(4^{\prime}\right)$ & 119.8 \\
\hline H(7A)-C(7)-H(7B) & 107.2 & $C\left(4^{\prime}\right)-C\left(5^{\prime}\right)-C\left(6^{\prime}\right)$ & $118.3(4)$ \\
\hline N(2)\#1-C(8)-C(2) & $118.0(4)$ & $\mathrm{C}\left(4^{\prime}\right)-\mathrm{C}\left(5^{\prime}\right)-\mathrm{H}\left(5^{\prime}\right)$ & 120.9 \\
\hline $\mathrm{N}(2) \# 1-\mathrm{C}(8)-\mathrm{H}(8 \mathrm{~A})$ & 107.8 & $\mathrm{C}\left(6^{\prime}\right)-\mathrm{C}\left(5^{\prime}\right)-\mathrm{H}\left(5^{\prime}\right)$ & 120.9 \\
\hline $\mathrm{C}(2)-\mathrm{C}(8)-\mathrm{H}(8 \mathrm{~A})$ & 107.8 & $\mathrm{~N}\left(1^{\prime}\right)-\mathrm{C}\left(6^{\prime}\right)-\mathrm{C}\left(5^{\prime}\right)$ & $121.9(4)$ \\
\hline $\mathrm{N}(2) \# 1-\mathrm{C}(8)-\mathrm{H}(8 \mathrm{~B})$ & 107.8 & $\mathrm{~N}\left(1^{\prime}\right)-\mathrm{C}\left(6^{\prime}\right)-\mathrm{C}\left(7^{\prime}\right)$ & $118.5(4)$ \\
\hline $\mathrm{C}(2)-\mathrm{C}(8)-\mathrm{H}(8 \mathrm{~B})$ & 107.8 & $C\left(5^{\prime}\right)-C\left(6^{\prime}\right)-C\left(7^{\prime}\right)$ & $119.6(4)$ \\
\hline $\mathrm{H}(8 \mathrm{~A})-\mathrm{C}(8)-\mathrm{H}(8 \mathrm{~B})$ & 107.1 & $\mathrm{~N}\left(2^{\prime}\right)-\mathrm{C}\left(7^{\prime}\right)-\mathrm{C}\left(6^{\prime}\right)$ & $117.6(3)$ \\
\hline N(2)-C(9)-H(9A) & 109.5 & $\mathrm{~N}\left(2^{\prime}\right)-\mathrm{C}\left(7^{\prime}\right)-\mathrm{H}\left(7^{\prime} \mathrm{A}\right)$ & 107.9 \\
\hline N(2)-C(9)-H(9B) & 109.5 & $\mathrm{C}\left(6^{\prime}\right)-\mathrm{C}\left(7^{\prime}\right)-\mathrm{H}\left(7^{\prime} \mathrm{A}\right)$ & 107.9 \\
\hline $\mathrm{H}(9 \mathrm{~A})-\mathrm{C}(9)-\mathrm{H}(9 \mathrm{~B})$ & 109.5 & $\mathrm{~N}\left(2^{\prime}\right)-\mathrm{C}\left(7^{\prime}\right)-\mathrm{H}\left(7^{\prime} \mathrm{B}\right)$ & 107.9 \\
\hline N(2)-C(9)-H(9C) & 109.5 & $\mathrm{C}\left(6^{\prime}\right)-\mathrm{C}\left(7^{\prime}\right)-\mathrm{H}\left(7^{\prime} \mathrm{B}\right)$ & 107.9 \\
\hline $\mathrm{H}(9 \mathrm{~A})-\mathrm{C}(9)-\mathrm{H}(9 \mathrm{C})$ & 109.5 & $\mathrm{H}\left(7^{\prime} \mathrm{A}\right)-\mathrm{C}\left(7^{\prime}\right)-\mathrm{H}\left(7^{\prime} \mathrm{B}\right)$ & 107.2 \\
\hline H(9B)-C(9)-H(9C) & 109.5 & $\mathrm{~N}\left(2^{\prime}\right) \# 1-\mathrm{C}\left(8^{\prime}\right)-\mathrm{C}\left(2^{\prime}\right)$ & $117.8(3)$ \\
\hline C(1')\#1-Ni(1')-C(1') & $89.2(3)$ & $\mathrm{N}\left(2^{\prime}\right) \# 1-\mathrm{C}\left(8^{\prime}\right)-\mathrm{H}\left(8^{\prime} \mathrm{A}\right)$ & 107.9 \\
\hline C(1')\#1-Ni(1')-N(1') & 177.1(3) & $\mathrm{C}\left(2^{\prime}\right)-\mathrm{C}\left(8^{\prime}\right)-\mathrm{H}\left(8^{\prime} \mathrm{A}\right)$ & 107.9 \\
\hline C(1')-Ni(1')-N(1') & $92.1(2)$ & $\mathrm{N}\left(2^{\prime}\right) \# 1-\mathrm{C}\left(8^{\prime}\right)-\mathrm{H}\left(8^{\prime} \mathrm{B}\right)$ & 107.9 \\
\hline $\mathrm{C}\left(1^{\prime}\right) \# 1-\mathrm{Ni}\left(1^{\prime}\right)-\mathrm{N}\left(1^{\prime}\right) \# 1$ & $92.1(2)$ & $\mathrm{C}\left(2^{\prime}\right)-\mathrm{C}\left(8^{\prime}\right)-\mathrm{H}\left(8^{\prime} \mathrm{B}\right)$ & 107.9 \\
\hline C(1')-Ni(1')-N(1')\#1 & 177.1(3) & $\mathrm{H}\left(8^{\prime} \mathrm{A}\right)-\mathrm{C}\left(8^{\prime}\right)-\mathrm{H}\left(8^{\prime} \mathrm{B}\right)$ & 107.2 \\
\hline $\mathrm{N}\left(1^{\prime}\right)-\mathrm{Ni}\left(1^{\prime}\right)-\mathrm{N}\left(1^{\prime}\right) \# 1$ & $86.8(3)$ & $\mathrm{N}\left(2^{\prime}\right)-\mathrm{C}\left(9^{\prime}\right)-\mathrm{H}\left(9^{\prime} \mathrm{A}\right)$ & 109.5 \\
\hline $\mathrm{C}\left(6^{\prime}\right)-\mathrm{N}\left(1^{\prime}\right)-\mathrm{C}\left(2^{\prime}\right)$ & $118.3(5)$ & $\mathrm{N}\left(2^{\prime}\right)-\mathrm{C}\left(9^{\prime}\right)-\mathrm{H}\left(9^{\prime} \mathrm{B}\right)$ & 109.5 \\
\hline
\end{tabular}



$\mathrm{H}\left(9^{\prime} \mathrm{A}\right)-\mathrm{C}\left(9^{\prime}\right)-\mathrm{H}\left(9^{\prime} \mathrm{B}\right) \quad 109.5$
$\mathrm{H}\left(9^{\prime} \mathrm{A}\right)-\mathrm{C}\left(9^{\prime}\right)-\mathrm{H}\left(9^{\prime} \mathrm{C}\right) \quad 109.5$
$\mathrm{N}\left(2^{\prime}\right)-\mathrm{C}\left(9^{\prime}\right)-\mathrm{H}\left(9^{\prime} \mathrm{C}\right) \quad 109.5$
$\mathrm{H}\left(9^{\prime} \mathrm{B}\right)-\mathrm{C}\left(9^{\prime}\right)-\mathrm{H}\left(9^{\prime} \mathrm{C}\right) \quad 109.5$

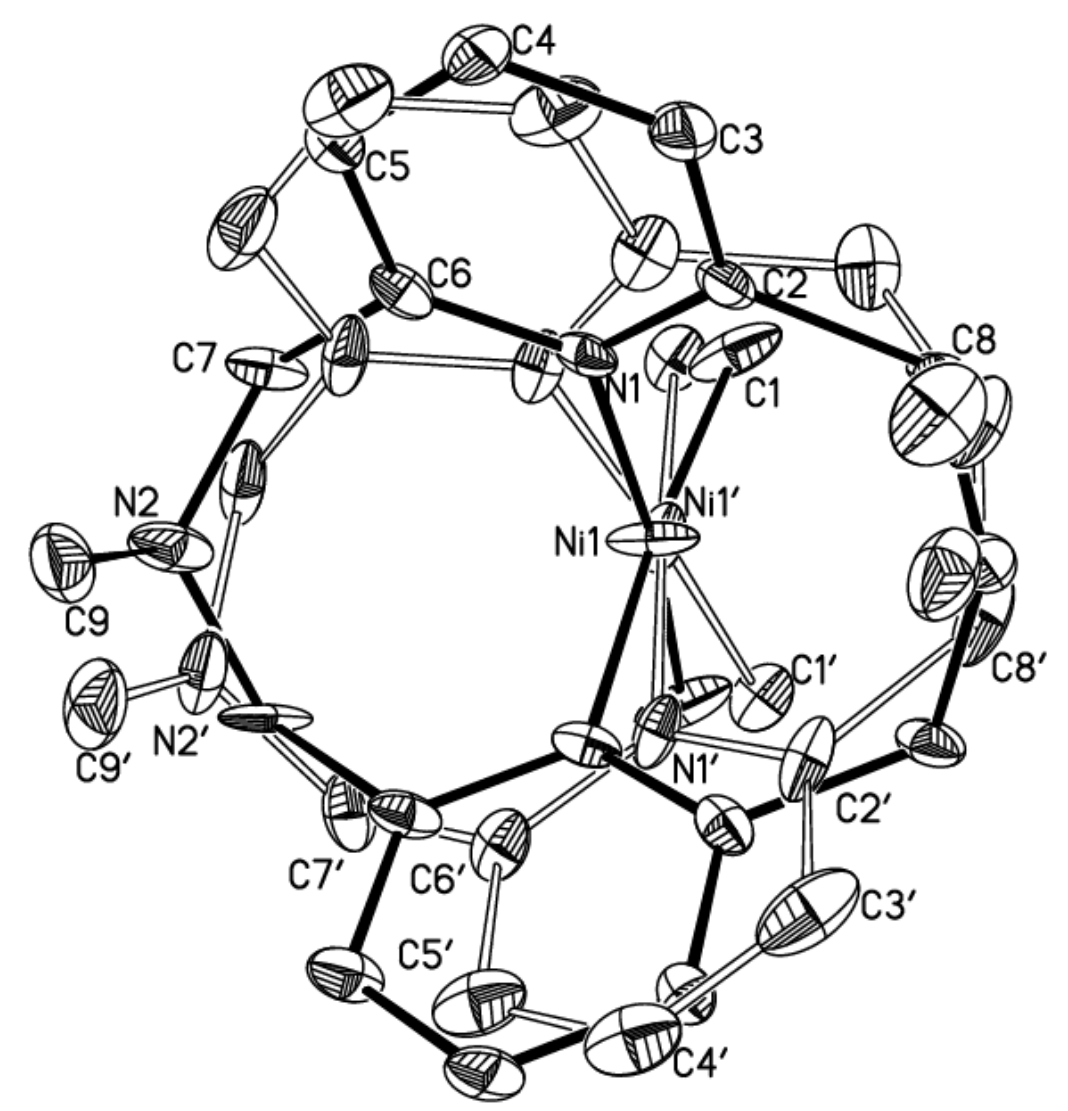

Figure S27. Projection view of $\operatorname{lm} 13514$ with 50\% thermal ellipsoids. 


\section{$\mathrm{X}$-ray structure determination of [( $\left.\left.{ }^{\mathrm{Me}} \mathrm{N} 4\right) \mathrm{NiMe}_{2}\right] \mathrm{BPh}_{4}$}

Table S21. Crystal data and structure refinement for $1 \mathrm{~m} 8714$.

Identification code

Empirical formula

Formula weight

Temperature

Wavelength

Crystal system

Space group

Unit cell dimensions

Volume

Z

Density (calculated)

Absorption coefficient

$\mathrm{F}(000)$

Crystal size

Theta range for data collection

Index ranges

Reflections collected

Independent reflections

Completeness to theta $=25.242^{\circ}$

Absorption correction

Max. and min. transmission

Refinement method

Data / restraints / parameters

Goodness-of-fit on $\mathrm{F}^{2}$

Final $\mathrm{R}$ indices [I $>2 \operatorname{sigma}(\mathrm{I})]$

$\mathrm{R}$ indices (all data)

Extinction coefficient

Largest diff. peak and hole
18714_sq/lt/x8/JS-061614-01

$\mathrm{C}_{46} \mathrm{H}_{54} \mathrm{~B} \mathrm{~N} 4 \mathrm{Ni} \mathrm{O}$

748.45

100(2) K

$0.71073 \AA$

Triclinic

$\mathrm{P} \overline{1}$

$\mathrm{a}=8.9600(7) \AA \quad \alpha=88.779(4)^{\circ}$.

$\mathrm{b}=14.2611(10) \AA \quad \beta=75.985(4)^{\circ}$.

$\mathrm{c}=16.7826(12) \AA \quad \gamma=73.867(4)^{\circ}$.

1996.3(3) $\AA^{3}$

2

$1.245 \mathrm{Mg} / \mathrm{m}^{3}$

$0.526 \mathrm{~mm}^{-1}$

798

$0.323 \times 0.224 \times 0.149 \mathrm{~mm}^{3}$

1.252 to $27.608^{\circ}$.

$-11 \leq \mathrm{h} \leq 11,-18 \leq \mathrm{k} \leq 18,-21 \leq \mathrm{l} \leq 21$

40240

$9164[\mathrm{R}(\mathrm{int})=0.0406]$

$99.7 \%$

Semi-empirical from equivalents

0.8621 and 0.7941

Full-matrix least-squares on $\mathrm{F}^{2}$

9164 / 1 / 487

1.031

$\mathrm{R} 1=0.0389, \mathrm{wR} 2=0.0904$

$\mathrm{R} 1=0.0518, \mathrm{wR} 2=0.0966$

$\mathrm{n} / \mathrm{a}$

0.532 and -0.437 e. $\AA^{-3}$ 
Table S22. Bond lengths $[\AA]$ and angles $\left[{ }^{\circ}\right]$ for $\operatorname{lm} 8714$.

$\begin{array}{llll}\mathrm{Ni}(1)-\mathrm{C}(2) & 1.9782(19) & \mathrm{C}(9)-\mathrm{H}(9 \mathrm{~A}) & 0.9900 \\ \mathrm{Ni}(1)-\mathrm{N}(1) & 1.9792(14) & \mathrm{C}(9)-\mathrm{H}(9 \mathrm{~B}) & 0.9900 \\ \mathrm{Ni}(1)-\mathrm{C}(1) & 1.987(2) & \mathrm{C}(10)-\mathrm{C}(11) & 1.381(3) \\ \mathrm{Ni}(1)-\mathrm{C}\left(1^{\prime}\right) & 1.994(9) & \mathrm{C}(11)-\mathrm{C}(12) & 1.384(3) \\ \mathrm{Ni}(1)-\mathrm{N}(2) & 1.9958(15) & \mathrm{C}(11)-\mathrm{H}(11) & 0.9500 \\ \mathrm{Ni}(1)-\mathrm{N}(4) & 2.2399(14) & \mathrm{C}(12)-\mathrm{C}(13) & 1.388(3) \\ \mathrm{Ni}(1)-\mathrm{N}(3) & 2.2519(14) & \mathrm{C}(12)-\mathrm{H}(12) & 0.9500 \\ \mathrm{~N}(1)-\mathrm{C}(7) & 1.340(2) & \mathrm{C}(13)-\mathrm{C}(14) & 1.378(3) \\ \mathrm{N}(1)-\mathrm{C}(3) & 1.342(2) & \mathrm{C}(13)-\mathrm{H}(13) & 0.9500 \\ \mathrm{~N}(2)-\mathrm{C}(10) & 1.343(2) & \mathrm{C}(14)-\mathrm{C}(15) & 1.506(3) \\ \mathrm{N}(2)-\mathrm{C}(14) & 1.343(2) & \mathrm{C}(15)-\mathrm{H}(15 \mathrm{~A}) & 0.9900 \\ \mathrm{~N}(3)-\mathrm{C}(17) & 1.471(2) & \mathrm{C}(15)-\mathrm{H}(15 \mathrm{~B}) & 0.9900 \\ \mathrm{~N}(3)-\mathrm{C}(8) & 1.478(2) & \mathrm{C}(16)-\mathrm{H}(16 \mathrm{~A}) & 0.9900 \\ \mathrm{~N}(3)-\mathrm{C}(9) & 1.485(2) & \mathrm{C}(16)-\mathrm{H}(16 \mathrm{~B}) & 0.9900 \\ \mathrm{~N}(4)-\mathrm{C}(18) & 1.479(2) & \mathrm{C}(17)-\mathrm{H}(17 \mathrm{~A}) & 0.9800 \\ \mathrm{~N}(4)-\mathrm{C}(15) & 1.480(2) & \mathrm{C}(17)-\mathrm{H}(17 \mathrm{~B}) & 0.9800 \\ \mathrm{~N}(4)-\mathrm{C}(16) & 1.484(2) & \mathrm{C}(17)-\mathrm{H}(17 \mathrm{C}) & 0.9800 \\ \mathrm{C}(1)-\mathrm{H}(1 \mathrm{~A}) & 0.9800 & \mathrm{C}(18)-\mathrm{H}(18 \mathrm{~A}) & 0.9800 \\ \mathrm{C}(1)-\mathrm{H}(1 \mathrm{~B}) & 0.9800 & \mathrm{C}(18)-\mathrm{H}(18 \mathrm{~B}) & 0.9800 \\ \mathrm{C}(1)-\mathrm{H}(1 \mathrm{C}) & 0.9800 & \mathrm{C}(18)-\mathrm{H}(18 \mathrm{C}) & 0.9800 \\ \mathrm{C}\left(1^{\prime}\right)-\mathrm{H}\left(1^{\prime} 1\right) & 0.9800 & \mathrm{O}(1 \mathrm{~S})-\mathrm{C}(1 \mathrm{~S}) & 1.417(3) \\ \mathrm{C}\left(1^{\prime}\right)-\mathrm{H}(1 ' 2) & 0.9800 & \mathrm{O}(1 \mathrm{~S})-\mathrm{C}(4 \mathrm{~S}) & 1.432(3) \\ \mathrm{C}\left(1^{\prime}\right)-\mathrm{H}(1 ' 3) & 0.9800 & \mathrm{C}(1 \mathrm{~S})-\mathrm{C}(2 \mathrm{~S}) & 1.497(3) \\ \mathrm{C}(2)-\mathrm{H}(2 \mathrm{~A}) & 0.9800 & \mathrm{C}(1 \mathrm{~S})-\mathrm{H}(1 \mathrm{SA}) & 0.9900 \\ \mathrm{C}(2)-\mathrm{H}(2 \mathrm{~B}) & 0.9800 & \mathrm{C}(1 \mathrm{~S})-\mathrm{H}(1 \mathrm{SB}) & 0.9900 \\ \mathrm{C}(2)-\mathrm{H}(2 \mathrm{C}) & 0.9800 & \mathrm{C}(2 \mathrm{~S})-\mathrm{C}(3 \mathrm{~S}) & 1.507(3) \\ \mathrm{C}(3)-\mathrm{C}(4) & 1.381(2) & \mathrm{C}(2 \mathrm{~S})-\mathrm{H}(2 \mathrm{SA}) & 0.9900 \\ \mathrm{C}(3)-\mathrm{C}(16) & 1.506(2) & \mathrm{C}(2 \mathrm{~S})-\mathrm{H}(2 \mathrm{SB}) & 0.9900 \\ \mathrm{C}(4)-\mathrm{C}(5) & \mathrm{C}(3 \mathrm{~S})-\mathrm{C}(4 \mathrm{~S}) & 1.506(4) \\ \mathrm{C}(4)-\mathrm{H}(4) & \mathrm{C}(3 \mathrm{~S})-\mathrm{H}(3 \mathrm{SA}) & 0.9900 \\ \mathrm{C}(5)-\mathrm{C}(6) & \mathrm{C}(3 \mathrm{~S})-\mathrm{H}(3 \mathrm{SB}) & 0.9900 \\ \mathrm{C}(5)-\mathrm{H}(5) & \mathrm{C}(4 \mathrm{~S})-\mathrm{H}(4 \mathrm{SA}) & 0.9900 \\ \mathrm{C}(6)-\mathrm{C}(7) & \mathrm{C}(4 \mathrm{~S})-\mathrm{H}(4 \mathrm{SB}) & 0.9900 \\ \mathrm{C}(6)-\mathrm{H}(6) & \mathrm{C}(19)-\mathrm{C}(24) & 1.396(3) \\ \mathrm{C}(7)-\mathrm{C}(8) & \mathrm{C}(19)-\mathrm{C}(20) & 1.402(3) \\ \mathrm{C}(8)-\mathrm{H}(8 \mathrm{~A}) & \mathrm{C}(19)-\mathrm{B}(1) & 1.645(2) \\ \mathrm{C}(8)-\mathrm{H}(8 \mathrm{~B}) & \mathrm{C}(20)-\mathrm{C}(21) & 1.394(3) \\ \mathrm{C}(9)-\mathrm{C}(10) & \mathrm{C}(20)-\mathrm{H}(20) & 0.9500 \\ & 0.9500 & & \end{array}$




\begin{tabular}{|c|c|c|c|}
\hline$C(21)-C(22)$ & $1.380(3)$ & $\mathrm{C}(41)-\mathrm{C}(42)$ & $1.391(3)$ \\
\hline $\mathrm{C}(21)-\mathrm{H}(21)$ & 0.9500 & $\mathrm{C}(41)-\mathrm{H}(41)$ & 0.9500 \\
\hline$C(22)-C(23)$ & $1.380(3)$ & $\mathrm{C}(42)-\mathrm{H}(42)$ & 0.9500 \\
\hline $\mathrm{C}(22)-\mathrm{H}(22)$ & 0.9500 & $\mathrm{C}(2)-\mathrm{Ni}(1)-\mathrm{N}(1)$ & $93.26(7)$ \\
\hline$C(23)-C(24)$ & $1.396(3)$ & $\mathrm{C}(2)-\mathrm{Ni}(1)-\mathrm{C}(1)$ & $84.68(17)$ \\
\hline $\mathrm{C}(23)-\mathrm{H}(23)$ & 0.9500 & $\mathrm{~N}(1)-\mathrm{Ni}(1)-\mathrm{C}(1)$ & $176.34(10)$ \\
\hline $\mathrm{C}(24)-\mathrm{H}(24)$ & 0.9500 & $\mathrm{C}(2)-\mathrm{Ni}(1)-\mathrm{C}\left(1^{\prime}\right)$ & $71.9(8)$ \\
\hline$C(25)-C(30)$ & $1.400(2)$ & $\mathrm{N}(1)-\mathrm{Ni}(1)-\mathrm{C}\left(1^{\prime}\right)$ & $165.1(8)$ \\
\hline$C(25)-C(26)$ & $1.403(2)$ & $\mathrm{C}(2)-\mathrm{Ni}(1)-\mathrm{N}(2)$ & $176.88(7)$ \\
\hline $\mathrm{C}(25)-\mathrm{B}(1)$ & $1.642(2)$ & $\mathrm{N}(1)-\mathrm{Ni}(1)-\mathrm{N}(2)$ & $84.19(6)$ \\
\hline$C(26)-C(27)$ & $1.389(3)$ & $\mathrm{C}(1)-\mathrm{Ni}(1)-\mathrm{N}(2)$ & $97.96(16)$ \\
\hline $\mathrm{C}(26)-\mathrm{H}(26)$ & 0.9500 & $\mathrm{C}\left(1^{\prime}\right)-\mathrm{Ni}(1)-\mathrm{N}(2)$ & $110.7(8)$ \\
\hline $\mathrm{C}(27)-\mathrm{C}(28)$ & $1.385(3)$ & $\mathrm{C}(2)-\mathrm{Ni}(1)-\mathrm{N}(4)$ & $97.74(7)$ \\
\hline $\mathrm{C}(27)-\mathrm{H}(27)$ & 0.9500 & $\mathrm{~N}(1)-\mathrm{Ni}(1)-\mathrm{N}(4)$ & $80.22(6)$ \\
\hline $\mathrm{C}(28)-\mathrm{C}(29)$ & $1.383(3)$ & $\mathrm{C}(1)-\mathrm{Ni}(1)-\mathrm{N}(4)$ & $103.03(9)$ \\
\hline $\mathrm{C}(28)-\mathrm{H}(28)$ & 0.9500 & $\mathrm{C}\left(1^{\prime}\right)-\mathrm{Ni}(1)-\mathrm{N}(4)$ & $101.5(6)$ \\
\hline$C(29)-C(30)$ & $1.387(3)$ & $\mathrm{N}(2)-\mathrm{Ni}(1)-\mathrm{N}(4)$ & $80.07(6)$ \\
\hline C(29)-H(29) & 0.9500 & $\mathrm{C}(2)-\mathrm{Ni}(1)-\mathrm{N}(3)$ & $101.88(7)$ \\
\hline $\mathrm{C}(30)-\mathrm{H}(30)$ & 0.9500 & $\mathrm{~N}(1)-\mathrm{Ni}(1)-\mathrm{N}(3)$ & $80.42(6)$ \\
\hline$C(31)-C(36)$ & $1.400(3)$ & $\mathrm{C}(1)-\mathrm{Ni}(1)-\mathrm{N}(3)$ & $97.02(10)$ \\
\hline$C(31)-C(32)$ & $1.404(3)$ & $\mathrm{C}\left(1^{\prime}\right)-\mathrm{Ni}(1)-\mathrm{N}(3)$ & $102.0(6)$ \\
\hline $\mathrm{C}(31)-\mathrm{B}(1)$ & $1.644(3)$ & $\mathrm{N}(2)-\mathrm{Ni}(1)-\mathrm{N}(3)$ & $79.51(6)$ \\
\hline$C(32)-C(33)$ & $1.396(3)$ & $\mathrm{N}(4)-\mathrm{Ni}(1)-\mathrm{N}(3)$ & $153.07(6)$ \\
\hline $\mathrm{C}(32)-\mathrm{H}(32)$ & 0.9500 & $\mathrm{C}(7)-\mathrm{N}(1)-\mathrm{C}(3)$ & $120.86(15)$ \\
\hline$C(33)-C(34)$ & $1.382(3)$ & $\mathrm{C}(7)-\mathrm{N}(1)-\mathrm{Ni}(1)$ & $119.74(12)$ \\
\hline $\mathrm{C}(33)-\mathrm{H}(33)$ & 0.9500 & $\mathrm{C}(3)-\mathrm{N}(1)-\mathrm{Ni}(1)$ & $118.96(11)$ \\
\hline$C(34)-C(35)$ & $1.382(3)$ & $\mathrm{C}(10)-\mathrm{N}(2)-\mathrm{C}(14)$ & $120.20(16)$ \\
\hline $\mathrm{C}(34)-\mathrm{H}(34)$ & 0.9500 & $\mathrm{C}(10)-\mathrm{N}(2)-\mathrm{Ni}(1)$ & $119.06(12)$ \\
\hline$C(35)-C(36)$ & $1.397(3)$ & $\mathrm{C}(14)-\mathrm{N}(2)-\mathrm{Ni}(1)$ & $119.18(12)$ \\
\hline $\mathrm{C}(35)-\mathrm{H}(35)$ & 0.9500 & $\mathrm{C}(17)-\mathrm{N}(3)-\mathrm{C}(8)$ & $109.27(14)$ \\
\hline $\mathrm{C}(36)-\mathrm{H}(36)$ & 0.9500 & $\mathrm{C}(17)-\mathrm{N}(3)-\mathrm{C}(9)$ & $108.37(13)$ \\
\hline$C(37)-C(42)$ & $1.400(2)$ & $\mathrm{C}(8)-\mathrm{N}(3)-\mathrm{C}(9)$ & $112.00(14)$ \\
\hline$C(37)-C(38)$ & $1.401(2)$ & $\mathrm{C}(17)-\mathrm{N}(3)-\mathrm{Ni}(1)$ & $116.59(11)$ \\
\hline $\mathrm{C}(37)-\mathrm{B}(1)$ & $1.638(2)$ & $\mathrm{C}(8)-\mathrm{N}(3)-\mathrm{Ni}(1)$ & $106.15(10)$ \\
\hline$C(38)-C(39)$ & $1.390(3)$ & $\mathrm{C}(9)-\mathrm{N}(3)-\mathrm{Ni}(1)$ & $104.45(10)$ \\
\hline $\mathrm{C}(38)-\mathrm{H}(38)$ & 0.9500 & $C(18)-N(4)-C(15)$ & $108.70(14)$ \\
\hline$C(39)-C(40)$ & $1.385(3)$ & $\mathrm{C}(18)-\mathrm{N}(4)-\mathrm{C}(16)$ & $108.27(14)$ \\
\hline $\mathrm{C}(39)-\mathrm{H}(39)$ & 0.9500 & $C(15)-N(4)-C(16)$ & $112.40(14)$ \\
\hline$C(40)-C(41)$ & $1.385(3)$ & $\mathrm{C}(18)-\mathrm{N}(4)-\mathrm{Ni}(1)$ & $116.36(11)$ \\
\hline $\mathrm{C}(40)-\mathrm{H}(40)$ & 0.9500 & $\mathrm{C}(15)-\mathrm{N}(4)-\mathrm{Ni}(1)$ & $106.94(10)$ \\
\hline
\end{tabular}




\begin{tabular}{|c|c|c|c|}
\hline$C(16)-N(4)-N i(1)$ & $104.21(10)$ & $\mathrm{N}(3)-\mathrm{C}(9)-\mathrm{C}(10)$ & $111.81(14)$ \\
\hline $\mathrm{Ni}(1)-\mathrm{C}(1)-\mathrm{H}(1 \mathrm{~A})$ & 109.5 & $\mathrm{~N}(3)-\mathrm{C}(9)-\mathrm{H}(9 \mathrm{~A})$ & 109.3 \\
\hline $\mathrm{Ni}(1)-\mathrm{C}(1)-\mathrm{H}(1 \mathrm{~B})$ & 109.5 & $\mathrm{C}(10)-\mathrm{C}(9)-\mathrm{H}(9 \mathrm{~A})$ & 109.3 \\
\hline $\mathrm{H}(1 \mathrm{~A})-\mathrm{C}(1)-\mathrm{H}(1 \mathrm{~B})$ & 109.5 & $\mathrm{~N}(3)-\mathrm{C}(9)-\mathrm{H}(9 \mathrm{~B})$ & 109.3 \\
\hline Ni(1)-C(1)-H(1C) & 109.5 & $\mathrm{C}(10)-\mathrm{C}(9)-\mathrm{H}(9 \mathrm{~B})$ & 109.3 \\
\hline $\mathrm{H}(1 \mathrm{~A})-\mathrm{C}(1)-\mathrm{H}(1 \mathrm{C})$ & 109.5 & $\mathrm{H}(9 \mathrm{~A})-\mathrm{C}(9)-\mathrm{H}(9 \mathrm{~B})$ & 107.9 \\
\hline $\mathrm{H}(1 \mathrm{~B})-\mathrm{C}(1)-\mathrm{H}(1 \mathrm{C})$ & 109.5 & $\mathrm{~N}(2)-\mathrm{C}(10)-\mathrm{C}(11)$ & $121.49(17)$ \\
\hline Ni(1)-C(1')-H(1'1) & 109.5 & $\mathrm{~N}(2)-\mathrm{C}(10)-\mathrm{C}(9)$ & $114.91(15)$ \\
\hline Ni(1)-C(1')-H(1'2) & 109.5 & $C(11)-C(10)-C(9)$ & $123.56(16)$ \\
\hline $\mathrm{H}\left(1^{\prime} 1\right)-\mathrm{C}\left(1^{\prime}\right)-\mathrm{H}\left(1^{\prime} 2\right)$ & 109.5 & $C(10)-C(11)-C(12)$ & $118.48(18)$ \\
\hline Ni(1)-C(1')-H(1'3) & 109.5 & $\mathrm{C}(10)-\mathrm{C}(11)-\mathrm{H}(11)$ & 120.8 \\
\hline $\mathrm{H}\left(1^{\prime} 1\right)-\mathrm{C}\left(1^{\prime}\right)-\mathrm{H}\left(1^{\prime} 3\right)$ & 109.5 & $\mathrm{C}(12)-\mathrm{C}(11)-\mathrm{H}(11)$ & 120.8 \\
\hline $\mathrm{H}\left(1^{\prime} 2\right)-\mathrm{C}\left(1^{\prime}\right)-\mathrm{H}\left(1^{\prime} 3\right)$ & 109.5 & $C(11)-C(12)-C(13)$ & $119.78(19)$ \\
\hline Ni(1)-C(2)-H(2A) & 109.5 & $\mathrm{C}(11)-\mathrm{C}(12)-\mathrm{H}(12)$ & 120.1 \\
\hline Ni(1)-C(2)-H(2B) & 109.5 & $\mathrm{C}(13)-\mathrm{C}(12)-\mathrm{H}(12)$ & 120.1 \\
\hline $\mathrm{H}(2 \mathrm{~A})-\mathrm{C}(2)-\mathrm{H}(2 \mathrm{~B})$ & 109.5 & $C(14)-C(13)-C(12)$ & $118.82(18)$ \\
\hline Ni(1)-C(2)-H(2C) & 109.5 & $\mathrm{C}(14)-\mathrm{C}(13)-\mathrm{H}(13)$ & 120.6 \\
\hline $\mathrm{H}(2 \mathrm{~A})-\mathrm{C}(2)-\mathrm{H}(2 \mathrm{C})$ & 109.5 & $\mathrm{C}(12)-\mathrm{C}(13)-\mathrm{H}(13)$ & 120.6 \\
\hline $\mathrm{H}(2 \mathrm{~B})-\mathrm{C}(2)-\mathrm{H}(2 \mathrm{C})$ & 109.5 & N(2)-C(14)-C(13) & $121.15(17)$ \\
\hline $\mathrm{N}(1)-\mathrm{C}(3)-\mathrm{C}(4)$ & $120.85(16)$ & $N(2)-C(14)-C(15)$ & $115.85(16)$ \\
\hline $\mathrm{N}(1)-\mathrm{C}(3)-\mathrm{C}(16)$ & $115.40(15)$ & $C(13)-C(14)-C(15)$ & $122.91(17)$ \\
\hline$C(4)-C(3)-C(16)$ & $123.69(15)$ & $\mathrm{N}(4)-\mathrm{C}(15)-\mathrm{C}(14)$ & $112.96(14)$ \\
\hline$C(3)-C(4)-C(5)$ & $118.80(16)$ & $\mathrm{N}(4)-\mathrm{C}(15)-\mathrm{H}(15 \mathrm{~A})$ & 109.0 \\
\hline $\mathrm{C}(3)-\mathrm{C}(4)-\mathrm{H}(4)$ & 120.6 & $\mathrm{C}(14)-\mathrm{C}(15)-\mathrm{H}(15 \mathrm{~A})$ & 109.0 \\
\hline $\mathrm{C}(5)-\mathrm{C}(4)-\mathrm{H}(4)$ & 120.6 & $\mathrm{~N}(4)-\mathrm{C}(15)-\mathrm{H}(15 \mathrm{~B})$ & 109.0 \\
\hline$C(6)-C(5)-C(4)$ & 119.61(17) & $\mathrm{C}(14)-\mathrm{C}(15)-\mathrm{H}(15 \mathrm{~B})$ & 109.0 \\
\hline $\mathrm{C}(6)-\mathrm{C}(5)-\mathrm{H}(5)$ & 120.2 & $\mathrm{H}(15 \mathrm{~A})-\mathrm{C}(15)-\mathrm{H}(15 \mathrm{~B})$ & 107.8 \\
\hline $\mathrm{C}(4)-\mathrm{C}(5)-\mathrm{H}(5)$ & 120.2 & $\mathrm{~N}(4)-\mathrm{C}(16)-\mathrm{C}(3)$ & $112.52(14)$ \\
\hline$C(5)-C(6)-C(7)$ & $118.94(16)$ & $\mathrm{N}(4)-\mathrm{C}(16)-\mathrm{H}(16 \mathrm{~A})$ & 109.1 \\
\hline $\mathrm{C}(5)-\mathrm{C}(6)-\mathrm{H}(6)$ & 120.5 & $\mathrm{C}(3)-\mathrm{C}(16)-\mathrm{H}(16 \mathrm{~A})$ & 109.1 \\
\hline $\mathrm{C}(7)-\mathrm{C}(6)-\mathrm{H}(6)$ & 120.5 & $\mathrm{~N}(4)-\mathrm{C}(16)-\mathrm{H}(16 \mathrm{~B})$ & 109.1 \\
\hline $\mathrm{N}(1)-\mathrm{C}(7)-\mathrm{C}(6)$ & $120.86(16)$ & $\mathrm{C}(3)-\mathrm{C}(16)-\mathrm{H}(16 \mathrm{~B})$ & 109.1 \\
\hline $\mathrm{N}(1)-\mathrm{C}(7)-\mathrm{C}(8)$ & $116.22(15)$ & $\mathrm{H}(16 \mathrm{~A})-\mathrm{C}(16)-\mathrm{H}(16 \mathrm{~B})$ & 107.8 \\
\hline$C(6)-C(7)-C(8)$ & $122.84(16)$ & $\mathrm{N}(3)-\mathrm{C}(17)-\mathrm{H}(17 \mathrm{~A})$ & 109.5 \\
\hline $\mathrm{N}(3)-\mathrm{C}(8)-\mathrm{C}(7)$ & $114.13(14)$ & $\mathrm{N}(3)-\mathrm{C}(17)-\mathrm{H}(17 \mathrm{~B})$ & 109.5 \\
\hline $\mathrm{N}(3)-\mathrm{C}(8)-\mathrm{H}(8 \mathrm{~A})$ & 108.7 & $\mathrm{H}(17 \mathrm{~A})-\mathrm{C}(17)-\mathrm{H}(17 \mathrm{~B})$ & 109.5 \\
\hline $\mathrm{C}(7)-\mathrm{C}(8)-\mathrm{H}(8 \mathrm{~A})$ & 108.7 & $\mathrm{~N}(3)-\mathrm{C}(17)-\mathrm{H}(17 \mathrm{C})$ & 109.5 \\
\hline N(3)-C(8)-H(8B) & 108.7 & $\mathrm{H}(17 \mathrm{~A})-\mathrm{C}(17)-\mathrm{H}(17 \mathrm{C})$ & 109.5 \\
\hline $\mathrm{C}(7)-\mathrm{C}(8)-\mathrm{H}(8 \mathrm{~B})$ & 108.7 & $\mathrm{H}(17 \mathrm{~B})-\mathrm{C}(17)-\mathrm{H}(17 \mathrm{C})$ & 109.5 \\
\hline $\mathrm{H}(8 \mathrm{~A})-\mathrm{C}(8)-\mathrm{H}(8 \mathrm{~B})$ & 107.6 & $\mathrm{~N}(4)-\mathrm{C}(18)-\mathrm{H}(18 \mathrm{~A})$ & 109.5 \\
\hline
\end{tabular}




\begin{tabular}{|c|c|c|c|}
\hline $\mathrm{N}(4)-\mathrm{C}(18)-\mathrm{H}(18 \mathrm{~B})$ & 109.5 & $\mathrm{C}(21)-\mathrm{C}(22)-\mathrm{H}(22)$ & 120.4 \\
\hline $\mathrm{H}(18 \mathrm{~A})-\mathrm{C}(18)-\mathrm{H}(18 \mathrm{~B})$ & 109.5 & $\mathrm{C}(23)-\mathrm{C}(22)-\mathrm{H}(22)$ & 120.4 \\
\hline $\mathrm{N}(4)-\mathrm{C}(18)-\mathrm{H}(18 \mathrm{C})$ & 109.5 & $C(22)-C(23)-C(24)$ & $120.03(19)$ \\
\hline $\mathrm{H}(18 \mathrm{~A})-\mathrm{C}(18)-\mathrm{H}(18 \mathrm{C})$ & 109.5 & $\mathrm{C}(22)-\mathrm{C}(23)-\mathrm{H}(23)$ & 120.0 \\
\hline $\mathrm{H}(18 \mathrm{~B})-\mathrm{C}(18)-\mathrm{H}(18 \mathrm{C})$ & 109.5 & $\mathrm{C}(24)-\mathrm{C}(23)-\mathrm{H}(23)$ & 120.0 \\
\hline $\mathrm{C}(1 \mathrm{~S})-\mathrm{O}(1 \mathrm{~S})-\mathrm{C}(4 \mathrm{~S})$ & $108.59(17)$ & $C(23)-C(24)-C(19)$ & $122.72(19)$ \\
\hline $\mathrm{O}(1 \mathrm{~S})-\mathrm{C}(1 \mathrm{~S})-\mathrm{C}(2 \mathrm{~S})$ & $107.74(19)$ & $\mathrm{C}(23)-\mathrm{C}(24)-\mathrm{H}(24)$ & 118.6 \\
\hline $\mathrm{O}(1 \mathrm{~S})-\mathrm{C}(1 \mathrm{~S})-\mathrm{H}(1 \mathrm{SA})$ & 110.2 & $\mathrm{C}(19)-\mathrm{C}(24)-\mathrm{H}(24)$ & 118.6 \\
\hline $\mathrm{C}(2 \mathrm{~S})-\mathrm{C}(1 \mathrm{~S})-\mathrm{H}(1 \mathrm{SA})$ & 110.2 & $C(30)-C(25)-C(26)$ & $115.08(16)$ \\
\hline $\mathrm{O}(1 \mathrm{~S})-\mathrm{C}(1 \mathrm{~S})-\mathrm{H}(1 \mathrm{SB})$ & 110.2 & $\mathrm{C}(30)-\mathrm{C}(25)-\mathrm{B}(1)$ & $121.90(15)$ \\
\hline $\mathrm{C}(2 \mathrm{~S})-\mathrm{C}(1 \mathrm{~S})-\mathrm{H}(1 \mathrm{SB})$ & 110.2 & $\mathrm{C}(26)-\mathrm{C}(25)-\mathrm{B}(1)$ & $122.61(15)$ \\
\hline $\mathrm{H}(1 \mathrm{SA})-\mathrm{C}(1 \mathrm{~S})-\mathrm{H}(1 \mathrm{SB})$ & 108.5 & $C(27)-C(26)-C(25)$ & $122.76(17)$ \\
\hline$C(1 S)-C(2 S)-C(3 S)$ & $101.1(2)$ & $\mathrm{C}(27)-\mathrm{C}(26)-\mathrm{H}(26)$ & 118.6 \\
\hline $\mathrm{C}(1 \mathrm{~S})-\mathrm{C}(2 \mathrm{~S})-\mathrm{H}(2 \mathrm{SA})$ & 111.6 & $\mathrm{C}(25)-\mathrm{C}(26)-\mathrm{H}(26)$ & 118.6 \\
\hline $\mathrm{C}(3 \mathrm{~S})-\mathrm{C}(2 \mathrm{~S})-\mathrm{H}(2 \mathrm{SA})$ & 111.6 & $\mathrm{C}(28)-\mathrm{C}(27)-\mathrm{C}(26)$ & $120.27(17)$ \\
\hline $\mathrm{C}(1 \mathrm{~S})-\mathrm{C}(2 \mathrm{~S})-\mathrm{H}(2 \mathrm{SB})$ & 111.6 & $\mathrm{C}(28)-\mathrm{C}(27)-\mathrm{H}(27)$ & 119.9 \\
\hline $\mathrm{C}(3 \mathrm{~S})-\mathrm{C}(2 \mathrm{~S})-\mathrm{H}(2 \mathrm{SB})$ & 111.6 & $\mathrm{C}(26)-\mathrm{C}(27)-\mathrm{H}(27)$ & 119.9 \\
\hline $\mathrm{H}(2 \mathrm{SA})-\mathrm{C}(2 \mathrm{~S})-\mathrm{H}(2 \mathrm{SB})$ & 109.4 & $C(29)-C(28)-C(27)$ & $118.67(17)$ \\
\hline $\mathrm{C}(4 \mathrm{~S})-\mathrm{C}(3 \mathrm{~S})-\mathrm{C}(2 \mathrm{~S})$ & $102.12(19)$ & $\mathrm{C}(29)-\mathrm{C}(28)-\mathrm{H}(28)$ & 120.7 \\
\hline $\mathrm{C}(4 \mathrm{~S})-\mathrm{C}(3 \mathrm{~S})-\mathrm{H}(3 \mathrm{SA})$ & 111.3 & $\mathrm{C}(27)-\mathrm{C}(28)-\mathrm{H}(28)$ & 120.7 \\
\hline $\mathrm{C}(2 \mathrm{~S})-\mathrm{C}(3 \mathrm{~S})-\mathrm{H}(3 \mathrm{SA})$ & 111.3 & $C(28)-C(29)-C(30)$ & $120.36(17)$ \\
\hline $\mathrm{C}(4 \mathrm{~S})-\mathrm{C}(3 \mathrm{~S})-\mathrm{H}(3 \mathrm{SB})$ & 111.3 & $\mathrm{C}(28)-\mathrm{C}(29)-\mathrm{H}(29)$ & 119.8 \\
\hline $\mathrm{C}(2 \mathrm{~S})-\mathrm{C}(3 \mathrm{~S})-\mathrm{H}(3 \mathrm{SB})$ & 111.3 & $\mathrm{C}(30)-\mathrm{C}(29)-\mathrm{H}(29)$ & 119.8 \\
\hline $\mathrm{H}(3 \mathrm{SA})-\mathrm{C}(3 \mathrm{~S})-\mathrm{H}(3 \mathrm{SB})$ & 109.2 & $C(29)-C(30)-C(25)$ & $122.85(17)$ \\
\hline $\mathrm{O}(1 \mathrm{~S})-\mathrm{C}(4 \mathrm{~S})-\mathrm{C}(3 \mathrm{~S})$ & $105.58(19)$ & $\mathrm{C}(29)-\mathrm{C}(30)-\mathrm{H}(30)$ & 118.6 \\
\hline $\mathrm{O}(1 \mathrm{~S})-\mathrm{C}(4 \mathrm{~S})-\mathrm{H}(4 \mathrm{SA})$ & 110.6 & $\mathrm{C}(25)-\mathrm{C}(30)-\mathrm{H}(30)$ & 118.6 \\
\hline $\mathrm{C}(3 \mathrm{~S})-\mathrm{C}(4 \mathrm{~S})-\mathrm{H}(4 \mathrm{SA})$ & 110.6 & $C(36)-C(31)-C(32)$ & $115.30(16)$ \\
\hline $\mathrm{O}(1 \mathrm{~S})-\mathrm{C}(4 \mathrm{~S})-\mathrm{H}(4 \mathrm{SB})$ & 110.6 & $\mathrm{C}(36)-\mathrm{C}(31)-\mathrm{B}(1)$ & $122.82(16)$ \\
\hline $\mathrm{C}(3 \mathrm{~S})-\mathrm{C}(4 \mathrm{~S})-\mathrm{H}(4 \mathrm{SB})$ & 110.6 & $\mathrm{C}(32)-\mathrm{C}(31)-\mathrm{B}(1)$ & $121.59(16)$ \\
\hline $\mathrm{H}(4 \mathrm{SA})-\mathrm{C}(4 \mathrm{~S})-\mathrm{H}(4 \mathrm{SB})$ & 108.8 & $\mathrm{C}(33)-\mathrm{C}(32)-\mathrm{C}(31)$ & $122.94(18)$ \\
\hline$C(24)-C(19)-C(20)$ & $115.28(16)$ & $\mathrm{C}(33)-\mathrm{C}(32)-\mathrm{H}(32)$ & 118.5 \\
\hline $\mathrm{C}(24)-\mathrm{C}(19)-\mathrm{B}(1)$ & $123.96(16)$ & $\mathrm{C}(31)-\mathrm{C}(32)-\mathrm{H}(32)$ & 118.5 \\
\hline $\mathrm{C}(20)-\mathrm{C}(19)-\mathrm{B}(1)$ & $120.40(16)$ & $\mathrm{C}(34)-\mathrm{C}(33)-\mathrm{C}(32)$ & $119.62(18)$ \\
\hline$C(21)-C(20)-C(19)$ & $122.63(19)$ & $\mathrm{C}(34)-\mathrm{C}(33)-\mathrm{H}(33)$ & 120.2 \\
\hline $\mathrm{C}(21)-\mathrm{C}(20)-\mathrm{H}(20)$ & 118.7 & $\mathrm{C}(32)-\mathrm{C}(33)-\mathrm{H}(33)$ & 120.2 \\
\hline $\mathrm{C}(19)-\mathrm{C}(20)-\mathrm{H}(20)$ & 118.7 & $C(35)-C(34)-C(33)$ & $119.47(18)$ \\
\hline$C(22)-C(21)-C(20)$ & $120.08(19)$ & $\mathrm{C}(35)-\mathrm{C}(34)-\mathrm{H}(34)$ & 120.3 \\
\hline $\mathrm{C}(22)-\mathrm{C}(21)-\mathrm{H}(21)$ & 120.0 & $\mathrm{C}(33)-\mathrm{C}(34)-\mathrm{H}(34)$ & 120.3 \\
\hline $\mathrm{C}(20)-\mathrm{C}(21)-\mathrm{H}(21)$ & 120.0 & $C(34)-C(35)-C(36)$ & $120.13(19)$ \\
\hline$C(21)-C(22)-C(23)$ & $119.20(17)$ & $\mathrm{C}(34)-\mathrm{C}(35)-\mathrm{H}(35)$ & 119.9 \\
\hline
\end{tabular}




$\begin{array}{llll}\mathrm{C}(36)-\mathrm{C}(35)-\mathrm{H}(35) & 119.9 & \mathrm{C}(41)-\mathrm{C}(40)-\mathrm{H}(40) & 120.4 \\ \mathrm{C}(35)-\mathrm{C}(36)-\mathrm{C}(31) & 122.46(18) & \mathrm{C}(39)-\mathrm{C}(40)-\mathrm{H}(40) & 120.4 \\ \mathrm{C}(35)-\mathrm{C}(36)-\mathrm{H}(36) & 118.8 & \mathrm{C}(40)-\mathrm{C}(41)-\mathrm{C}(42) & 119.88(18) \\ \mathrm{C}(31)-\mathrm{C}(36)-\mathrm{H}(36) & 118.8 & \mathrm{C}(40)-\mathrm{C}(41)-\mathrm{H}(41) & 120.1 \\ \mathrm{C}(42)-\mathrm{C}(37)-\mathrm{C}(38) & 115.69(16) & \mathrm{C}(42)-\mathrm{C}(41)-\mathrm{H}(41) & 120.1 \\ \mathrm{C}(42)-\mathrm{C}(37)-\mathrm{B}(1) & 122.14(15) & \mathrm{C}(41)-\mathrm{C}(42)-\mathrm{C}(37) & 122.67(18) \\ \mathrm{C}(38)-\mathrm{C}(37)-\mathrm{B}(1) & 121.88(16) & \mathrm{C}(41)-\mathrm{C}(42)-\mathrm{H}(42) & 118.7 \\ \mathrm{C}(39)-\mathrm{C}(38)-\mathrm{C}(37) & 122.36(18) & \mathrm{C}(37)-\mathrm{C}(42)-\mathrm{H}(42) & 118.7 \\ \mathrm{C}(39)-\mathrm{C}(38)-\mathrm{H}(38) & 118.8 & \mathrm{C}(37)-\mathrm{B}(1)-\mathrm{C}(25) & 112.17(14) \\ \mathrm{C}(37)-\mathrm{C}(38)-\mathrm{H}(38) & 118.8 & \mathrm{C}(37)-\mathrm{B}(1)-\mathrm{C}(31) & 104.59(13) \\ \mathrm{C}(40)-\mathrm{C}(39)-\mathrm{C}(38) & 120.19(18) & \mathrm{C}(25)-\mathrm{B}(1)-\mathrm{C}(31) & 112.04(14) \\ \mathrm{C}(40)-\mathrm{C}(39)-\mathrm{H}(39) & 119.9 & \mathrm{C}(37)-\mathrm{B}(1)-\mathrm{C}(19) & 111.37(14) \\ \mathrm{C}(38)-\mathrm{C}(39)-\mathrm{H}(39) & 119.9 & \mathrm{C}(25)-\mathrm{B}(1)-\mathrm{C}(19) & 103.18(13) \\ \mathrm{C}(41)-\mathrm{C}(40)-\mathrm{C}(39) & 119.19(17) & \mathrm{C}(31)-\mathrm{B}(1)-\mathrm{C}(19) & 113.74(14)\end{array}$

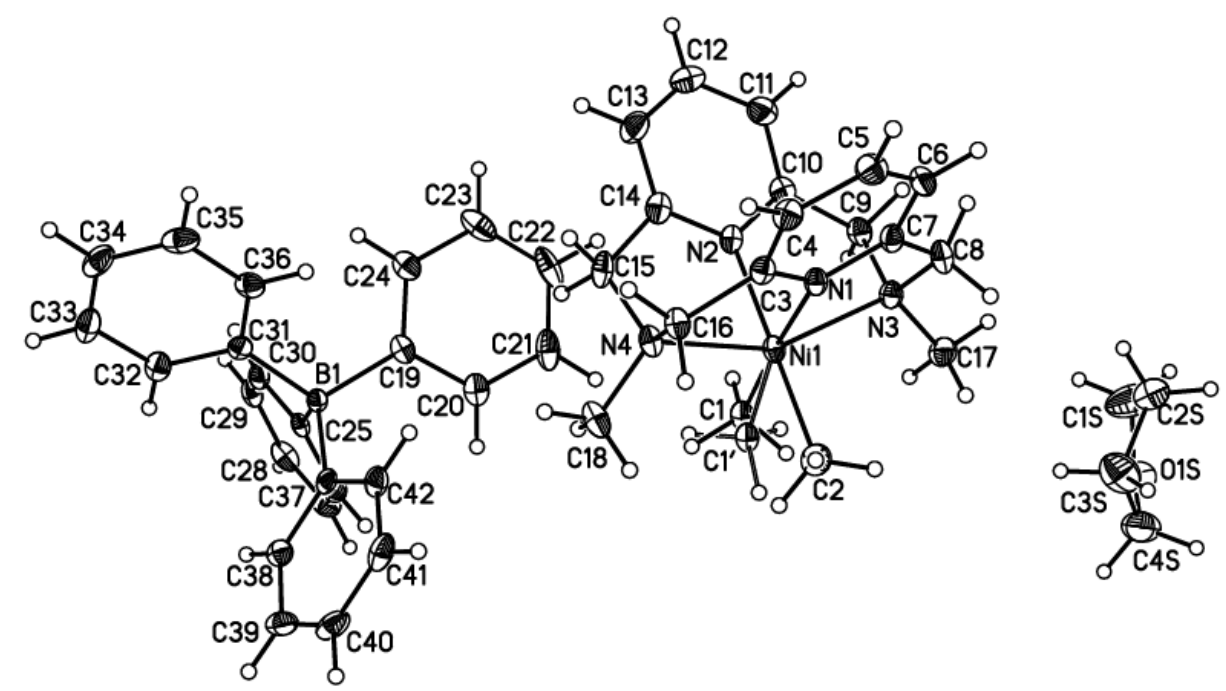

Figure S28. Projection view of $1 \mathrm{~m} 8714$ with 50\% thermal ellipsoids. 


\section{$\mathrm{X}$-ray structure determination of ( $\left.{ }^{\mathrm{Me}} \mathrm{N} 4\right) \mathrm{Ni}^{\mathrm{II}}$ (cycloneophyl)}

Table S23. Crystal data and structure refinement for $1 \mathrm{~m} 19415 \mathrm{t} 5$.

Identification code

Empirical formula

Formula weight

Temperature

Wavelength

Crystal system

Space group

Unit cell dimensions

Volume

Z

Density (calculated)

Absorption coefficient

$\mathrm{F}(000)$

Crystal size

Theta range for data collection

Index ranges

Reflections collected

Independent reflections

Completeness to theta $=25.242^{\circ}$

Absorption correction

Max. and min. transmission

Refinement method

Data / restraints / parameters

Goodness-of-fit on $\mathrm{F}^{2}$

Final $\mathrm{R}$ indices [I $>2 \operatorname{sigma}(\mathrm{I})]$

$\mathrm{R}$ indices (all data)

Absolute structure parameter

Largest diff. peak and hole
119415t5/lt/JS-092815-01

$\mathrm{C}_{30} \mathrm{H}_{40} \mathrm{~N}_{4} \mathrm{Ni} \mathrm{O}$

531.37

100(2) K

$0.71073 \AA$

Monoclinic

Pn

$\mathrm{a}=11.5021(9) \AA \quad \alpha=90^{\circ}$.

$\mathrm{b}=17.8327(13) \AA$

$\beta=90.026(5)^{\circ}$.

$\mathrm{c}=12.9451(11) \AA$

$\gamma=90^{\circ}$.

2655.2(4) $\AA^{3}$

4

$1.329 \mathrm{Mg} / \mathrm{m}^{3}$

$0.761 \mathrm{~mm}^{-1}$

1136

$0.589 \times 0.566 \times 0.232 \mathrm{~mm}^{3}$

1.142 to $30.624^{\circ}$.

$-16 \leq \mathrm{h} \leq 16,-25 \leq \mathrm{k} \leq 25,-18 \leq \mathrm{l} \leq 18$

29108

$29108[\mathrm{R}(\mathrm{int})=0.042]$

$99.5 \%$

Semi-empirical from equivalents

0.746070 and 0.621766

Full-matrix least-squares on $\mathrm{F}^{2}$

29108 / 2 / 658

1.041

$\mathrm{R} 1=0.0508, \mathrm{wR} 2=0.1253$

$\mathrm{R} 1=0.0587, \mathrm{wR} 2=0.1316$

$-0.010(16)$

1.273 and -1.586 e. $\AA^{-3}$ 
Table S24. Bond lengths $[\AA]$ and angles $\left[{ }^{\circ}\right]$ for $119415 t 5$.

\begin{tabular}{|c|c|c|c|}
\hline $\mathrm{Ni}(1)-\mathrm{C}(1)$ & $1.879(9)$ & $\mathrm{C}(10)-\mathrm{H}(10 \mathrm{~A})$ & 0.9800 \\
\hline $\mathrm{Ni}(1)-\mathrm{N}(1)$ & $1.936(7)$ & $\mathrm{C}(10)-\mathrm{H}(10 \mathrm{~B})$ & 0.9800 \\
\hline $\mathrm{Ni}(1)-\mathrm{C}(8)$ & $1.954(9)$ & $\mathrm{C}(10)-\mathrm{H}(10 \mathrm{C})$ & 0.9800 \\
\hline $\mathrm{Ni}(1)-\mathrm{N}(2)$ & $1.997(7)$ & $\mathrm{C}(11)-\mathrm{C}(12)$ & $1.366(13)$ \\
\hline $\mathrm{N}(1)-\mathrm{C}(15)$ & $1.339(10)$ & $C(11)-C(24)$ & $1.479(12)$ \\
\hline $\mathrm{N}(1)-\mathrm{C}(11)$ & $1.371(11)$ & $C(12)-C(13)$ & $1.373(13)$ \\
\hline $\mathrm{N}(2)-\mathrm{C}(18)$ & $1.343(11)$ & $\mathrm{C}(12)-\mathrm{H}(12)$ & 0.9500 \\
\hline $\mathrm{N}(2)-\mathrm{C}(22)$ & $1.367(12)$ & $C(13)-C(14)$ & $1.383(14)$ \\
\hline $\mathrm{N}(3)-\mathrm{C}(17)$ & $1.442(12)$ & $\mathrm{C}(13)-\mathrm{H}(13)$ & 0.9500 \\
\hline $\mathrm{N}(3)-\mathrm{C}(25)$ & $1.454(11)$ & $C(14)-C(15)$ & $1.374(12)$ \\
\hline $\mathrm{N}(3)-\mathrm{C}(16)$ & $1.473(11)$ & $\mathrm{C}(14)-\mathrm{H}(14)$ & 0.9500 \\
\hline $\mathrm{N}(4)-\mathrm{C}(23)$ & $1.448(12)$ & $C(15)-C(16)$ & $1.511(13)$ \\
\hline $\mathrm{N}(4)-\mathrm{C}(26)$ & $1.462(12)$ & $\mathrm{C}(16)-\mathrm{H}(16 \mathrm{~A})$ & 0.9900 \\
\hline $\mathrm{N}(4)-\mathrm{C}(24)$ & $1.476(11)$ & $\mathrm{C}(16)-\mathrm{H}(16 \mathrm{~B})$ & 0.9900 \\
\hline$C(1)-C(6)$ & $1.411(12)$ & $\mathrm{C}(17)-\mathrm{C}(18)$ & $1.524(12)$ \\
\hline$C(1)-C(2)$ & $1.421(12)$ & $\mathrm{C}(17)-\mathrm{H}(17 \mathrm{~A})$ & 0.9900 \\
\hline $\mathrm{C}(2)-\mathrm{C}(3)$ & $1.379(12)$ & $\mathrm{C}(17)-\mathrm{H}(17 \mathrm{~B})$ & 0.9900 \\
\hline $\mathrm{C}(2)-\mathrm{H}(2)$ & 0.9500 & $C(18)-C(19)$ & $1.393(12)$ \\
\hline$C(3)-C(4)$ & $1.391(13)$ & $C(19)-C(20)$ & $1.395(13)$ \\
\hline $\mathrm{C}(3)-\mathrm{H}(3)$ & 0.9500 & $\mathrm{C}(19)-\mathrm{H}(19)$ & 0.9500 \\
\hline $\mathrm{C}(4)-\mathrm{C}(5)$ & $1.391(13)$ & $C(20)-C(21)$ & $1.389(13)$ \\
\hline $\mathrm{C}(4)-\mathrm{H}(4 \mathrm{~A})$ & 0.9500 & $\mathrm{C}(20)-\mathrm{H}(20)$ & 0.9500 \\
\hline$C(5)-C(6)$ & $1.375(12)$ & $C(21)-C(22)$ & $1.389(13)$ \\
\hline $\mathrm{C}(5)-\mathrm{H}(5)$ & 0.9500 & $\mathrm{C}(21)-\mathrm{H}(21)$ & 0.9500 \\
\hline$C(6)-C(7)$ & $1.521(13)$ & $\mathrm{C}(22)-\mathrm{C}(23)$ & $1.515(13)$ \\
\hline$C(7)-C(8)$ & $1.506(12)$ & $\mathrm{C}(23)-\mathrm{H}(23 \mathrm{~A})$ & 0.9900 \\
\hline $\mathrm{C}(7)-\mathrm{C}(9)$ & $1.537(12)$ & $\mathrm{C}(23)-\mathrm{H}(23 \mathrm{~B})$ & 0.9900 \\
\hline$C(7)-C(10)$ & $1.546(13)$ & $\mathrm{C}(24)-\mathrm{H}(24 \mathrm{~A})$ & 0.9900 \\
\hline $\mathrm{C}(8)-\mathrm{H}(8 \mathrm{~A})$ & 0.9900 & $\mathrm{C}(24)-\mathrm{H}(24 \mathrm{~B})$ & 0.9900 \\
\hline $\mathrm{C}(8)-\mathrm{H}(8 \mathrm{~B})$ & 0.9900 & $\mathrm{C}(25)-\mathrm{H}(25 \mathrm{~A})$ & 0.9800 \\
\hline $\mathrm{C}(9)-\mathrm{H}(9 \mathrm{~A})$ & 0.9800 & $\mathrm{C}(25)-\mathrm{H}(25 \mathrm{~B})$ & 0.9800 \\
\hline C(9)-H(9B) & 0.9800 & $\mathrm{C}(25)-\mathrm{H}(25 \mathrm{C})$ & 0.9800 \\
\hline $\mathrm{C}(9)-\mathrm{H}(9 \mathrm{C})$ & 0.9800 & $\mathrm{C}(26)-\mathrm{H}(26 \mathrm{~A})$ & 0.9800 \\
\hline
\end{tabular}




\begin{tabular}{|c|c|c|c|}
\hline$C(26)-H(26 B)$ & 0.9800 & $\mathrm{C}(10 \mathrm{~A})-\mathrm{H}(10 \mathrm{E})$ & 0.9800 \\
\hline $\mathrm{C}(26)-\mathrm{H}(26 \mathrm{C})$ & 0.9800 & $\mathrm{C}(10 \mathrm{~A})-\mathrm{H}(10 \mathrm{~F})$ & 0.9800 \\
\hline $\mathrm{Ni}(1 \mathrm{~A})-\mathrm{C}(8 \mathrm{~A})$ & $1.903(8)$ & $C(11 \mathrm{~A})-\mathrm{C}(12 \mathrm{~A})$ & $1.405(13)$ \\
\hline $\mathrm{Ni}(1 \mathrm{~A})-\mathrm{C}(1 \mathrm{~A})$ & $1.905(9)$ & $\mathrm{C}(11 \mathrm{~A})-\mathrm{C}(24 \mathrm{~A})$ & $1.529(12)$ \\
\hline $\mathrm{Ni}(1 \mathrm{~A})-\mathrm{N}(2 \mathrm{~A})$ & $1.944(7)$ & $C(12 A)-C(13 A)$ & $1.386(13)$ \\
\hline $\mathrm{Ni}(1 \mathrm{~A})-\mathrm{N}(1 \mathrm{~A})$ & $1.973(7)$ & $\mathrm{C}(12 \mathrm{~A})-\mathrm{H}(12 \mathrm{~A})$ & 0.9500 \\
\hline$N(1 A)-C(15 A)$ & $1.350(11)$ & $C(13 A)-C(14 A)$ & $1.396(14)$ \\
\hline $\mathrm{N}(1 \mathrm{~A})-\mathrm{C}(11 \mathrm{~A})$ & $1.362(12)$ & $\mathrm{C}(13 \mathrm{~A})-\mathrm{H}(13 \mathrm{~A})$ & 0.9500 \\
\hline$N(2 A)-C(22 A)$ & $1.343(12)$ & $C(14 A)-C(15 A)$ & $1.401(13)$ \\
\hline $\mathrm{N}(2 \mathrm{~A})-\mathrm{C}(18 \mathrm{~A})$ & $1.350(11)$ & $\mathrm{C}(14 \mathrm{~A})-\mathrm{H}(14 \mathrm{~A})$ & 0.9500 \\
\hline $\mathrm{N}(3 \mathrm{~A})-\mathrm{C}(16 \mathrm{~A})$ & $1.453(10)$ & $C(15 A)-C(16 A)$ & $1.519(13)$ \\
\hline $\mathrm{N}(3 \mathrm{~A})-\mathrm{C}(25 \mathrm{~A})$ & $1.454(11)$ & $\mathrm{C}(16 \mathrm{~A})-\mathrm{H}(16 \mathrm{C})$ & 0.9900 \\
\hline $\mathrm{N}(3 \mathrm{~A})-\mathrm{C}(17 \mathrm{~A})$ & $1.465(11)$ & $\mathrm{C}(16 \mathrm{~A})-\mathrm{H}(16 \mathrm{D})$ & 0.9900 \\
\hline $\mathrm{N}(4 \mathrm{~A})-\mathrm{C}(24 \mathrm{~A})$ & $1.431(12)$ & $\mathrm{C}(17 \mathrm{~A})-\mathrm{C}(18 \mathrm{~A})$ & $1.515(13)$ \\
\hline $\mathrm{N}(4 \mathrm{~A})-\mathrm{C}(26 \mathrm{~A})$ & $1.451(11)$ & $\mathrm{C}(17 \mathrm{~A})-\mathrm{H}(17 \mathrm{C})$ & 0.9900 \\
\hline $\mathrm{N}(4 \mathrm{~A})-\mathrm{C}(23 \mathrm{~A})$ & $1.465(11)$ & $\mathrm{C}(17 \mathrm{~A})-\mathrm{H}(17 \mathrm{D})$ & 0.9900 \\
\hline$C(1 \mathrm{~A})-\mathrm{C}(2 \mathrm{~A})$ & $1.383(12)$ & $\mathrm{C}(18 \mathrm{~A})-\mathrm{C}(19 \mathrm{~A})$ & $1.370(11)$ \\
\hline$C(1 A)-C(6 A)$ & $1.409(11)$ & $C(19 A)-C(20 A)$ & $1.387(13)$ \\
\hline$C(2 A)-C(3 A)$ & $1.400(12)$ & $\mathrm{C}(19 \mathrm{~A})-\mathrm{H}(19 \mathrm{~A})$ & 0.9500 \\
\hline $\mathrm{C}(2 \mathrm{~A})-\mathrm{H}(2 \mathrm{~A})$ & 0.9500 & $C(20 A)-C(21 A)$ & $1.374(13)$ \\
\hline$C(3 A)-C(4 A)$ & $1.377(13)$ & $\mathrm{C}(20 \mathrm{~A})-\mathrm{H}(20 \mathrm{~A})$ & 0.9500 \\
\hline $\mathrm{C}(3 \mathrm{~A})-\mathrm{H}(3 \mathrm{AA})$ & 0.9500 & $\mathrm{C}(21 \mathrm{~A})-\mathrm{C}(22 \mathrm{~A})$ & $1.369(12)$ \\
\hline $\mathrm{C}(4 \mathrm{~A})-\mathrm{C}(5 \mathrm{~A})$ & $1.378(13)$ & $\mathrm{C}(21 \mathrm{~A})-\mathrm{H}(21 \mathrm{~A})$ & 0.9500 \\
\hline $\mathrm{C}(4 \mathrm{~A})-\mathrm{H}(4 \mathrm{AA})$ & 0.9500 & $C(22 A)-C(23 A)$ & $1.533(13)$ \\
\hline$C(5 A)-C(6 A)$ & $1.402(12)$ & $\mathrm{C}(23 \mathrm{~A})-\mathrm{H}(23 \mathrm{C})$ & 0.9900 \\
\hline $\mathrm{C}(5 \mathrm{~A})-\mathrm{H}(5 \mathrm{~A})$ & 0.9500 & $\mathrm{C}(23 \mathrm{~A})-\mathrm{H}(23 \mathrm{D})$ & 0.9900 \\
\hline$C(6 A)-C(7 A)$ & $1.490(12)$ & $\mathrm{C}(24 \mathrm{~A})-\mathrm{H}(24 \mathrm{C})$ & 0.9900 \\
\hline$C(7 A)-C(9 A)$ & $1.508(12)$ & $\mathrm{C}(24 \mathrm{~A})-\mathrm{H}(24 \mathrm{D})$ & 0.9900 \\
\hline$C(7 A)-C(8 A)$ & $1.554(13)$ & $\mathrm{C}(25 \mathrm{~A})-\mathrm{H}(25 \mathrm{D})$ & 0.9800 \\
\hline$C(7 A)-C(10 A)$ & $1.569(14)$ & $\mathrm{C}(25 \mathrm{~A})-\mathrm{H}(25 \mathrm{E})$ & 0.9800 \\
\hline $\mathrm{C}(8 \mathrm{~A})-\mathrm{H}(8 \mathrm{AA})$ & 0.9900 & $\mathrm{C}(25 \mathrm{~A})-\mathrm{H}(25 \mathrm{~F})$ & 0.9800 \\
\hline $\mathrm{C}(8 \mathrm{~A})-\mathrm{H}(8 \mathrm{AB})$ & 0.9900 & $\mathrm{C}(26 \mathrm{~A})-\mathrm{H}(26 \mathrm{D})$ & 0.9800 \\
\hline $\mathrm{C}(9 \mathrm{~A})-\mathrm{H}(9 \mathrm{AA})$ & 0.9800 & $\mathrm{C}(26 \mathrm{~A})-\mathrm{H}(26 \mathrm{E})$ & 0.9800 \\
\hline C(9A)-H(9AB) & 0.9800 & $\mathrm{C}(26 \mathrm{~A})-\mathrm{H}(26 \mathrm{~F})$ & 0.9800 \\
\hline $\mathrm{C}(9 \mathrm{~A})-\mathrm{H}(9 \mathrm{AC})$ & 0.9800 & $\mathrm{O}(1 \mathrm{~S})-\mathrm{C}(1 \mathrm{~S})$ & $1.409(13)$ \\
\hline $\mathrm{C}(10 \mathrm{~A})-\mathrm{H}(10 \mathrm{D})$ & 0.9800 & $\mathrm{O}(1 \mathrm{~S})-\mathrm{C}(4 \mathrm{~S})$ & $1.450(12)$ \\
\hline
\end{tabular}




\begin{tabular}{|c|c|c|c|}
\hline$C(1 S)-C(2 S)$ & $1.517(14)$ & $\mathrm{C}(17)-\mathrm{N}(3)-\mathrm{C}(25)$ & $113.4(7)$ \\
\hline C(1S)-H(1SA) & 0.9900 & $\mathrm{C}(17)-\mathrm{N}(3)-\mathrm{C}(16)$ & $118.1(6)$ \\
\hline C(1S)-H(1SB) & 0.9900 & $\mathrm{C}(25)-\mathrm{N}(3)-\mathrm{C}(16)$ & $115.0(7)$ \\
\hline $\mathrm{C}(2 \mathrm{~S})-\mathrm{C}(3 \mathrm{~S})$ & $1.500(16)$ & $\mathrm{C}(23)-\mathrm{N}(4)-\mathrm{C}(26)$ & $113.8(9)$ \\
\hline $\mathrm{C}(2 \mathrm{~S})-\mathrm{H}(2 \mathrm{SA})$ & 0.9900 & $\mathrm{C}(23)-\mathrm{N}(4)-\mathrm{C}(24)$ & $117.7(7)$ \\
\hline $\mathrm{C}(2 \mathrm{~S})-\mathrm{H}(2 \mathrm{SB})$ & 0.9900 & $\mathrm{C}(26)-\mathrm{N}(4)-\mathrm{C}(24)$ & $113.3(8)$ \\
\hline $\mathrm{C}(3 \mathrm{~S})-\mathrm{C}(4 \mathrm{~S})$ & $1.516(16)$ & $\mathrm{C}(6)-\mathrm{C}(1)-\mathrm{C}(2)$ & $117.8(8)$ \\
\hline C(3S)-H(3SA) & 0.9900 & $\mathrm{C}(6)-\mathrm{C}(1)-\mathrm{Ni}(1)$ & $116.6(7)$ \\
\hline C(3S)-H(3SB) & 0.9900 & $\mathrm{C}(2)-\mathrm{C}(1)-\mathrm{Ni}(1)$ & $125.6(6)$ \\
\hline C(4S)-H(4SA) & 0.9900 & $C(3)-C(2)-C(1)$ & $120.8(9)$ \\
\hline C(4S)-H(4SB) & 0.9900 & $\mathrm{C}(3)-\mathrm{C}(2)-\mathrm{H}(2)$ & 119.6 \\
\hline $\mathrm{O}(2 \mathrm{~S})-\mathrm{C}(8 \mathrm{~S})$ & $1.403(12)$ & $\mathrm{C}(1)-\mathrm{C}(2)-\mathrm{H}(2)$ & 119.6 \\
\hline $\mathrm{O}(2 \mathrm{~S})-\mathrm{C}(5 \mathrm{~S})$ & $1.426(14)$ & $\mathrm{C}(2)-\mathrm{C}(3)-\mathrm{C}(4)$ & $120.0(9)$ \\
\hline$C(5 S)-C(6 S)$ & $1.490(17)$ & $\mathrm{C}(2)-\mathrm{C}(3)-\mathrm{H}(3)$ & 120.0 \\
\hline $\mathrm{C}(5 \mathrm{~S})-\mathrm{H}(5 \mathrm{SA})$ & 0.9900 & $\mathrm{C}(4)-\mathrm{C}(3)-\mathrm{H}(3)$ & 120.0 \\
\hline $\mathrm{C}(5 \mathrm{~S})-\mathrm{H}(5 \mathrm{SB})$ & 0.9900 & $C(3)-C(4)-C(5)$ & $120.1(9)$ \\
\hline $\mathrm{C}(6 \mathrm{~S})-\mathrm{C}(7 \mathrm{~S})$ & $1.514(19)$ & $\mathrm{C}(3)-\mathrm{C}(4)-\mathrm{H}(4 \mathrm{~A})$ & 120.0 \\
\hline C(6S)-H(6SA) & 0.9900 & $\mathrm{C}(5)-\mathrm{C}(4)-\mathrm{H}(4 \mathrm{~A})$ & 120.0 \\
\hline $\mathrm{C}(6 \mathrm{~S})-\mathrm{H}(6 \mathrm{SB})$ & 0.9900 & $C(6)-C(5)-C(4)$ & $120.5(9)$ \\
\hline $\mathrm{C}(7 \mathrm{~S})-\mathrm{C}(8 \mathrm{~S})$ & $1.493(17)$ & $\mathrm{C}(6)-\mathrm{C}(5)-\mathrm{H}(5)$ & 119.7 \\
\hline C(7S)-H(7SA) & 0.9900 & $\mathrm{C}(4)-\mathrm{C}(5)-\mathrm{H}(5)$ & 119.7 \\
\hline C(7S)-H(7SB) & 0.9900 & $C(5)-C(6)-C(1)$ & $120.7(8)$ \\
\hline $\mathrm{C}(8 \mathrm{~S})-\mathrm{H}(8 \mathrm{~S} 1)$ & 0.9900 & $C(5)-C(6)-C(7)$ & $124.2(8)$ \\
\hline $\mathrm{C}(8 \mathrm{~S})-\mathrm{H}(8 \mathrm{~S} 2)$ & 0.9900 & $C(1)-C(6)-C(7)$ & $114.9(8)$ \\
\hline $\mathrm{C}(1)-\mathrm{Ni}(1)-\mathrm{N}(1)$ & $176.0(3)$ & $\mathrm{C}(8)-\mathrm{C}(7)-\mathrm{C}(6)$ & $105.3(7)$ \\
\hline C(1)-Ni(1)-C(8) & $83.5(3)$ & $\mathrm{C}(8)-\mathrm{C}(7)-\mathrm{C}(9)$ & $109.6(8)$ \\
\hline N(1)-Ni(1)-C(8) & $93.6(3)$ & $C(6)-C(7)-C(9)$ & $109.5(7)$ \\
\hline $\mathrm{C}(1)-\mathrm{Ni}(1)-\mathrm{N}(2)$ & $98.7(3)$ & $\mathrm{C}(8)-\mathrm{C}(7)-\mathrm{C}(10)$ & $111.4(8)$ \\
\hline $\mathrm{N}(1)-\mathrm{Ni}(1)-\mathrm{N}(2)$ & $84.4(3)$ & $C(6)-C(7)-C(10)$ & $112.5(8)$ \\
\hline $\mathrm{C}(8)-\mathrm{Ni}(1)-\mathrm{N}(2)$ & $175.3(4)$ & $\mathrm{C}(9)-\mathrm{C}(7)-\mathrm{C}(10)$ & $108.4(8)$ \\
\hline $\mathrm{C}(15)-\mathrm{N}(1)-\mathrm{C}(11)$ & $118.5(7)$ & $\mathrm{C}(7)-\mathrm{C}(8)-\mathrm{Ni}(1)$ & $113.8(6)$ \\
\hline $\mathrm{C}(15)-\mathrm{N}(1)-\mathrm{Ni}(1)$ & $120.2(6)$ & $\mathrm{C}(7)-\mathrm{C}(8)-\mathrm{H}(8 \mathrm{~A})$ & 108.8 \\
\hline $\mathrm{C}(11)-\mathrm{N}(1)-\mathrm{Ni}(1)$ & $120.8(6)$ & $\mathrm{Ni}(1)-\mathrm{C}(8)-\mathrm{H}(8 \mathrm{~A})$ & 108.8 \\
\hline $\mathrm{C}(18)-\mathrm{N}(2)-\mathrm{C}(22)$ & $117.0(8)$ & $\mathrm{C}(7)-\mathrm{C}(8)-\mathrm{H}(8 \mathrm{~B})$ & 108.8 \\
\hline $\mathrm{C}(18)-\mathrm{N}(2)-\mathrm{Ni}(1)$ & $121.9(6)$ & $\mathrm{Ni}(1)-\mathrm{C}(8)-\mathrm{H}(8 \mathrm{~B})$ & 108.8 \\
\hline $\mathrm{C}(22)-\mathrm{N}(2)-\mathrm{Ni}(1)$ & $120.1(6)$ & $\mathrm{H}(8 \mathrm{~A})-\mathrm{C}(8)-\mathrm{H}(8 \mathrm{~B})$ & 107.7 \\
\hline
\end{tabular}




\begin{tabular}{|c|c|c|c|}
\hline $\mathrm{C}(7)-\mathrm{C}(9)-\mathrm{H}(9 \mathrm{~A})$ & 109.5 & $\mathrm{~N}(3)-\mathrm{C}(17)-\mathrm{H}(17 \mathrm{~B})$ & 107.8 \\
\hline $\mathrm{C}(7)-\mathrm{C}(9)-\mathrm{H}(9 \mathrm{~B})$ & 109.5 & $\mathrm{C}(18)-\mathrm{C}(17)-\mathrm{H}(17 \mathrm{~B})$ & 107.8 \\
\hline $\mathrm{H}(9 \mathrm{~A})-\mathrm{C}(9)-\mathrm{H}(9 \mathrm{~B})$ & 109.5 & $\mathrm{H}(17 \mathrm{~A})-\mathrm{C}(17)-\mathrm{H}(17 \mathrm{~B})$ & 107.2 \\
\hline $\mathrm{C}(7)-\mathrm{C}(9)-\mathrm{H}(9 \mathrm{C})$ & 109.5 & $\mathrm{~N}(2)-\mathrm{C}(18)-\mathrm{C}(19)$ & $123.4(8)$ \\
\hline $\mathrm{H}(9 \mathrm{~A})-\mathrm{C}(9)-\mathrm{H}(9 \mathrm{C})$ & 109.5 & $\mathrm{~N}(2)-\mathrm{C}(18)-\mathrm{C}(17)$ & $114.6(8)$ \\
\hline H(9B)-C(9)-H(9C) & 109.5 & $\mathrm{C}(19)-\mathrm{C}(18)-\mathrm{C}(17)$ & $121.9(8)$ \\
\hline $\mathrm{C}(7)-\mathrm{C}(10)-\mathrm{H}(10 \mathrm{~A})$ & 109.5 & $\mathrm{C}(18)-\mathrm{C}(19)-\mathrm{C}(20)$ & $119.6(8)$ \\
\hline $\mathrm{C}(7)-\mathrm{C}(10)-\mathrm{H}(10 \mathrm{~B})$ & 109.5 & $\mathrm{C}(18)-\mathrm{C}(19)-\mathrm{H}(19)$ & 120.2 \\
\hline $\mathrm{H}(10 \mathrm{~A})-\mathrm{C}(10)-\mathrm{H}(10 \mathrm{~B})$ & 109.5 & $\mathrm{C}(20)-\mathrm{C}(19)-\mathrm{H}(19)$ & 120.2 \\
\hline $\mathrm{C}(7)-\mathrm{C}(10)-\mathrm{H}(10 \mathrm{C})$ & 109.5 & $\mathrm{C}(21)-\mathrm{C}(20)-\mathrm{C}(19)$ & $117.3(8)$ \\
\hline $\mathrm{H}(10 \mathrm{~A})-\mathrm{C}(10)-\mathrm{H}(10 \mathrm{C})$ & 109.5 & $\mathrm{C}(21)-\mathrm{C}(20)-\mathrm{H}(20)$ & 121.3 \\
\hline $\mathrm{H}(10 \mathrm{~B})-\mathrm{C}(10)-\mathrm{H}(10 \mathrm{C})$ & 109.5 & $\mathrm{C}(19)-\mathrm{C}(20)-\mathrm{H}(20)$ & 121.3 \\
\hline $\mathrm{C}(12)-\mathrm{C}(11)-\mathrm{N}(1)$ & $121.8(8)$ & $\mathrm{C}(22)-\mathrm{C}(21)-\mathrm{C}(20)$ & $120.3(9)$ \\
\hline$C(12)-C(11)-C(24)$ & $120.8(9)$ & $\mathrm{C}(22)-\mathrm{C}(21)-\mathrm{H}(21)$ & 119.8 \\
\hline $\mathrm{N}(1)-\mathrm{C}(11)-\mathrm{C}(24)$ & 117.3(8) & $\mathrm{C}(20)-\mathrm{C}(21)-\mathrm{H}(21)$ & 119.8 \\
\hline$C(11)-C(12)-C(13)$ & 119.6(9) & $\mathrm{N}(2)-\mathrm{C}(22)-\mathrm{C}(21)$ & $122.4(8)$ \\
\hline $\mathrm{C}(11)-\mathrm{C}(12)-\mathrm{H}(12)$ & 120.2 & $\mathrm{~N}(2)-\mathrm{C}(22)-\mathrm{C}(23)$ & $116.5(8)$ \\
\hline $\mathrm{C}(13)-\mathrm{C}(12)-\mathrm{H}(12)$ & 120.2 & $\mathrm{C}(21)-\mathrm{C}(22)-\mathrm{C}(23)$ & $121.1(8)$ \\
\hline$C(12)-C(13)-C(14)$ & 118.5(9) & $\mathrm{N}(4)-\mathrm{C}(23)-\mathrm{C}(22)$ & $119.8(8)$ \\
\hline $\mathrm{C}(12)-\mathrm{C}(13)-\mathrm{H}(13)$ & 120.8 & $\mathrm{~N}(4)-\mathrm{C}(23)-\mathrm{H}(23 \mathrm{~A})$ & 107.4 \\
\hline $\mathrm{C}(14)-\mathrm{C}(13)-\mathrm{H}(13)$ & 120.8 & $\mathrm{C}(22)-\mathrm{C}(23)-\mathrm{H}(23 \mathrm{~A})$ & 107.4 \\
\hline$C(15)-C(14)-C(13)$ & $120.3(9)$ & $\mathrm{N}(4)-\mathrm{C}(23)-\mathrm{H}(23 \mathrm{~B})$ & 107.4 \\
\hline$C(15)-C(14)-H(14)$ & 119.9 & $\mathrm{C}(22)-\mathrm{C}(23)-\mathrm{H}(23 \mathrm{~B})$ & 107.4 \\
\hline $\mathrm{C}(13)-\mathrm{C}(14)-\mathrm{H}(14)$ & 119.9 & $\mathrm{H}(23 \mathrm{~A})-\mathrm{C}(23)-\mathrm{H}(23 \mathrm{~B})$ & 106.9 \\
\hline $\mathrm{N}(1)-\mathrm{C}(15)-\mathrm{C}(14)$ & 121.3(9) & $\mathrm{N}(4)-\mathrm{C}(24)-\mathrm{C}(11)$ & $117.9(7)$ \\
\hline $\mathrm{N}(1)-\mathrm{C}(15)-\mathrm{C}(16)$ & $116.2(7)$ & $\mathrm{N}(4)-\mathrm{C}(24)-\mathrm{H}(24 \mathrm{~A})$ & 107.8 \\
\hline$C(14)-C(15)-C(16)$ & $122.5(8)$ & $\mathrm{C}(11)-\mathrm{C}(24)-\mathrm{H}(24 \mathrm{~A})$ & 107.8 \\
\hline $\mathrm{N}(3)-\mathrm{C}(16)-\mathrm{C}(15)$ & $117.9(7)$ & N(4)-C(24)-H(24B) & 107.8 \\
\hline $\mathrm{N}(3)-\mathrm{C}(16)-\mathrm{H}(16 \mathrm{~A})$ & 107.8 & $\mathrm{C}(11)-\mathrm{C}(24)-\mathrm{H}(24 \mathrm{~B})$ & 107.8 \\
\hline$C(15)-C(16)-H(16 A)$ & 107.8 & $\mathrm{H}(24 \mathrm{~A})-\mathrm{C}(24)-\mathrm{H}(24 \mathrm{~B})$ & 107.2 \\
\hline $\mathrm{N}(3)-\mathrm{C}(16)-\mathrm{H}(16 \mathrm{~B})$ & 107.8 & $\mathrm{~N}(3)-\mathrm{C}(25)-\mathrm{H}(25 \mathrm{~A})$ & 109.5 \\
\hline $\mathrm{C}(15)-\mathrm{C}(16)-\mathrm{H}(16 \mathrm{~B})$ & 107.8 & $\mathrm{~N}(3)-\mathrm{C}(25)-\mathrm{H}(25 \mathrm{~B})$ & 109.5 \\
\hline $\mathrm{H}(16 \mathrm{~A})-\mathrm{C}(16)-\mathrm{H}(16 \mathrm{~B})$ & 107.2 & $\mathrm{H}(25 \mathrm{~A})-\mathrm{C}(25)-\mathrm{H}(25 \mathrm{~B})$ & 109.5 \\
\hline $\mathrm{N}(3)-\mathrm{C}(17)-\mathrm{C}(18)$ & $117.9(7)$ & $\mathrm{N}(3)-\mathrm{C}(25)-\mathrm{H}(25 \mathrm{C})$ & 109.5 \\
\hline N(3)-C(17)-H(17A) & 107.8 & $\mathrm{H}(25 \mathrm{~A})-\mathrm{C}(25)-\mathrm{H}(25 \mathrm{C})$ & 109.5 \\
\hline $\mathrm{C}(18)-\mathrm{C}(17)-\mathrm{H}(17 \mathrm{~A})$ & 107.8 & $\mathrm{H}(25 \mathrm{~B})-\mathrm{C}(25)-\mathrm{H}(25 \mathrm{C})$ & 109.5 \\
\hline
\end{tabular}




\begin{tabular}{|c|c|c|c|}
\hline $\mathrm{N}(4)-\mathrm{C}(26)-\mathrm{H}(26 \mathrm{~A})$ & 109.5 & $C(4 A)-C(5 A)-C(6 A)$ & $120.8(8)$ \\
\hline $\mathrm{N}(4)-\mathrm{C}(26)-\mathrm{H}(26 \mathrm{~B})$ & 109.5 & $\mathrm{C}(4 \mathrm{~A})-\mathrm{C}(5 \mathrm{~A})-\mathrm{H}(5 \mathrm{~A})$ & 119.6 \\
\hline $\mathrm{H}(26 \mathrm{~A})-\mathrm{C}(26)-\mathrm{H}(26 \mathrm{~B})$ & 109.5 & $\mathrm{C}(6 \mathrm{~A})-\mathrm{C}(5 \mathrm{~A})-\mathrm{H}(5 \mathrm{~A})$ & 119.6 \\
\hline $\mathrm{N}(4)-\mathrm{C}(26)-\mathrm{H}(26 \mathrm{C})$ & 109.5 & $C(5 A)-C(6 A)-C(1 A)$ & $121.9(8)$ \\
\hline $\mathrm{H}(26 \mathrm{~A})-\mathrm{C}(26)-\mathrm{H}(26 \mathrm{C})$ & 109.5 & $C(5 A)-C(6 A)-C(7 A)$ & $123.7(8)$ \\
\hline $\mathrm{H}(26 \mathrm{~B})-\mathrm{C}(26)-\mathrm{H}(26 \mathrm{C})$ & 109.5 & $C(1 A)-C(6 A)-C(7 A)$ & $114.3(8)$ \\
\hline C(8A)-Ni(1A)-C(1A) & $83.9(4)$ & $C(6 A)-C(7 A)-C(9 A)$ & $112.4(8)$ \\
\hline $\mathrm{C}(8 \mathrm{~A})-\mathrm{Ni}(1 \mathrm{~A})-\mathrm{N}(2 \mathrm{~A})$ & $175.8(4)$ & $C(6 A)-C(7 A)-C(8 A)$ & $106.4(7)$ \\
\hline $\mathrm{C}(1 \mathrm{~A})-\mathrm{Ni}(1 \mathrm{~A})-\mathrm{N}(2 \mathrm{~A})$ & $98.3(3)$ & $C(9 A)-C(7 A)-C(8 A)$ & $112.6(8)$ \\
\hline $\mathrm{C}(8 \mathrm{~A})-\mathrm{Ni}(1 \mathrm{~A})-\mathrm{N}(1 \mathrm{~A})$ & $94.3(3)$ & $C(6 A)-C(7 A)-C(10 A)$ & $108.0(7)$ \\
\hline $\mathrm{C}(1 \mathrm{~A})-\mathrm{Ni}(1 \mathrm{~A})-\mathrm{N}(1 \mathrm{~A})$ & $176.1(3)$ & $\mathrm{C}(9 \mathrm{~A})-\mathrm{C}(7 \mathrm{~A})-\mathrm{C}(10 \mathrm{~A})$ & $109.3(8)$ \\
\hline $\mathrm{N}(2 \mathrm{~A})-\mathrm{Ni}(1 \mathrm{~A})-\mathrm{N}(1 \mathrm{~A})$ & $83.7(3)$ & $C(8 A)-C(7 A)-C(10 A)$ & $107.9(8)$ \\
\hline $\mathrm{C}(15 \mathrm{~A})-\mathrm{N}(1 \mathrm{~A})-\mathrm{C}(11 \mathrm{~A})$ & $119.7(8)$ & $\mathrm{C}(7 \mathrm{~A})-\mathrm{C}(8 \mathrm{~A})-\mathrm{Ni}(1 \mathrm{~A})$ & $113.7(6)$ \\
\hline C(15A)-N(1A)-Ni(1A) & $118.9(6)$ & $\mathrm{C}(7 \mathrm{~A})-\mathrm{C}(8 \mathrm{~A})-\mathrm{H}(8 \mathrm{AA})$ & 108.8 \\
\hline C(11A)-N(1A)-Ni(1A) & $120.8(6)$ & $\mathrm{Ni}(1 \mathrm{~A})-\mathrm{C}(8 \mathrm{~A})-\mathrm{H}(8 \mathrm{AA})$ & 108.8 \\
\hline $\mathrm{C}(22 \mathrm{~A})-\mathrm{N}(2 \mathrm{~A})-\mathrm{C}(18 \mathrm{~A})$ & 119.2(7) & $\mathrm{C}(7 \mathrm{~A})-\mathrm{C}(8 \mathrm{~A})-\mathrm{H}(8 \mathrm{AB})$ & 108.8 \\
\hline C(22A)-N(2A)-Ni(1A) & $118.8(6)$ & $\mathrm{Ni}(1 \mathrm{~A})-\mathrm{C}(8 \mathrm{~A})-\mathrm{H}(8 \mathrm{AB})$ & 108.8 \\
\hline C(18A)-N(2A)-Ni(1A) & $121.3(6)$ & $\mathrm{H}(8 \mathrm{AA})-\mathrm{C}(8 \mathrm{~A})-\mathrm{H}(8 \mathrm{AB})$ & 107.7 \\
\hline $\mathrm{C}(16 \mathrm{~A})-\mathrm{N}(3 \mathrm{~A})-\mathrm{C}(25 \mathrm{~A})$ & $113.5(7)$ & $\mathrm{C}(7 \mathrm{~A})-\mathrm{C}(9 \mathrm{~A})-\mathrm{H}(9 \mathrm{AA})$ & 109.5 \\
\hline$C(16 A)-N(3 A)-C(17 A)$ & $116.8(7)$ & $\mathrm{C}(7 \mathrm{~A})-\mathrm{C}(9 \mathrm{~A})-\mathrm{H}(9 \mathrm{AB})$ & 109.5 \\
\hline $\mathrm{C}(25 \mathrm{~A})-\mathrm{N}(3 \mathrm{~A})-\mathrm{C}(17 \mathrm{~A})$ & $115.6(7)$ & H(9AA)-C(9A)-H(9AB) & 109.5 \\
\hline $\mathrm{C}(24 \mathrm{~A})-\mathrm{N}(4 \mathrm{~A})-\mathrm{C}(26 \mathrm{~A})$ & $113.2(7)$ & $\mathrm{C}(7 \mathrm{~A})-\mathrm{C}(9 \mathrm{~A})-\mathrm{H}(9 \mathrm{AC})$ & 109.5 \\
\hline $\mathrm{C}(24 \mathrm{~A})-\mathrm{N}(4 \mathrm{~A})-\mathrm{C}(23 \mathrm{~A})$ & $118.3(7)$ & H(9AA)-C(9A)-H(9AC) & 109.5 \\
\hline$C(26 A)-N(4 A)-C(23 A)$ & $115.0(8)$ & $H(9 A B)-C(9 A)-H(9 A C)$ & 109.5 \\
\hline$C(2 A)-C(1 A)-C(6 A)$ & $115.6(8)$ & $C(7 A)-C(10 A)-H(10 D)$ & 109.5 \\
\hline$C(2 A)-C(1 A)-N i(1 A)$ & $127.6(6)$ & $\mathrm{C}(7 \mathrm{~A})-\mathrm{C}(10 \mathrm{~A})-\mathrm{H}(10 \mathrm{E})$ & 109.5 \\
\hline$C(6 A)-C(1 A)-N i(1 A)$ & $116.7(6)$ & $H(10 D)-C(10 A)-H(10 E)$ & 109.5 \\
\hline$C(1 \mathrm{~A})-\mathrm{C}(2 \mathrm{~A})-\mathrm{C}(3 \mathrm{~A})$ & $122.5(8)$ & $\mathrm{C}(7 \mathrm{~A})-\mathrm{C}(10 \mathrm{~A})-\mathrm{H}(10 \mathrm{~F})$ & 109.5 \\
\hline$C(1 \mathrm{~A})-\mathrm{C}(2 \mathrm{~A})-\mathrm{H}(2 \mathrm{~A})$ & 118.7 & $\mathrm{H}(10 \mathrm{D})-\mathrm{C}(10 \mathrm{~A})-\mathrm{H}(10 \mathrm{~F})$ & 109.5 \\
\hline$C(3 A)-C(2 A)-H(2 A)$ & 118.7 & $\mathrm{H}(10 \mathrm{E})-\mathrm{C}(10 \mathrm{~A})-\mathrm{H}(10 \mathrm{~F})$ & 109.5 \\
\hline$C(4 A)-C(3 A)-C(2 A)$ & $120.9(8)$ & $\mathrm{N}(1 \mathrm{~A})-\mathrm{C}(11 \mathrm{~A})-\mathrm{C}(12 \mathrm{~A})$ & $119.9(8)$ \\
\hline $\mathrm{C}(4 \mathrm{~A})-\mathrm{C}(3 \mathrm{~A})-\mathrm{H}(3 \mathrm{AA})$ & 119.5 & $\mathrm{~N}(1 \mathrm{~A})-\mathrm{C}(11 \mathrm{~A})-\mathrm{C}(24 \mathrm{~A})$ & $117.6(8)$ \\
\hline $\mathrm{C}(2 \mathrm{~A})-\mathrm{C}(3 \mathrm{~A})-\mathrm{H}(3 \mathrm{AA})$ & 119.5 & $\mathrm{C}(12 \mathrm{~A})-\mathrm{C}(11 \mathrm{~A})-\mathrm{C}(24 \mathrm{~A})$ & $122.5(8)$ \\
\hline$C(3 A)-C(4 A)-C(5 A)$ & $118.2(9)$ & $C(13 A)-C(12 A)-C(11 A)$ & $121.4(9)$ \\
\hline $\mathrm{C}(3 \mathrm{~A})-\mathrm{C}(4 \mathrm{~A})-\mathrm{H}(4 \mathrm{AA})$ & 120.9 & $\mathrm{C}(13 \mathrm{~A})-\mathrm{C}(12 \mathrm{~A})-\mathrm{H}(12 \mathrm{~A})$ & 119.3 \\
\hline $\mathrm{C}(5 \mathrm{~A})-\mathrm{C}(4 \mathrm{~A})-\mathrm{H}(4 \mathrm{AA})$ & 120.9 & $\mathrm{C}(11 \mathrm{~A})-\mathrm{C}(12 \mathrm{~A})-\mathrm{H}(12 \mathrm{~A})$ & 119.3 \\
\hline
\end{tabular}




\begin{tabular}{|c|c|c|c|}
\hline $\mathrm{C}(12 \mathrm{~A})-\mathrm{C}(13 \mathrm{~A})-\mathrm{C}(14 \mathrm{~A})$ & $117.5(8)$ & $\mathrm{N}(4 \mathrm{~A})-\mathrm{C}(23 \mathrm{~A})-\mathrm{C}(22 \mathrm{~A})$ & $117.2(8)$ \\
\hline $\mathrm{C}(12 \mathrm{~A})-\mathrm{C}(13 \mathrm{~A})-\mathrm{H}(13 \mathrm{~A})$ & 121.3 & $\mathrm{~N}(4 \mathrm{~A})-\mathrm{C}(23 \mathrm{~A})-\mathrm{H}(23 \mathrm{C})$ & 108.0 \\
\hline $\mathrm{C}(14 \mathrm{~A})-\mathrm{C}(13 \mathrm{~A})-\mathrm{H}(13 \mathrm{~A})$ & 121.3 & $\mathrm{C}(22 \mathrm{~A})-\mathrm{C}(23 \mathrm{~A})-\mathrm{H}(23 \mathrm{C})$ & 108.0 \\
\hline$C(13 \mathrm{~A})-\mathrm{C}(14 \mathrm{~A})-\mathrm{C}(15 \mathrm{~A})$ & $119.8(8)$ & $\mathrm{N}(4 \mathrm{~A})-\mathrm{C}(23 \mathrm{~A})-\mathrm{H}(23 \mathrm{D})$ & 108.0 \\
\hline $\mathrm{C}(13 \mathrm{~A})-\mathrm{C}(14 \mathrm{~A})-\mathrm{H}(14 \mathrm{~A})$ & 120.1 & $\mathrm{C}(22 \mathrm{~A})-\mathrm{C}(23 \mathrm{~A})-\mathrm{H}(23 \mathrm{D})$ & 108.0 \\
\hline $\mathrm{C}(15 \mathrm{~A})-\mathrm{C}(14 \mathrm{~A})-\mathrm{H}(14 \mathrm{~A})$ & 120.1 & $\mathrm{H}(23 \mathrm{C})-\mathrm{C}(23 \mathrm{~A})-\mathrm{H}(23 \mathrm{D})$ & 107.2 \\
\hline $\mathrm{N}(1 \mathrm{~A})-\mathrm{C}(15 \mathrm{~A})-\mathrm{C}(14 \mathrm{~A})$ & $121.7(9)$ & $\mathrm{N}(4 \mathrm{~A})-\mathrm{C}(24 \mathrm{~A})-\mathrm{C}(11 \mathrm{~A})$ & $118.4(7)$ \\
\hline $\mathrm{N}(1 \mathrm{~A})-\mathrm{C}(15 \mathrm{~A})-\mathrm{C}(16 \mathrm{~A})$ & $118.1(8)$ & $\mathrm{N}(4 \mathrm{~A})-\mathrm{C}(24 \mathrm{~A})-\mathrm{H}(24 \mathrm{C})$ & 107.7 \\
\hline$C(14 A)-C(15 A)-C(16 A)$ & $120.2(8)$ & $\mathrm{C}(11 \mathrm{~A})-\mathrm{C}(24 \mathrm{~A})-\mathrm{H}(24 \mathrm{C})$ & 107.7 \\
\hline $\mathrm{N}(3 \mathrm{~A})-\mathrm{C}(16 \mathrm{~A})-\mathrm{C}(15 \mathrm{~A})$ & $118.5(7)$ & $\mathrm{N}(4 \mathrm{~A})-\mathrm{C}(24 \mathrm{~A})-\mathrm{H}(24 \mathrm{D})$ & 107.7 \\
\hline $\mathrm{N}(3 \mathrm{~A})-\mathrm{C}(16 \mathrm{~A})-\mathrm{H}(16 \mathrm{C})$ & 107.7 & $\mathrm{C}(11 \mathrm{~A})-\mathrm{C}(24 \mathrm{~A})-\mathrm{H}(24 \mathrm{D})$ & 107.7 \\
\hline $\mathrm{C}(15 \mathrm{~A})-\mathrm{C}(16 \mathrm{~A})-\mathrm{H}(16 \mathrm{C})$ & 107.7 & $\mathrm{H}(24 \mathrm{C})-\mathrm{C}(24 \mathrm{~A})-\mathrm{H}(24 \mathrm{D})$ & 107.1 \\
\hline $\mathrm{N}(3 \mathrm{~A})-\mathrm{C}(16 \mathrm{~A})-\mathrm{H}(16 \mathrm{D})$ & 107.7 & $\mathrm{~N}(3 \mathrm{~A})-\mathrm{C}(25 \mathrm{~A})-\mathrm{H}(25 \mathrm{D})$ & 109.5 \\
\hline $\mathrm{C}(15 \mathrm{~A})-\mathrm{C}(16 \mathrm{~A})-\mathrm{H}(16 \mathrm{D})$ & 107.7 & $\mathrm{~N}(3 \mathrm{~A})-\mathrm{C}(25 \mathrm{~A})-\mathrm{H}(25 \mathrm{E})$ & 109.5 \\
\hline $\mathrm{H}(16 \mathrm{C})-\mathrm{C}(16 \mathrm{~A})-\mathrm{H}(16 \mathrm{D})$ & 107.1 & $\mathrm{H}(25 \mathrm{D})-\mathrm{C}(25 \mathrm{~A})-\mathrm{H}(25 \mathrm{E})$ & 109.5 \\
\hline $\mathrm{N}(3 \mathrm{~A})-\mathrm{C}(17 \mathrm{~A})-\mathrm{C}(18 \mathrm{~A})$ & $117.7(8)$ & $\mathrm{N}(3 \mathrm{~A})-\mathrm{C}(25 \mathrm{~A})-\mathrm{H}(25 \mathrm{~F})$ & 109.5 \\
\hline $\mathrm{N}(3 \mathrm{~A})-\mathrm{C}(17 \mathrm{~A})-\mathrm{H}(17 \mathrm{C})$ & 107.9 & $\mathrm{H}(25 \mathrm{D})-\mathrm{C}(25 \mathrm{~A})-\mathrm{H}(25 \mathrm{~F})$ & 109.5 \\
\hline $\mathrm{C}(18 \mathrm{~A})-\mathrm{C}(17 \mathrm{~A})-\mathrm{H}(17 \mathrm{C})$ & 107.9 & $\mathrm{H}(25 \mathrm{E})-\mathrm{C}(25 \mathrm{~A})-\mathrm{H}(25 \mathrm{~F})$ & 109.5 \\
\hline $\mathrm{N}(3 \mathrm{~A})-\mathrm{C}(17 \mathrm{~A})-\mathrm{H}(17 \mathrm{D})$ & 107.9 & $\mathrm{~N}(4 \mathrm{~A})-\mathrm{C}(26 \mathrm{~A})-\mathrm{H}(26 \mathrm{D})$ & 109.5 \\
\hline $\mathrm{C}(18 \mathrm{~A})-\mathrm{C}(17 \mathrm{~A})-\mathrm{H}(17 \mathrm{D})$ & 107.9 & $\mathrm{~N}(4 \mathrm{~A})-\mathrm{C}(26 \mathrm{~A})-\mathrm{H}(26 \mathrm{E})$ & 109.5 \\
\hline $\mathrm{H}(17 \mathrm{C})-\mathrm{C}(17 \mathrm{~A})-\mathrm{H}(17 \mathrm{D})$ & 107.2 & $H(26 D)-C(26 A)-H(26 E)$ & 109.5 \\
\hline $\mathrm{N}(2 \mathrm{~A})-\mathrm{C}(18 \mathrm{~A})-\mathrm{C}(19 \mathrm{~A})$ & $121.9(8)$ & $\mathrm{N}(4 \mathrm{~A})-\mathrm{C}(26 \mathrm{~A})-\mathrm{H}(26 \mathrm{~F})$ & 109.5 \\
\hline $\mathrm{N}(2 \mathrm{~A})-\mathrm{C}(18 \mathrm{~A})-\mathrm{C}(17 \mathrm{~A})$ & $117.2(7)$ & $\mathrm{H}(26 \mathrm{D})-\mathrm{C}(26 \mathrm{~A})-\mathrm{H}(26 \mathrm{~F})$ & 109.5 \\
\hline $\mathrm{C}(19 \mathrm{~A})-\mathrm{C}(18 \mathrm{~A})-\mathrm{C}(17 \mathrm{~A})$ & $120.8(8)$ & $\mathrm{H}(26 \mathrm{E})-\mathrm{C}(26 \mathrm{~A})-\mathrm{H}(26 \mathrm{~F})$ & 109.5 \\
\hline$C(18 A)-C(19 A)-C(20 A)$ & $118.6(8)$ & $\mathrm{C}(1 \mathrm{~S})-\mathrm{O}(1 \mathrm{~S})-\mathrm{C}(4 \mathrm{~S})$ & $108.4(9)$ \\
\hline $\mathrm{C}(18 \mathrm{~A})-\mathrm{C}(19 \mathrm{~A})-\mathrm{H}(19 \mathrm{~A})$ & 120.7 & $\mathrm{O}(1 \mathrm{~S})-\mathrm{C}(1 \mathrm{~S})-\mathrm{C}(2 \mathrm{~S})$ & $108.0(8)$ \\
\hline$C(20 A)-C(19 A)-H(19 A)$ & 120.7 & $\mathrm{O}(1 \mathrm{~S})-\mathrm{C}(1 \mathrm{~S})-\mathrm{H}(1 \mathrm{SA})$ & 110.1 \\
\hline$C(21 A)-C(20 A)-C(19 A)$ & $119.1(8)$ & $\mathrm{C}(2 \mathrm{~S})-\mathrm{C}(1 \mathrm{~S})-\mathrm{H}(1 \mathrm{SA})$ & 110.1 \\
\hline$C(21 A)-C(20 A)-H(20 A)$ & 120.5 & $\mathrm{O}(1 \mathrm{~S})-\mathrm{C}(1 \mathrm{~S})-\mathrm{H}(1 \mathrm{SB})$ & 110.1 \\
\hline$C(19 A)-C(20 A)-H(20 A)$ & 120.5 & $\mathrm{C}(2 \mathrm{~S})-\mathrm{C}(1 \mathrm{~S})-\mathrm{H}(1 \mathrm{SB})$ & 110.1 \\
\hline$C(22 A)-C(21 A)-C(20 A)$ & $119.8(9)$ & $\mathrm{H}(1 \mathrm{SA})-\mathrm{C}(1 \mathrm{~S})-\mathrm{H}(1 \mathrm{SB})$ & 108.4 \\
\hline $\mathrm{C}(22 \mathrm{~A})-\mathrm{C}(21 \mathrm{~A})-\mathrm{H}(21 \mathrm{~A})$ & 120.1 & $\mathrm{C}(3 \mathrm{~S})-\mathrm{C}(2 \mathrm{~S})-\mathrm{C}(1 \mathrm{~S})$ & $102.6(9)$ \\
\hline $\mathrm{C}(20 \mathrm{~A})-\mathrm{C}(21 \mathrm{~A})-\mathrm{H}(21 \mathrm{~A})$ & 120.1 & $\mathrm{C}(3 \mathrm{~S})-\mathrm{C}(2 \mathrm{~S})-\mathrm{H}(2 \mathrm{SA})$ & 111.3 \\
\hline $\mathrm{N}(2 \mathrm{~A})-\mathrm{C}(22 \mathrm{~A})-\mathrm{C}(21 \mathrm{~A})$ & $121.2(9)$ & $\mathrm{C}(1 \mathrm{~S})-\mathrm{C}(2 \mathrm{~S})-\mathrm{H}(2 \mathrm{SA})$ & 111.3 \\
\hline $\mathrm{N}(2 \mathrm{~A})-\mathrm{C}(22 \mathrm{~A})-\mathrm{C}(23 \mathrm{~A})$ & $118.0(8)$ & $\mathrm{C}(3 \mathrm{~S})-\mathrm{C}(2 \mathrm{~S})-\mathrm{H}(2 \mathrm{SB})$ & 111.3 \\
\hline$C(21 A)-C(22 A)-C(23 A)$ & $120.6(8)$ & $\mathrm{C}(1 \mathrm{~S})-\mathrm{C}(2 \mathrm{~S})-\mathrm{H}(2 \mathrm{SB})$ & 111.3 \\
\hline
\end{tabular}




\begin{tabular}{|c|c|c|c|}
\hline $\mathrm{H}(2 \mathrm{SA})-\mathrm{C}(2 \mathrm{~S})-\mathrm{H}(2 \mathrm{SB})$ & 109.2 & $\mathrm{H}(5 \mathrm{SA})-\mathrm{C}(5 \mathrm{~S})-\mathrm{H}(5 \mathrm{SB})$ & 108.4 \\
\hline $\mathrm{C}(2 \mathrm{~S})-\mathrm{C}(3 \mathrm{~S})-\mathrm{C}(4 \mathrm{~S})$ & $102.0(9)$ & $\mathrm{C}(5 \mathrm{~S})-\mathrm{C}(6 \mathrm{~S})-\mathrm{C}(7 \mathrm{~S})$ & $103.9(10)$ \\
\hline $\mathrm{C}(2 \mathrm{~S})-\mathrm{C}(3 \mathrm{~S})-\mathrm{H}(3 \mathrm{SA})$ & 111.4 & $\mathrm{C}(5 \mathrm{~S})-\mathrm{C}(6 \mathrm{~S})-\mathrm{H}(6 \mathrm{SA})$ & 111.0 \\
\hline $\mathrm{C}(4 \mathrm{~S})-\mathrm{C}(3 \mathrm{~S})-\mathrm{H}(3 \mathrm{SA})$ & 111.4 & $\mathrm{C}(7 \mathrm{~S})-\mathrm{C}(6 \mathrm{~S})-\mathrm{H}(6 \mathrm{SA})$ & 111.0 \\
\hline $\mathrm{C}(2 \mathrm{~S})-\mathrm{C}(3 \mathrm{~S})-\mathrm{H}(3 \mathrm{SB})$ & 111.4 & $\mathrm{C}(5 \mathrm{~S})-\mathrm{C}(6 \mathrm{~S})-\mathrm{H}(6 \mathrm{SB})$ & 111.0 \\
\hline $\mathrm{C}(4 \mathrm{~S})-\mathrm{C}(3 \mathrm{~S})-\mathrm{H}(3 \mathrm{SB})$ & 111.4 & $\mathrm{C}(7 \mathrm{~S})-\mathrm{C}(6 \mathrm{~S})-\mathrm{H}(6 \mathrm{SB})$ & 111.0 \\
\hline $\mathrm{H}(3 \mathrm{SA})-\mathrm{C}(3 \mathrm{~S})-\mathrm{H}(3 \mathrm{SB})$ & 109.2 & $\mathrm{H}(6 \mathrm{SA})-\mathrm{C}(6 \mathrm{~S})-\mathrm{H}(6 \mathrm{SB})$ & 109.0 \\
\hline $\mathrm{O}(1 \mathrm{~S})-\mathrm{C}(4 \mathrm{~S})-\mathrm{C}(3 \mathrm{~S})$ & $106.4(9)$ & $\mathrm{C}(8 \mathrm{~S})-\mathrm{C}(7 \mathrm{~S})-\mathrm{C}(6 \mathrm{~S})$ & $102.8(10)$ \\
\hline $\mathrm{O}(1 \mathrm{~S})-\mathrm{C}(4 \mathrm{~S})-\mathrm{H}(4 \mathrm{SA})$ & 110.4 & $\mathrm{C}(8 \mathrm{~S})-\mathrm{C}(7 \mathrm{~S})-\mathrm{H}(7 \mathrm{SA})$ & 111.2 \\
\hline $\mathrm{C}(3 \mathrm{~S})-\mathrm{C}(4 \mathrm{~S})-\mathrm{H}(4 \mathrm{SA})$ & 110.4 & $\mathrm{C}(6 \mathrm{~S})-\mathrm{C}(7 \mathrm{~S})-\mathrm{H}(7 \mathrm{SA})$ & 111.2 \\
\hline $\mathrm{O}(1 \mathrm{~S})-\mathrm{C}(4 \mathrm{~S})-\mathrm{H}(4 \mathrm{SB})$ & 110.4 & $\mathrm{C}(8 \mathrm{~S})-\mathrm{C}(7 \mathrm{~S})-\mathrm{H}(7 \mathrm{SB})$ & 111.2 \\
\hline $\mathrm{C}(3 \mathrm{~S})-\mathrm{C}(4 \mathrm{~S})-\mathrm{H}(4 \mathrm{SB})$ & 110.4 & $\mathrm{C}(6 \mathrm{~S})-\mathrm{C}(7 \mathrm{~S})-\mathrm{H}(7 \mathrm{SB})$ & 111.2 \\
\hline $\mathrm{H}(4 \mathrm{SA})-\mathrm{C}(4 \mathrm{~S})-\mathrm{H}(4 \mathrm{SB})$ & 108.6 & $\mathrm{H}(7 \mathrm{SA})-\mathrm{C}(7 \mathrm{~S})-\mathrm{H}(7 \mathrm{SB})$ & 109.1 \\
\hline $\mathrm{C}(8 \mathrm{~S})-\mathrm{O}(2 \mathrm{~S})-\mathrm{C}(5 \mathrm{~S})$ & $108.0(8)$ & $\mathrm{O}(2 \mathrm{~S})-\mathrm{C}(8 \mathrm{~S})-\mathrm{C}(7 \mathrm{~S})$ & $105.1(10)$ \\
\hline $\mathrm{O}(2 \mathrm{~S})-\mathrm{C}(5 \mathrm{~S})-\mathrm{C}(6 \mathrm{~S})$ & $107.9(9)$ & $\mathrm{O}(2 \mathrm{~S})-\mathrm{C}(8 \mathrm{~S})-\mathrm{H}(8 \mathrm{~S} 1)$ & 110.7 \\
\hline $\mathrm{O}(2 \mathrm{~S})-\mathrm{C}(5 \mathrm{~S})-\mathrm{H}(5 \mathrm{SA})$ & 110.1 & $\mathrm{C}(7 \mathrm{~S})-\mathrm{C}(8 \mathrm{~S})-\mathrm{H}(8 \mathrm{~S} 1)$ & 110.7 \\
\hline $\mathrm{C}(6 \mathrm{~S})-\mathrm{C}(5 \mathrm{~S})-\mathrm{H}(5 \mathrm{SA})$ & 110.1 & $\mathrm{O}(2 \mathrm{~S})-\mathrm{C}(8 \mathrm{~S})-\mathrm{H}(8 \mathrm{~S} 2)$ & 110.7 \\
\hline $\mathrm{O}(2 \mathrm{~S})-\mathrm{C}(5 \mathrm{~S})-\mathrm{H}(5 \mathrm{SB})$ & 110.1 & $\mathrm{C}(7 \mathrm{~S})-\mathrm{C}(8 \mathrm{~S})-\mathrm{H}(8 \mathrm{~S} 2)$ & 110.7 \\
\hline $\mathrm{C}(6 \mathrm{~S})-\mathrm{C}(5 \mathrm{~S})-\mathrm{H}(5 \mathrm{SB})$ & 110.1 & $\mathrm{H}(8 \mathrm{~S} 1)-\mathrm{C}(8 \mathrm{~S})-\mathrm{H}(8 \mathrm{~S} 2)$ & 108.8 \\
\hline
\end{tabular}




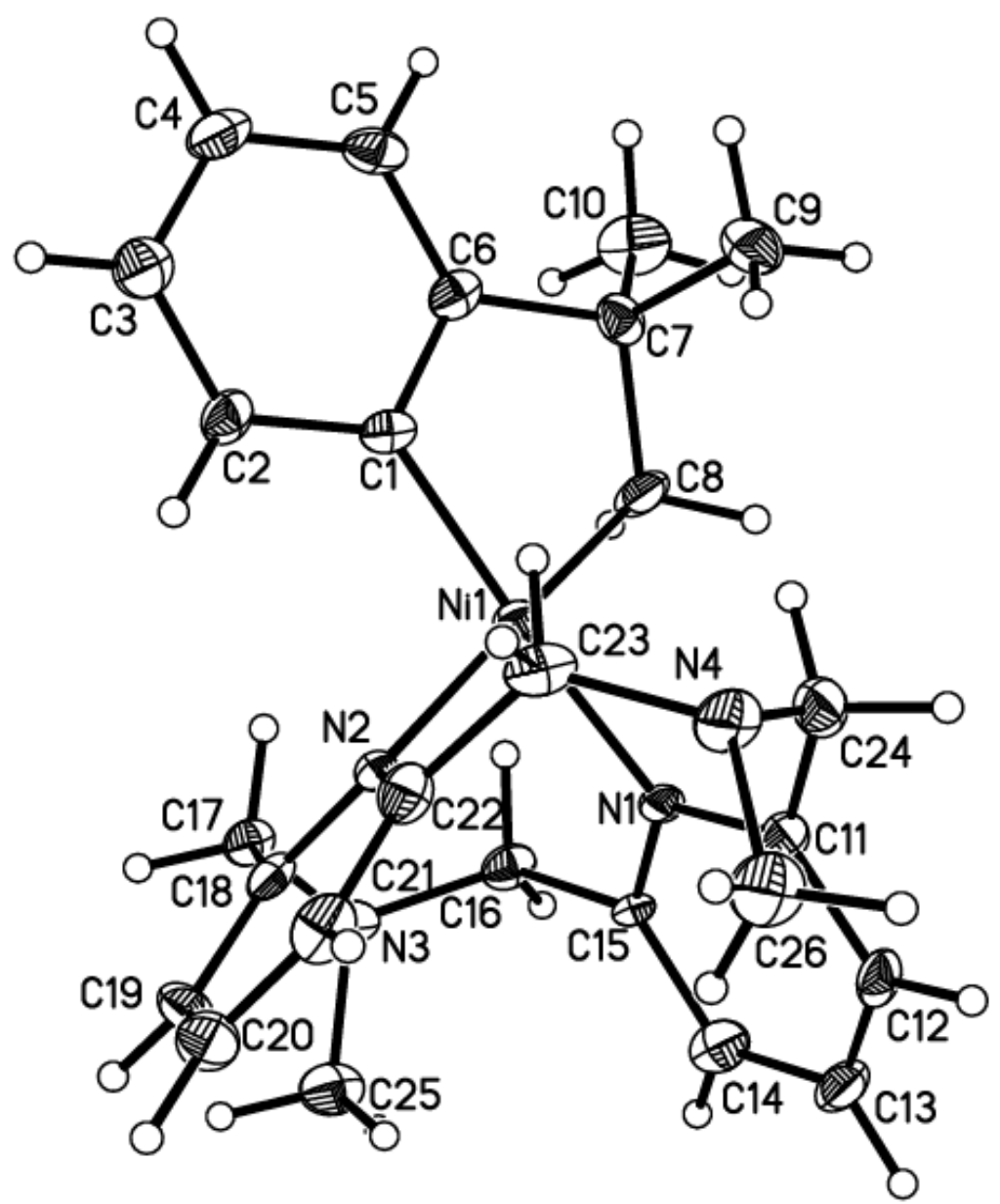

Figure S29. Projection view of 119415 t5 with 50\% thermal ellipsoids. 


\section{$\mathrm{X}$-ray structure determination of [( $\left.{ }^{\mathrm{Me}} \mathrm{N} 4\right) \mathrm{Ni}^{\mathrm{II}}$ (cycloneophyl)]BPh${ }_{4}$}

Table S25. Crystal data and structure refinement for $\operatorname{lm} 9315$.

Identification code

Empirical formula

Formula weight

Temperature

Wavelength

Crystal system

Space group

Unit cell dimensions

Volume

Z

Density (calculated)

Absorption coefficient

$\mathrm{F}(000)$

Crystal size

Theta range for data collection

Index ranges

Reflections collected

Independent reflections

Completeness to theta $=25.242^{\circ}$

Absorption correction

Max. and min. transmission

Refinement method

Data / restraints / parameters

Goodness-of-fit on $\mathrm{F}^{2}$

Final $\mathrm{R}$ indices $[\mathrm{I}>2 \operatorname{sigma}(\mathrm{I})]$

$\mathrm{R}$ indices (all data)

Extinction coefficient

Largest diff. peak and hole
19315/lt/smart/JS-050415-01

$\mathrm{C}_{62} \mathrm{H}_{76} \mathrm{~B} \mathrm{~N}_{4} \mathrm{Ni} \mathrm{O}_{3}$

994.78

100(2) K

$0.71073 \AA$

Monoclinic

$\mathrm{P} 21 / \mathrm{c}$

$\mathrm{a}=18.0244(6) \AA$ $\alpha=90^{\circ}$.

$\mathrm{b}=12.6029(4) \AA$ $\beta=98.4100(16)^{\circ}$.

$\mathrm{c}=23.9084(8) \AA$ $\gamma=90^{\circ}$.
4

$1.230 \mathrm{Mg} / \mathrm{m}^{3}$

$0.410 \mathrm{~mm}^{-1}$

2132

$0.572 \times 0.194 \times 0.072 \mathrm{~mm}^{3}$

1.722 to $27.512^{\circ}$.

$-23 \leq \mathrm{h} \leq 23,-8 \leq \mathrm{k} \leq 16,-31 \leq \mathrm{l} \leq 30$

84276

$12338[\mathrm{R}(\mathrm{int})=0.0678]$

$100.0 \%$

Semi-empirical from equivalents

0.8621 and 0.7578

Full-matrix least-squares on $\mathrm{F}^{2}$

12338 / 114 / 645

1.031

$\mathrm{R} 1=0.0613, \mathrm{wR} 2=0.1446$

$\mathrm{R} 1=0.0970, \mathrm{wR} 2=0.1660$

$\mathrm{n} / \mathrm{a}$

0.858 and -0.570 e. $\AA^{-3}$ 
Table S26. Bond lengths $[\AA]$ and angles $\left[{ }^{\circ}\right]$ for $\operatorname{lm} 9315$.

\begin{tabular}{|c|c|c|c|}
\hline Ni(1)-C(18) & $1.920(3)$ & $C(11)-C(12)$ & $1.398(4)$ \\
\hline $\mathrm{Ni}(1)-\mathrm{C}(17)$ & $1.973(3)$ & $\mathrm{C}(11)-\mathrm{H}(11)$ & 0.9500 \\
\hline Ni(1)-N(2) & $1.997(2)$ & $C(12)-C(13)$ & $1.489(5)$ \\
\hline $\mathrm{Ni}(1)-\mathrm{N}(1)$ & $2.001(2)$ & $\mathrm{C}(13)-\mathrm{H}(13 \mathrm{~A})$ & 0.9900 \\
\hline $\mathrm{Ni}(1)-\mathrm{N}(3)$ & $2.241(2)$ & $\mathrm{C}(13)-\mathrm{H}(13 \mathrm{~B})$ & 0.9900 \\
\hline Ni(1)-N(4) & $2.266(3)$ & $\mathrm{C}(14)-\mathrm{H}(14 \mathrm{~A})$ & 0.9900 \\
\hline $\mathrm{N}(1)-\mathrm{C}(5)$ & $1.340(3)$ & $\mathrm{C}(14)-\mathrm{H}(14 \mathrm{~B})$ & 0.9900 \\
\hline $\mathrm{N}(1)-\mathrm{C}(1)$ & $1.345(4)$ & $\mathrm{C}(15)-\mathrm{H}(15 \mathrm{~A})$ & 0.9800 \\
\hline $\mathrm{N}(2)-\mathrm{C}(8)$ & $1.338(4)$ & $\mathrm{C}(15)-\mathrm{H}(15 \mathrm{~B})$ & 0.9800 \\
\hline $\mathrm{N}(2)-\mathrm{C}(12)$ & $1.346(4)$ & $\mathrm{C}(15)-\mathrm{H}(15 \mathrm{C})$ & 0.9800 \\
\hline $\mathrm{N}(3)-\mathrm{C}(15)$ & $1.478(3)$ & $\mathrm{C}(16)-\mathrm{H}(16 \mathrm{~A})$ & 0.9800 \\
\hline $\mathrm{N}(3)-\mathrm{C}(7)$ & $1.484(4)$ & $\mathrm{C}(16)-\mathrm{H}(16 \mathrm{~B})$ & 0.9800 \\
\hline $\mathrm{N}(3)-\mathrm{C}(6)$ & $1.485(4)$ & $\mathrm{C}(16)-\mathrm{H}(16 \mathrm{C})$ & 0.9800 \\
\hline $\mathrm{N}(4)-\mathrm{C}(16)$ & $1.474(4)$ & $\mathrm{C}(17)-\mathrm{C}(24)$ & $1.551(4)$ \\
\hline $\mathrm{N}(4)-\mathrm{C}(13)$ & $1.488(4)$ & $\mathrm{C}(17)-\mathrm{H}(17 \mathrm{~A})$ & 0.9900 \\
\hline $\mathrm{N}(4)-\mathrm{C}(14)$ & $1.490(4)$ & $\mathrm{C}(17)-\mathrm{H}(17 \mathrm{~B})$ & 0.9900 \\
\hline$C(1)-C(2)$ & $1.386(4)$ & $\mathrm{C}(18)-\mathrm{C}(19)$ & $1.393(4)$ \\
\hline$C(1)-C(14)$ & $1.507(4)$ & $\mathrm{C}(18)-\mathrm{C}(23)$ & $1.403(4)$ \\
\hline$C(2)-C(3)$ & $1.380(4)$ & $C(19)-C(20)$ & $1.393(5)$ \\
\hline $\mathrm{C}(2)-\mathrm{H}(2)$ & 0.9500 & $\mathrm{C}(19)-\mathrm{H}(19)$ & 0.9500 \\
\hline$C(3)-C(4)$ & $1.388(4)$ & $C(20)-C(21)$ & $1.381(5)$ \\
\hline $\mathrm{C}(3)-\mathrm{H}(3)$ & 0.9500 & $\mathrm{C}(20)-\mathrm{H}(20)$ & 0.9500 \\
\hline$C(4)-C(5)$ & $1.383(4)$ & $C(21)-C(22)$ & $1.384(5)$ \\
\hline $\mathrm{C}(4)-\mathrm{H}(4)$ & 0.9500 & $\mathrm{C}(21)-\mathrm{H}(21)$ & 0.9500 \\
\hline$C(5)-C(6)$ & $1.502(4)$ & $C(22)-C(23)$ & $1.401(4)$ \\
\hline $\mathrm{C}(6)-\mathrm{H}(6 \mathrm{~A})$ & 0.9900 & $\mathrm{C}(22)-\mathrm{H}(22)$ & 0.9500 \\
\hline $\mathrm{C}(6)-\mathrm{H}(6 \mathrm{~B})$ & 0.9900 & $C(23)-C(24)$ & $1.509(4)$ \\
\hline$C(7)-C(8)$ & $1.494(4)$ & $C(24)-C(25)$ & $1.539(4)$ \\
\hline $\mathrm{C}(7)-\mathrm{H}(7 \mathrm{~A})$ & 0.9900 & $C(24)-C(26)$ & $1.539(4)$ \\
\hline $\mathrm{C}(7)-\mathrm{H}(7 \mathrm{~B})$ & 0.9900 & $\mathrm{C}(25)-\mathrm{H}(25 \mathrm{~A})$ & 0.9800 \\
\hline $\mathrm{C}(8)-\mathrm{C}(9)$ & $1.395(4)$ & $\mathrm{C}(25)-\mathrm{H}(25 \mathrm{~B})$ & 0.9800 \\
\hline$C(9)-C(10)$ & $1.369(5)$ & $\mathrm{C}(25)-\mathrm{H}(25 \mathrm{C})$ & 0.9800 \\
\hline $\mathrm{C}(9)-\mathrm{H}(9)$ & 0.9500 & $\mathrm{C}(26)-\mathrm{H}(26 \mathrm{~A})$ & 0.9800 \\
\hline$C(10)-C(11)$ & $1.374(5)$ & $\mathrm{C}(26)-\mathrm{H}(26 \mathrm{~B})$ & 0.9800 \\
\hline $\mathrm{C}(10)-\mathrm{H}(10)$ & 0.9500 & $\mathrm{C}(26)-\mathrm{H}(26 \mathrm{C})$ & 0.9800 \\
\hline
\end{tabular}




\begin{tabular}{|c|c|c|c|}
\hline$C(27)-C(32)$ & $1.405(4)$ & $C(45)-C(50)$ & $1.399(4)$ \\
\hline $\mathrm{C}(27)-\mathrm{C}(28)$ & $1.408(4)$ & $C(45)-C(46)$ & $1.406(4)$ \\
\hline $\mathrm{C}(27)-\mathrm{B}(1)$ & $1.648(4)$ & $\mathrm{C}(45)-\mathrm{B}(1)$ & $1.655(4)$ \\
\hline $\mathrm{C}(28)-\mathrm{C}(29)$ & $1.388(4)$ & $C(46)-C(47)$ & $1.391(4)$ \\
\hline $\mathrm{C}(28)-\mathrm{H}(28)$ & 0.9500 & $\mathrm{C}(46)-\mathrm{H}(46)$ & 0.9500 \\
\hline$C(29)-C(30)$ & $1.386(4)$ & $C(47)-C(48)$ & $1.382(5)$ \\
\hline $\mathrm{C}(29)-\mathrm{H}(29)$ & 0.9500 & $\mathrm{C}(47)-\mathrm{H}(47)$ & 0.9500 \\
\hline$C(30)-C(31)$ & $1.383(4)$ & $\mathrm{C}(48)-\mathrm{C}(49)$ & $1.391(5)$ \\
\hline $\mathrm{C}(30)-\mathrm{H}(30)$ & 0.9500 & $\mathrm{C}(48)-\mathrm{H}(48)$ & 0.9500 \\
\hline$C(31)-C(32)$ & $1.394(4)$ & $C(49)-C(50)$ & $1.395(4)$ \\
\hline $\mathrm{C}(31)-\mathrm{H}(31)$ & 0.9500 & $\mathrm{C}(49)-\mathrm{H}(49)$ & 0.9500 \\
\hline $\mathrm{C}(32)-\mathrm{H}(32)$ & 0.9500 & $\mathrm{C}(50)-\mathrm{H}(50)$ & 0.9500 \\
\hline$C(33)-C(38)$ & $1.401(4)$ & $\mathrm{O}(1 \mathrm{~S})-\mathrm{C}(1 \mathrm{~S})$ & $1.388(7)$ \\
\hline$C(33)-C(34)$ & $1.412(4)$ & $\mathrm{O}(1 \mathrm{~S})-\mathrm{C}(4 \mathrm{~S})$ & $1.495(8)$ \\
\hline $\mathrm{C}(33)-\mathrm{B}(1)$ & $1.654(4)$ & $\mathrm{C}(1 \mathrm{~S})-\mathrm{O}\left(1 \mathrm{~S}^{\prime}\right)$ & $1.429(16)$ \\
\hline$C(34)-C(35)$ & $1.396(4)$ & $C(1 S)-C(2 S)$ & $1.472(7)$ \\
\hline $\mathrm{C}(34)-\mathrm{H}(34)$ & 0.9500 & $\mathrm{C}(1 \mathrm{~S})-\mathrm{H}(1 \mathrm{SA})$ & 0.9900 \\
\hline$C(35)-C(36)$ & $1.383(5)$ & $\mathrm{C}(1 \mathrm{~S})-\mathrm{H}(1 \mathrm{SB})$ & 0.9900 \\
\hline $\mathrm{C}(35)-\mathrm{H}(35)$ & 0.9500 & $\mathrm{C}(2 \mathrm{~S})-\mathrm{C}(3 \mathrm{~S})$ & $1.510(7)$ \\
\hline$C(36)-C(37)$ & $1.381(5)$ & $\mathrm{C}(2 \mathrm{~S})-\mathrm{H}(2 \mathrm{SA})$ & 0.9900 \\
\hline $\mathrm{C}(36)-\mathrm{H}(36)$ & 0.9500 & $\mathrm{C}(2 \mathrm{~S})-\mathrm{H}(2 \mathrm{SB})$ & 0.9900 \\
\hline $\mathrm{C}(37)-\mathrm{C}(38)$ & $1.386(4)$ & $C(3 S)-C(4 S)$ & $1.414(7)$ \\
\hline $\mathrm{C}(37)-\mathrm{H}(37)$ & 0.9500 & $\mathrm{C}(3 \mathrm{~S})-\mathrm{H}(3 \mathrm{SA})$ & 0.9900 \\
\hline $\mathrm{C}(38)-\mathrm{H}(38)$ & 0.9500 & $\mathrm{C}(3 \mathrm{~S})-\mathrm{H}(3 \mathrm{SB})$ & 0.9900 \\
\hline$C(39)-C(44)$ & $1.400(4)$ & $\mathrm{C}(4 \mathrm{~S})-\mathrm{O}\left(1 \mathrm{~S}^{\prime}\right)$ & $1.233(15)$ \\
\hline$C(39)-C(40)$ & $1.406(4)$ & $\mathrm{C}(4 \mathrm{~S})-\mathrm{H}(4 \mathrm{SA})$ & 0.9900 \\
\hline $\mathrm{C}(39)-\mathrm{B}(1)$ & $1.638(4)$ & $\mathrm{C}(4 \mathrm{~S})-\mathrm{H}(4 \mathrm{SB})$ & 0.9900 \\
\hline$C(40)-C(41)$ & $1.392(4)$ & $\mathrm{O}(2 \mathrm{~S})-\mathrm{C}(8 \mathrm{~S})$ & $1.378(7)$ \\
\hline $\mathrm{C}(40)-\mathrm{H}(40)$ & 0.9500 & $\mathrm{O}(2 \mathrm{~S})-\mathrm{C}(5 \mathrm{~S})$ & $1.468(8)$ \\
\hline$C(41)-C(42)$ & $1.382(5)$ & $C(5 S)-C(6 S)$ & $1.416(10)$ \\
\hline $\mathrm{C}(41)-\mathrm{H}(41)$ & 0.9500 & $\mathrm{C}(5 \mathrm{~S})-\mathrm{O}\left(2 \mathrm{~S}^{\prime}\right)$ & $1.501(15)$ \\
\hline$C(42)-C(43)$ & $1.381(5)$ & $\mathrm{C}(5 \mathrm{~S})-\mathrm{H}(5 \mathrm{~S} 1)$ & 0.9900 \\
\hline $\mathrm{C}(42)-\mathrm{H}(42)$ & 0.9500 & $\mathrm{C}(5 \mathrm{~S})-\mathrm{H}(5 \mathrm{~S} 2)$ & 0.9900 \\
\hline$C(43)-C(44)$ & $1.396(4)$ & $C(6 S)-C(7 S)$ & $1.468(9)$ \\
\hline $\mathrm{C}(43)-\mathrm{H}(43)$ & 0.9500 & $\mathrm{C}(6 \mathrm{~S})-\mathrm{H}(6 \mathrm{SA})$ & 0.9900 \\
\hline $\mathrm{C}(44)-\mathrm{H}(44)$ & 0.9500 & $\mathrm{C}(6 \mathrm{~S})-\mathrm{H}(6 \mathrm{SB})$ & 0.9900 \\
\hline
\end{tabular}




\begin{tabular}{|c|c|c|c|}
\hline $\mathrm{C}(7 \mathrm{~S})-\mathrm{C}(8 \mathrm{~S})$ & $1.502(8)$ & $\mathrm{C}(1)-\mathrm{N}(1)-\mathrm{Ni}(1)$ & 119.31(19) \\
\hline C(7S)-H(7SA) & 0.9900 & $\mathrm{C}(8)-\mathrm{N}(2)-\mathrm{C}(12)$ & $119.9(3)$ \\
\hline $\mathrm{C}(7 \mathrm{~S})-\mathrm{H}(7 \mathrm{SB})$ & 0.9900 & $\mathrm{C}(8)-\mathrm{N}(2)-\mathrm{Ni}(1)$ & $119.0(2)$ \\
\hline $\mathrm{C}(8 \mathrm{~S})-\mathrm{O}\left(2 \mathrm{~S}^{\prime}\right)$ & $1.666(16)$ & $\mathrm{C}(12)-\mathrm{N}(2)-\mathrm{Ni}(1)$ & $118.8(2)$ \\
\hline $\mathrm{C}(8 \mathrm{~S})-\mathrm{H}(8 \mathrm{~S} 1)$ & 0.9900 & $\mathrm{C}(15)-\mathrm{N}(3)-\mathrm{C}(7)$ & $109.8(2)$ \\
\hline $\mathrm{C}(8 \mathrm{~S})-\mathrm{H}(8 \mathrm{~S} 2)$ & 0.9900 & $\mathrm{C}(15)-\mathrm{N}(3)-\mathrm{C}(6)$ & $108.6(2)$ \\
\hline $\mathrm{O}(3 \mathrm{~S})-\mathrm{C}(12 \mathrm{~S})$ & $1.395(6)$ & $C(7)-N(3)-C(6)$ & $111.3(2)$ \\
\hline $\mathrm{O}(3 \mathrm{~S})-\mathrm{C}(9 \mathrm{~S})$ & $1.399(6)$ & $\mathrm{C}(15)-\mathrm{N}(3)-\mathrm{Ni}(1)$ & $116.63(17)$ \\
\hline $\mathrm{C}(9 \mathrm{~S})-\mathrm{C}(10 \mathrm{~S})$ & $1.490(6)$ & $\mathrm{C}(7)-\mathrm{N}(3)-\mathrm{Ni}(1)$ & $107.22(17)$ \\
\hline $\mathrm{C}(9 \mathrm{~S})-\mathrm{H}(9 \mathrm{SA})$ & 0.9900 & $\mathrm{C}(6)-\mathrm{N}(3)-\mathrm{Ni}(1)$ & $103.21(16)$ \\
\hline $\mathrm{C}(9 \mathrm{~S})-\mathrm{H}(9 \mathrm{SB})$ & 0.9900 & $\mathrm{C}(16)-\mathrm{N}(4)-\mathrm{C}(13)$ & 109.7(3) \\
\hline$C(10 S)-C(11 S)$ & $1.504(6)$ & $\mathrm{C}(16)-\mathrm{N}(4)-\mathrm{C}(14)$ & $108.9(3)$ \\
\hline $\mathrm{C}(10 \mathrm{~S})-\mathrm{H}(10 \mathrm{~A})$ & 0.9900 & $\mathrm{C}(13)-\mathrm{N}(4)-\mathrm{C}(14)$ & $112.2(3)$ \\
\hline $\mathrm{C}(10 \mathrm{~S})-\mathrm{H}(10 \mathrm{~B})$ & 0.9900 & $\mathrm{C}(16)-\mathrm{N}(4)-\mathrm{Ni}(1)$ & $116.6(2)$ \\
\hline$C(11 S)-C(12 S)$ & $1.489(7)$ & $\mathrm{C}(13)-\mathrm{N}(4)-\mathrm{Ni}(1)$ & $103.31(18)$ \\
\hline $\mathrm{C}(11 \mathrm{~S})-\mathrm{H}(11 \mathrm{~A})$ & 0.9900 & $\mathrm{C}(14)-\mathrm{N}(4)-\mathrm{Ni}(1)$ & $106.08(18)$ \\
\hline $\mathrm{C}(11 \mathrm{~S})-\mathrm{H}(11 \mathrm{~B})$ & 0.9900 & $\mathrm{~N}(1)-\mathrm{C}(1)-\mathrm{C}(2)$ & $121.0(3)$ \\
\hline $\mathrm{C}(12 \mathrm{~S})-\mathrm{H}(12 \mathrm{~A})$ & 0.9900 & $\mathrm{~N}(1)-\mathrm{C}(1)-\mathrm{C}(14)$ & $115.2(3)$ \\
\hline $\mathrm{C}(12 \mathrm{~S})-\mathrm{H}(12 \mathrm{~B})$ & 0.9900 & $C(2)-C(1)-C(14)$ & $123.7(3)$ \\
\hline $\mathrm{C}(18)-\mathrm{Ni}(1)-\mathrm{C}(17)$ & $82.88(12)$ & $\mathrm{C}(3)-\mathrm{C}(2)-\mathrm{C}(1)$ & $119.0(3)$ \\
\hline $\mathrm{C}(18)-\mathrm{Ni}(1)-\mathrm{N}(2)$ & $177.67(11)$ & $\mathrm{C}(3)-\mathrm{C}(2)-\mathrm{H}(2)$ & 120.5 \\
\hline $\mathrm{C}(17)-\mathrm{Ni}(1)-\mathrm{N}(2)$ & $95.35(10)$ & $\mathrm{C}(1)-\mathrm{C}(2)-\mathrm{H}(2)$ & 120.5 \\
\hline $\mathrm{C}(18)-\mathrm{Ni}(1)-\mathrm{N}(1)$ & $99.00(11)$ & $C(2)-C(3)-C(4)$ & $119.8(3)$ \\
\hline $\mathrm{C}(17)-\mathrm{Ni}(1)-\mathrm{N}(1)$ & $178.08(11)$ & $\mathrm{C}(2)-\mathrm{C}(3)-\mathrm{H}(3)$ & 120.1 \\
\hline $\mathrm{N}(2)-\mathrm{Ni}(1)-\mathrm{N}(1)$ & $82.78(9)$ & $\mathrm{C}(4)-\mathrm{C}(3)-\mathrm{H}(3)$ & 120.1 \\
\hline $\mathrm{C}(18)-\mathrm{Ni}(1)-\mathrm{N}(3)$ & $102.09(11)$ & $C(5)-C(4)-C(3)$ & $118.4(3)$ \\
\hline $\mathrm{C}(17)-\mathrm{Ni}(1)-\mathrm{N}(3)$ & $99.39(10)$ & $\mathrm{C}(5)-\mathrm{C}(4)-\mathrm{H}(4)$ & 120.8 \\
\hline $\mathrm{N}(2)-\mathrm{Ni}(1)-\mathrm{N}(3)$ & $79.68(9)$ & $\mathrm{C}(3)-\mathrm{C}(4)-\mathrm{H}(4)$ & 120.8 \\
\hline N(1)-Ni(1)-N(3) & $79.84(9)$ & $\mathrm{N}(1)-\mathrm{C}(5)-\mathrm{C}(4)$ & $121.8(3)$ \\
\hline $\mathrm{C}(18)-\mathrm{Ni}(1)-\mathrm{N}(4)$ & $99.36(11)$ & $\mathrm{N}(1)-\mathrm{C}(5)-\mathrm{C}(6)$ & $114.3(2)$ \\
\hline $\mathrm{C}(17)-\mathrm{Ni}(1)-\mathrm{N}(4)$ & $100.34(11)$ & $C(4)-C(5)-C(6)$ & $124.0(3)$ \\
\hline $\mathrm{N}(2)-\mathrm{Ni}(1)-\mathrm{N}(4)$ & 79.42(10) & $\mathrm{N}(3)-\mathrm{C}(6)-\mathrm{C}(5)$ & $111.1(2)$ \\
\hline $\mathrm{N}(1)-\mathrm{Ni}(1)-\mathrm{N}(4)$ & $79.82(9)$ & $\mathrm{N}(3)-\mathrm{C}(6)-\mathrm{H}(6 \mathrm{~A})$ & 109.4 \\
\hline $\mathrm{N}(3)-\mathrm{Ni}(1)-\mathrm{N}(4)$ & $152.39(9)$ & $C(5)-C(6)-H(6 A)$ & 109.4 \\
\hline $\mathrm{C}(5)-\mathrm{N}(1)-\mathrm{C}(1)$ & $120.0(2)$ & $\mathrm{N}(3)-\mathrm{C}(6)-\mathrm{H}(6 \mathrm{~B})$ & 109.4 \\
\hline $\mathrm{C}(5)-\mathrm{N}(1)-\mathrm{Ni}(1)$ & $118.05(18)$ & $\mathrm{C}(5)-\mathrm{C}(6)-\mathrm{H}(6 \mathrm{~B})$ & 109.4 \\
\hline
\end{tabular}




\begin{tabular}{|c|c|c|c|}
\hline $\mathrm{H}(6 \mathrm{~A})-\mathrm{C}(6)-\mathrm{H}(6 \mathrm{~B})$ & 108.0 & $\mathrm{H}(15 \mathrm{~A})-\mathrm{C}(15)-\mathrm{H}(15 \mathrm{~B})$ & 109.5 \\
\hline $\mathrm{N}(3)-\mathrm{C}(7)-\mathrm{C}(8)$ & $112.5(2)$ & $\mathrm{N}(3)-\mathrm{C}(15)-\mathrm{H}(15 \mathrm{C})$ & 109.5 \\
\hline $\mathrm{N}(3)-\mathrm{C}(7)-\mathrm{H}(7 \mathrm{~A})$ & 109.1 & $\mathrm{H}(15 \mathrm{~A})-\mathrm{C}(15)-\mathrm{H}(15 \mathrm{C})$ & 109.5 \\
\hline $\mathrm{C}(8)-\mathrm{C}(7)-\mathrm{H}(7 \mathrm{~A})$ & 109.1 & $\mathrm{H}(15 \mathrm{~B})-\mathrm{C}(15)-\mathrm{H}(15 \mathrm{C})$ & 109.5 \\
\hline $\mathrm{N}(3)-\mathrm{C}(7)-\mathrm{H}(7 \mathrm{~B})$ & 109.1 & $\mathrm{~N}(4)-\mathrm{C}(16)-\mathrm{H}(16 \mathrm{~A})$ & 109.5 \\
\hline $\mathrm{C}(8)-\mathrm{C}(7)-\mathrm{H}(7 \mathrm{~B})$ & 109.1 & $\mathrm{~N}(4)-\mathrm{C}(16)-\mathrm{H}(16 \mathrm{~B})$ & 109.5 \\
\hline $\mathrm{H}(7 \mathrm{~A})-\mathrm{C}(7)-\mathrm{H}(7 \mathrm{~B})$ & 107.8 & $\mathrm{H}(16 \mathrm{~A})-\mathrm{C}(16)-\mathrm{H}(16 \mathrm{~B})$ & 109.5 \\
\hline $\mathrm{N}(2)-\mathrm{C}(8)-\mathrm{C}(9)$ & $121.6(3)$ & $\mathrm{N}(4)-\mathrm{C}(16)-\mathrm{H}(16 \mathrm{C})$ & 109.5 \\
\hline $\mathrm{N}(2)-\mathrm{C}(8)-\mathrm{C}(7)$ & $116.0(3)$ & $\mathrm{H}(16 \mathrm{~A})-\mathrm{C}(16)-\mathrm{H}(16 \mathrm{C})$ & 109.5 \\
\hline C(9)-C(8)-C(7) & $122.3(3)$ & $\mathrm{H}(16 \mathrm{~B})-\mathrm{C}(16)-\mathrm{H}(16 \mathrm{C})$ & 109.5 \\
\hline$C(10)-C(9)-C(8)$ & $118.6(3)$ & $\mathrm{C}(24)-\mathrm{C}(17)-\mathrm{Ni}(1)$ & $116.21(19)$ \\
\hline $\mathrm{C}(10)-\mathrm{C}(9)-\mathrm{H}(9)$ & 120.7 & $\mathrm{C}(24)-\mathrm{C}(17)-\mathrm{H}(17 \mathrm{~A})$ & 108.2 \\
\hline $\mathrm{C}(8)-\mathrm{C}(9)-\mathrm{H}(9)$ & 120.7 & Ni(1)-C(17)-H(17A) & 108.2 \\
\hline$C(9)-C(10)-C(11)$ & $120.1(3)$ & $\mathrm{C}(24)-\mathrm{C}(17)-\mathrm{H}(17 \mathrm{~B})$ & 108.2 \\
\hline $\mathrm{C}(9)-\mathrm{C}(10)-\mathrm{H}(10)$ & 119.9 & Ni(1)-C(17)-H(17B) & 108.2 \\
\hline $\mathrm{C}(11)-\mathrm{C}(10)-\mathrm{H}(10)$ & 119.9 & $\mathrm{H}(17 \mathrm{~A})-\mathrm{C}(17)-\mathrm{H}(17 \mathrm{~B})$ & 107.4 \\
\hline$C(10)-C(11)-C(12)$ & $119.0(3)$ & C(19)-C(18)-C(23) & $118.5(3)$ \\
\hline $\mathrm{C}(10)-\mathrm{C}(11)-\mathrm{H}(11)$ & 120.5 & $\mathrm{C}(19)-\mathrm{C}(18)-\mathrm{Ni}(1)$ & $123.2(2)$ \\
\hline $\mathrm{C}(12)-\mathrm{C}(11)-\mathrm{H}(11)$ & 120.5 & $\mathrm{C}(23)-\mathrm{C}(18)-\mathrm{Ni}(1)$ & $118.2(2)$ \\
\hline $\mathrm{N}(2)-\mathrm{C}(12)-\mathrm{C}(11)$ & $120.7(3)$ & $C(20)-C(19)-C(18)$ & $121.3(3)$ \\
\hline $\mathrm{N}(2)-\mathrm{C}(12)-\mathrm{C}(13)$ & $114.8(3)$ & $\mathrm{C}(20)-\mathrm{C}(19)-\mathrm{H}(19)$ & 119.4 \\
\hline $\mathrm{C}(11)-\mathrm{C}(12)-\mathrm{C}(13)$ & $124.5(3)$ & $\mathrm{C}(18)-\mathrm{C}(19)-\mathrm{H}(19)$ & 119.4 \\
\hline $\mathrm{N}(4)-\mathrm{C}(13)-\mathrm{C}(12)$ & $112.0(3)$ & $\mathrm{C}(21)-\mathrm{C}(20)-\mathrm{C}(19)$ & $119.8(3)$ \\
\hline $\mathrm{N}(4)-\mathrm{C}(13)-\mathrm{H}(13 \mathrm{~A})$ & 109.2 & $\mathrm{C}(21)-\mathrm{C}(20)-\mathrm{H}(20)$ & 120.1 \\
\hline $\mathrm{C}(12)-\mathrm{C}(13)-\mathrm{H}(13 \mathrm{~A})$ & 109.2 & $\mathrm{C}(19)-\mathrm{C}(20)-\mathrm{H}(20)$ & 120.1 \\
\hline $\mathrm{N}(4)-\mathrm{C}(13)-\mathrm{H}(13 \mathrm{~B})$ & 109.2 & $C(20)-C(21)-C(22)$ & $120.0(3)$ \\
\hline $\mathrm{C}(12)-\mathrm{C}(13)-\mathrm{H}(13 \mathrm{~B})$ & 109.2 & $\mathrm{C}(20)-\mathrm{C}(21)-\mathrm{H}(21)$ & 120.0 \\
\hline $\mathrm{H}(13 \mathrm{~A})-\mathrm{C}(13)-\mathrm{H}(13 \mathrm{~B})$ & 107.9 & $\mathrm{C}(22)-\mathrm{C}(21)-\mathrm{H}(21)$ & 120.0 \\
\hline $\mathrm{N}(4)-\mathrm{C}(14)-\mathrm{C}(1)$ & $112.9(2)$ & $\mathrm{C}(21)-\mathrm{C}(22)-\mathrm{C}(23)$ & $120.5(3)$ \\
\hline $\mathrm{N}(4)-\mathrm{C}(14)-\mathrm{H}(14 \mathrm{~A})$ & 109.0 & $\mathrm{C}(21)-\mathrm{C}(22)-\mathrm{H}(22)$ & 119.7 \\
\hline $\mathrm{C}(1)-\mathrm{C}(14)-\mathrm{H}(14 \mathrm{~A})$ & 109.0 & $\mathrm{C}(23)-\mathrm{C}(22)-\mathrm{H}(22)$ & 119.7 \\
\hline $\mathrm{N}(4)-\mathrm{C}(14)-\mathrm{H}(14 \mathrm{~B})$ & 109.0 & $C(22)-C(23)-C(18)$ & $119.8(3)$ \\
\hline $\mathrm{C}(1)-\mathrm{C}(14)-\mathrm{H}(14 \mathrm{~B})$ & 109.0 & $C(22)-C(23)-C(24)$ & $123.7(3)$ \\
\hline $\mathrm{H}(14 \mathrm{~A})-\mathrm{C}(14)-\mathrm{H}(14 \mathrm{~B})$ & 107.8 & $C(18)-C(23)-C(24)$ & $116.4(3)$ \\
\hline $\mathrm{N}(3)-\mathrm{C}(15)-\mathrm{H}(15 \mathrm{~A})$ & 109.5 & $C(23)-C(24)-C(25)$ & $110.3(2)$ \\
\hline $\mathrm{N}(3)-\mathrm{C}(15)-\mathrm{H}(15 \mathrm{~B})$ & 109.5 & $C(23)-C(24)-C(26)$ & $111.1(3)$ \\
\hline
\end{tabular}




\begin{tabular}{|c|c|c|c|}
\hline$C(25)-C(24)-C(26)$ & $107.9(2)$ & $\mathrm{C}(34)-\mathrm{C}(33)-\mathrm{B}(1)$ & $121.3(3)$ \\
\hline $\mathrm{C}(23)-\mathrm{C}(24)-\mathrm{C}(17)$ & $106.2(2)$ & $\mathrm{C}(35)-\mathrm{C}(34)-\mathrm{C}(33)$ & $122.7(3)$ \\
\hline$C(25)-C(24)-C(17)$ & $110.2(2)$ & $\mathrm{C}(35)-\mathrm{C}(34)-\mathrm{H}(34)$ & 118.6 \\
\hline$C(26)-C(24)-C(17)$ & 111.2(2) & $\mathrm{C}(33)-\mathrm{C}(34)-\mathrm{H}(34)$ & 118.6 \\
\hline $\mathrm{C}(24)-\mathrm{C}(25)-\mathrm{H}(25 \mathrm{~A})$ & 109.5 & $C(36)-C(35)-C(34)$ & $120.1(3)$ \\
\hline $\mathrm{C}(24)-\mathrm{C}(25)-\mathrm{H}(25 \mathrm{~B})$ & 109.5 & $\mathrm{C}(36)-\mathrm{C}(35)-\mathrm{H}(35)$ & 120.0 \\
\hline $\mathrm{H}(25 \mathrm{~A})-\mathrm{C}(25)-\mathrm{H}(25 \mathrm{~B})$ & 109.5 & $\mathrm{C}(34)-\mathrm{C}(35)-\mathrm{H}(35)$ & 120.0 \\
\hline $\mathrm{C}(24)-\mathrm{C}(25)-\mathrm{H}(25 \mathrm{C})$ & 109.5 & $\mathrm{C}(37)-\mathrm{C}(36)-\mathrm{C}(35)$ & $118.9(3)$ \\
\hline $\mathrm{H}(25 \mathrm{~A})-\mathrm{C}(25)-\mathrm{H}(25 \mathrm{C})$ & 109.5 & $\mathrm{C}(37)-\mathrm{C}(36)-\mathrm{H}(36)$ & 120.5 \\
\hline $\mathrm{H}(25 \mathrm{~B})-\mathrm{C}(25)-\mathrm{H}(25 \mathrm{C})$ & 109.5 & $\mathrm{C}(35)-\mathrm{C}(36)-\mathrm{H}(36)$ & 120.5 \\
\hline$C(24)-C(26)-H(26 A)$ & 109.5 & $\mathrm{C}(36)-\mathrm{C}(37)-\mathrm{C}(38)$ & $120.4(3)$ \\
\hline $\mathrm{C}(24)-\mathrm{C}(26)-\mathrm{H}(26 \mathrm{~B})$ & 109.5 & $\mathrm{C}(36)-\mathrm{C}(37)-\mathrm{H}(37)$ & 119.8 \\
\hline $\mathrm{H}(26 \mathrm{~A})-\mathrm{C}(26)-\mathrm{H}(26 \mathrm{~B})$ & 109.5 & $\mathrm{C}(38)-\mathrm{C}(37)-\mathrm{H}(37)$ & 119.8 \\
\hline $\mathrm{C}(24)-\mathrm{C}(26)-\mathrm{H}(26 \mathrm{C})$ & 109.5 & $\mathrm{C}(37)-\mathrm{C}(38)-\mathrm{C}(33)$ & $123.2(3)$ \\
\hline $\mathrm{H}(26 \mathrm{~A})-\mathrm{C}(26)-\mathrm{H}(26 \mathrm{C})$ & 109.5 & $\mathrm{C}(37)-\mathrm{C}(38)-\mathrm{H}(38)$ & 118.4 \\
\hline $\mathrm{H}(26 \mathrm{~B})-\mathrm{C}(26)-\mathrm{H}(26 \mathrm{C})$ & 109.5 & $\mathrm{C}(33)-\mathrm{C}(38)-\mathrm{H}(38)$ & 118.4 \\
\hline$C(32)-C(27)-C(28)$ & $114.8(3)$ & $C(44)-C(39)-C(40)$ & $115.0(3)$ \\
\hline $\mathrm{C}(32)-\mathrm{C}(27)-\mathrm{B}(1)$ & $124.7(2)$ & $\mathrm{C}(44)-\mathrm{C}(39)-\mathrm{B}(1)$ & $123.6(3)$ \\
\hline $\mathrm{C}(28)-\mathrm{C}(27)-\mathrm{B}(1)$ & $120.3(2)$ & $\mathrm{C}(40)-\mathrm{C}(39)-\mathrm{B}(1)$ & $121.4(3)$ \\
\hline $\mathrm{C}(29)-\mathrm{C}(28)-\mathrm{C}(27)$ & $122.9(3)$ & $C(41)-C(40)-C(39)$ & $122.6(3)$ \\
\hline $\mathrm{C}(29)-\mathrm{C}(28)-\mathrm{H}(28)$ & 118.6 & $\mathrm{C}(41)-\mathrm{C}(40)-\mathrm{H}(40)$ & 118.7 \\
\hline $\mathrm{C}(27)-\mathrm{C}(28)-\mathrm{H}(28)$ & 118.6 & $\mathrm{C}(39)-\mathrm{C}(40)-\mathrm{H}(40)$ & 118.7 \\
\hline$C(30)-C(29)-C(28)$ & $120.2(3)$ & $C(42)-C(41)-C(40)$ & $120.1(3)$ \\
\hline $\mathrm{C}(30)-\mathrm{C}(29)-\mathrm{H}(29)$ & 119.9 & $\mathrm{C}(42)-\mathrm{C}(41)-\mathrm{H}(41)$ & 120.0 \\
\hline $\mathrm{C}(28)-\mathrm{C}(29)-\mathrm{H}(29)$ & 119.9 & $\mathrm{C}(40)-\mathrm{C}(41)-\mathrm{H}(41)$ & 120.0 \\
\hline$C(31)-C(30)-C(29)$ & $118.9(3)$ & $C(43)-C(42)-C(41)$ & $119.5(3)$ \\
\hline $\mathrm{C}(31)-\mathrm{C}(30)-\mathrm{H}(30)$ & 120.5 & $\mathrm{C}(43)-\mathrm{C}(42)-\mathrm{H}(42)$ & 120.2 \\
\hline $\mathrm{C}(29)-\mathrm{C}(30)-\mathrm{H}(30)$ & 120.5 & $\mathrm{C}(41)-\mathrm{C}(42)-\mathrm{H}(42)$ & 120.2 \\
\hline$C(30)-C(31)-C(32)$ & $120.2(3)$ & $C(42)-C(43)-C(44)$ & $119.5(3)$ \\
\hline $\mathrm{C}(30)-\mathrm{C}(31)-\mathrm{H}(31)$ & 119.9 & $\mathrm{C}(42)-\mathrm{C}(43)-\mathrm{H}(43)$ & 120.2 \\
\hline $\mathrm{C}(32)-\mathrm{C}(31)-\mathrm{H}(31)$ & 119.9 & $\mathrm{C}(44)-\mathrm{C}(43)-\mathrm{H}(43)$ & 120.2 \\
\hline $\mathrm{C}(31)-\mathrm{C}(32)-\mathrm{C}(27)$ & $122.8(3)$ & $C(43)-C(44)-C(39)$ & $123.2(3)$ \\
\hline $\mathrm{C}(31)-\mathrm{C}(32)-\mathrm{H}(32)$ & 118.6 & $\mathrm{C}(43)-\mathrm{C}(44)-\mathrm{H}(44)$ & 118.4 \\
\hline $\mathrm{C}(27)-\mathrm{C}(32)-\mathrm{H}(32)$ & 118.6 & $\mathrm{C}(39)-\mathrm{C}(44)-\mathrm{H}(44)$ & 118.4 \\
\hline $\mathrm{C}(38)-\mathrm{C}(33)-\mathrm{C}(34)$ & $114.6(3)$ & $C(50)-C(45)-C(46)$ & $114.8(3)$ \\
\hline $\mathrm{C}(38)-\mathrm{C}(33)-\mathrm{B}(1)$ & $124.1(3)$ & $\mathrm{C}(50)-\mathrm{C}(45)-\mathrm{B}(1)$ & $123.5(2)$ \\
\hline
\end{tabular}




\begin{tabular}{|c|c|c|c|}
\hline$C(46)-C(45)-B(1)$ & $121.5(3)$ & $\mathrm{C}(4 \mathrm{~S})-\mathrm{C}(3 \mathrm{~S})-\mathrm{C}(2 \mathrm{~S})$ & $103.1(4)$ \\
\hline$C(47)-C(46)-C(45)$ & $123.2(3)$ & $\mathrm{C}(4 \mathrm{~S})-\mathrm{C}(3 \mathrm{~S})-\mathrm{H}(3 \mathrm{SA})$ & 111.2 \\
\hline $\mathrm{C}(47)-\mathrm{C}(46)-\mathrm{H}(46)$ & 118.4 & $\mathrm{C}(2 \mathrm{~S})-\mathrm{C}(3 \mathrm{~S})-\mathrm{H}(3 \mathrm{SA})$ & 111.2 \\
\hline $\mathrm{C}(45)-\mathrm{C}(46)-\mathrm{H}(46)$ & 118.4 & $\mathrm{C}(4 \mathrm{~S})-\mathrm{C}(3 \mathrm{~S})-\mathrm{H}(3 \mathrm{SB})$ & 111.2 \\
\hline $\mathrm{C}(48)-\mathrm{C}(47)-\mathrm{C}(46)$ & $120.1(3)$ & $\mathrm{C}(2 \mathrm{~S})-\mathrm{C}(3 \mathrm{~S})-\mathrm{H}(3 \mathrm{SB})$ & 111.2 \\
\hline $\mathrm{C}(48)-\mathrm{C}(47)-\mathrm{H}(47)$ & 119.9 & $\mathrm{H}(3 \mathrm{SA})-\mathrm{C}(3 \mathrm{~S})-\mathrm{H}(3 \mathrm{SB})$ & 109.1 \\
\hline $\mathrm{C}(46)-\mathrm{C}(47)-\mathrm{H}(47)$ & 119.9 & $\mathrm{O}\left(1 \mathrm{~S}^{\prime}\right)-\mathrm{C}(4 \mathrm{~S})-\mathrm{C}(3 \mathrm{~S})$ & $103.8(8)$ \\
\hline $\mathrm{C}(47)-\mathrm{C}(48)-\mathrm{C}(49)$ & $118.8(3)$ & $\mathrm{C}(3 \mathrm{~S})-\mathrm{C}(4 \mathrm{~S})-\mathrm{O}(1 \mathrm{~S})$ & $110.8(4)$ \\
\hline $\mathrm{C}(47)-\mathrm{C}(48)-\mathrm{H}(48)$ & 120.6 & $\mathrm{C}(3 \mathrm{~S})-\mathrm{C}(4 \mathrm{~S})-\mathrm{H}(4 \mathrm{SA})$ & 109.5 \\
\hline $\mathrm{C}(49)-\mathrm{C}(48)-\mathrm{H}(48)$ & 120.6 & $\mathrm{O}(1 \mathrm{~S})-\mathrm{C}(4 \mathrm{~S})-\mathrm{H}(4 \mathrm{SA})$ & 109.5 \\
\hline $\mathrm{C}(48)-\mathrm{C}(49)-\mathrm{C}(50)$ & $120.0(3)$ & $\mathrm{C}(3 \mathrm{~S})-\mathrm{C}(4 \mathrm{~S})-\mathrm{H}(4 \mathrm{SB})$ & 109.5 \\
\hline $\mathrm{C}(48)-\mathrm{C}(49)-\mathrm{H}(49)$ & 120.0 & $\mathrm{O}(1 \mathrm{~S})-\mathrm{C}(4 \mathrm{~S})-\mathrm{H}(4 \mathrm{SB})$ & 109.5 \\
\hline $\mathrm{C}(50)-\mathrm{C}(49)-\mathrm{H}(49)$ & 120.0 & $\mathrm{H}(4 \mathrm{SA})-\mathrm{C}(4 \mathrm{~S})-\mathrm{H}(4 \mathrm{SB})$ & 108.1 \\
\hline$C(49)-C(50)-C(45)$ & $123.1(3)$ & $\mathrm{C}(8 \mathrm{~S})-\mathrm{O}(2 \mathrm{~S})-\mathrm{C}(5 \mathrm{~S})$ & $102.3(5)$ \\
\hline $\mathrm{C}(49)-\mathrm{C}(50)-\mathrm{H}(50)$ & 118.5 & $\mathrm{C}(6 \mathrm{~S})-\mathrm{C}(5 \mathrm{~S})-\mathrm{O}(2 \mathrm{~S})$ & $105.4(7)$ \\
\hline $\mathrm{C}(45)-\mathrm{C}(50)-\mathrm{H}(50)$ & 118.5 & $\mathrm{C}(6 \mathrm{~S})-\mathrm{C}(5 \mathrm{~S})-\mathrm{O}\left(2 \mathrm{~S}^{\prime}\right)$ & $113.4(8)$ \\
\hline C(39)-B(1)-C(27) & $109.7(2)$ & $\mathrm{C}(6 \mathrm{~S})-\mathrm{C}(5 \mathrm{~S})-\mathrm{H}(5 \mathrm{~S} 1)$ & 110.7 \\
\hline $\mathrm{C}(39)-\mathrm{B}(1)-\mathrm{C}(33)$ & $110.6(2)$ & $\mathrm{O}(2 \mathrm{~S})-\mathrm{C}(5 \mathrm{~S})-\mathrm{H}(5 \mathrm{~S} 1)$ & 110.7 \\
\hline$C(27)-B(1)-C(33)$ & $108.9(2)$ & $\mathrm{C}(6 \mathrm{~S})-\mathrm{C}(5 \mathrm{~S})-\mathrm{H}(5 \mathrm{~S} 2)$ & 110.7 \\
\hline $\mathrm{C}(39)-\mathrm{B}(1)-\mathrm{C}(45)$ & $107.9(2)$ & $\mathrm{O}(2 \mathrm{~S})-\mathrm{C}(5 \mathrm{~S})-\mathrm{H}(5 \mathrm{~S} 2)$ & 110.7 \\
\hline$C(27)-B(1)-C(45)$ & $109.5(2)$ & $\mathrm{H}(5 \mathrm{~S} 1)-\mathrm{C}(5 \mathrm{~S})-\mathrm{H}(5 \mathrm{~S} 2)$ & 108.8 \\
\hline$C(33)-B(1)-C(45)$ & $110.2(2)$ & $C(5 S)-C(6 S)-C(7 S)$ & $105.1(6)$ \\
\hline $\mathrm{C}(1 \mathrm{~S})-\mathrm{O}(1 \mathrm{~S})-\mathrm{C}(4 \mathrm{~S})$ & $101.6(4)$ & $C(5 S)-C(6 S)-H(6 S A)$ & 110.7 \\
\hline $\mathrm{O}(1 \mathrm{~S})-\mathrm{C}(1 \mathrm{~S})-\mathrm{C}(2 \mathrm{~S})$ & $106.4(4)$ & $C(7 S)-C(6 S)-H(6 S A)$ & 110.7 \\
\hline $\mathrm{O}\left(1 \mathrm{~S}^{\prime}\right)-\mathrm{C}(1 \mathrm{~S})-\mathrm{C}(2 \mathrm{~S})$ & $101.6(7)$ & $C(5 S)-C(6 S)-H(6 S B)$ & 110.7 \\
\hline $\mathrm{O}(1 \mathrm{~S})-\mathrm{C}(1 \mathrm{~S})-\mathrm{H}(1 \mathrm{SA})$ & 110.5 & $\mathrm{C}(7 \mathrm{~S})-\mathrm{C}(6 \mathrm{~S})-\mathrm{H}(6 \mathrm{SB})$ & 110.7 \\
\hline $\mathrm{C}(2 \mathrm{~S})-\mathrm{C}(1 \mathrm{~S})-\mathrm{H}(1 \mathrm{SA})$ & 110.5 & $\mathrm{H}(6 \mathrm{SA})-\mathrm{C}(6 \mathrm{~S})-\mathrm{H}(6 \mathrm{SB})$ & 108.8 \\
\hline $\mathrm{O}(1 \mathrm{~S})-\mathrm{C}(1 \mathrm{~S})-\mathrm{H}(1 \mathrm{SB})$ & 110.5 & $C(6 S)-C(7 S)-C(8 S)$ & $104.6(5)$ \\
\hline $\mathrm{C}(2 \mathrm{~S})-\mathrm{C}(1 \mathrm{~S})-\mathrm{H}(1 \mathrm{SB})$ & 110.5 & $\mathrm{C}(6 \mathrm{~S})-\mathrm{C}(7 \mathrm{~S})-\mathrm{H}(7 \mathrm{SA})$ & 110.8 \\
\hline $\mathrm{H}(1 \mathrm{SA})-\mathrm{C}(1 \mathrm{~S})-\mathrm{H}(1 \mathrm{SB})$ & 108.6 & $\mathrm{C}(8 \mathrm{~S})-\mathrm{C}(7 \mathrm{~S})-\mathrm{H}(7 \mathrm{SA})$ & 110.8 \\
\hline$C(1 S)-C(2 S)-C(3 S)$ & $102.2(4)$ & $\mathrm{C}(6 \mathrm{~S})-\mathrm{C}(7 \mathrm{~S})-\mathrm{H}(7 \mathrm{SB})$ & 110.8 \\
\hline$C(1 S)-C(2 S)-H(2 S A)$ & 111.3 & $\mathrm{C}(8 \mathrm{~S})-\mathrm{C}(7 \mathrm{~S})-\mathrm{H}(7 \mathrm{SB})$ & 110.8 \\
\hline$C(3 S)-C(2 S)-H(2 S A)$ & 111.3 & $\mathrm{H}(7 \mathrm{SA})-\mathrm{C}(7 \mathrm{~S})-\mathrm{H}(7 \mathrm{SB})$ & 108.9 \\
\hline $\mathrm{C}(1 \mathrm{~S})-\mathrm{C}(2 \mathrm{~S})-\mathrm{H}(2 \mathrm{SB})$ & 111.3 & $\mathrm{O}(2 \mathrm{~S})-\mathrm{C}(8 \mathrm{~S})-\mathrm{C}(7 \mathrm{~S})$ & $103.5(5)$ \\
\hline $\mathrm{C}(3 \mathrm{~S})-\mathrm{C}(2 \mathrm{~S})-\mathrm{H}(2 \mathrm{SB})$ & 111.3 & $\mathrm{C}(7 \mathrm{~S})-\mathrm{C}(8 \mathrm{~S})-\mathrm{O}\left(2 \mathrm{~S}^{\prime}\right)$ & $106.1(6)$ \\
\hline $\mathrm{H}(2 \mathrm{SA})-\mathrm{C}(2 \mathrm{~S})-\mathrm{H}(2 \mathrm{SB})$ & 109.2 & $\mathrm{O}(2 \mathrm{~S})-\mathrm{C}(8 \mathrm{~S})-\mathrm{H}(8 \mathrm{~S} 1)$ & 111.1 \\
\hline
\end{tabular}




$\begin{array}{llll}\mathrm{C}(7 \mathrm{~S})-\mathrm{C}(8 \mathrm{~S})-\mathrm{H}(8 \mathrm{~S} 1) & 111.1 & \mathrm{H}(10 \mathrm{~A})-\mathrm{C}(10 \mathrm{~S})-\mathrm{H}(10 \mathrm{~B}) & 109.2 \\ \mathrm{O}(2 \mathrm{~S})-\mathrm{C}(8 \mathrm{~S})-\mathrm{H}(8 \mathrm{~S} 2) & 111.1 & \mathrm{C}(12 \mathrm{~S})-\mathrm{C}(11 \mathrm{~S})-\mathrm{C}(10 \mathrm{~S}) & 100.7(4) \\ \mathrm{C}(7 \mathrm{~S})-\mathrm{C}(8 \mathrm{~S})-\mathrm{H}(8 \mathrm{~S} 2) & 111.1 & \mathrm{C}(12 \mathrm{~S})-\mathrm{C}(11 \mathrm{~S})-\mathrm{H}(11 \mathrm{~A}) & 111.6 \\ \mathrm{H}(8 \mathrm{~S} 1)-\mathrm{C}(8 \mathrm{~S})-\mathrm{H}(8 \mathrm{~S} 2) & 109.0 & \mathrm{C}(10 \mathrm{~S})-\mathrm{C}(11 \mathrm{~S})-\mathrm{H}(11 \mathrm{~A}) & 111.6 \\ \mathrm{C}(12 \mathrm{~S})-\mathrm{O}(3 \mathrm{~S})-\mathrm{C}(9 \mathrm{~S}) & 109.2(4) & \mathrm{C}(12 \mathrm{~S})-\mathrm{C}(11 \mathrm{~S})-\mathrm{H}(11 \mathrm{~B}) & 111.6 \\ \mathrm{O}(3 \mathrm{~S})-\mathrm{C}(9 \mathrm{~S})-\mathrm{C}(10 \mathrm{~S}) & 107.2(4) & \mathrm{C}(10 \mathrm{~S})-\mathrm{C}(11 \mathrm{~S})-\mathrm{H}(11 \mathrm{~B}) & 111.6 \\ \mathrm{O}(3 \mathrm{~S})-\mathrm{C}(9 \mathrm{~S})-\mathrm{H}(9 \mathrm{SA}) & 110.3 & \mathrm{H}(11 \mathrm{~A})-\mathrm{C}(11 \mathrm{~S})-\mathrm{H}(11 \mathrm{~B}) & 109.4 \\ \mathrm{C}(10 \mathrm{~S})-\mathrm{C}(9 \mathrm{~S})-\mathrm{H}(9 \mathrm{SA}) & 110.3 & \mathrm{O}(3 \mathrm{~S})-\mathrm{C}(12 \mathrm{~S})-\mathrm{C}(11 \mathrm{~S}) & 106.8(4) \\ \mathrm{O}(3 \mathrm{~S})-\mathrm{C}(9 \mathrm{~S})-\mathrm{H}(9 \mathrm{SB}) & 110.3 & \mathrm{O}(3 \mathrm{~S})-\mathrm{C}(12 \mathrm{~S})-\mathrm{H}(12 \mathrm{~A}) & 110.4 \\ \mathrm{C}(10 \mathrm{~S})-\mathrm{C}(9 \mathrm{~S})-\mathrm{H}(9 \mathrm{SB}) & 110.3 & \mathrm{C}(11 \mathrm{~S})-\mathrm{C}(12 \mathrm{~S})-\mathrm{H}(12 \mathrm{~A}) & 110.4 \\ \mathrm{H}(9 \mathrm{SA})-\mathrm{C}(9 \mathrm{~S})-\mathrm{H}(9 \mathrm{SB}) & 108.5 & \mathrm{O}(3 \mathrm{~S})-\mathrm{C}(12 \mathrm{~S})-\mathrm{H}(12 \mathrm{~B}) & 110.4 \\ \mathrm{C}(9 \mathrm{~S})-\mathrm{C}(10 \mathrm{~S})-\mathrm{C}(11 \mathrm{~S}) & 102.0(4) & \mathrm{C}(11 \mathrm{~S})-\mathrm{C}(12 \mathrm{~S})-\mathrm{H}(12 \mathrm{~B}) & 110.4 \\ \mathrm{C}(9 \mathrm{~S})-\mathrm{C}(10 \mathrm{~S})-\mathrm{H}(10 \mathrm{~A}) & 111.4 & \mathrm{H}(12 \mathrm{~A})-\mathrm{C}(12 \mathrm{~S})-\mathrm{H}(12 \mathrm{~B}) & 108.6 \\ \mathrm{C}(11 \mathrm{~S})-\mathrm{C}(10 \mathrm{~S})-\mathrm{H}(10 \mathrm{~A}) & 111.4 & \mathrm{C}(4 \mathrm{~S})-\mathrm{O}\left(1 \mathrm{~S}^{\prime}\right)-\mathrm{C}(1 \mathrm{~S}) & 114.1(12) \\ \mathrm{C}(9 \mathrm{~S})-\mathrm{C}(10 \mathrm{~S})-\mathrm{H}(10 \mathrm{~B}) & 111.4 & \mathrm{C}(5 \mathrm{~S})-\mathrm{O}\left(2 \mathrm{~S}^{\prime}\right)-\mathrm{C}(8 \mathrm{~S}) & 88.7(9) \\ \mathrm{C}(11 \mathrm{~S})-\mathrm{C}(10 \mathrm{~S})-\mathrm{H}(10 \mathrm{~B}) & 111.4 & & \end{array}$




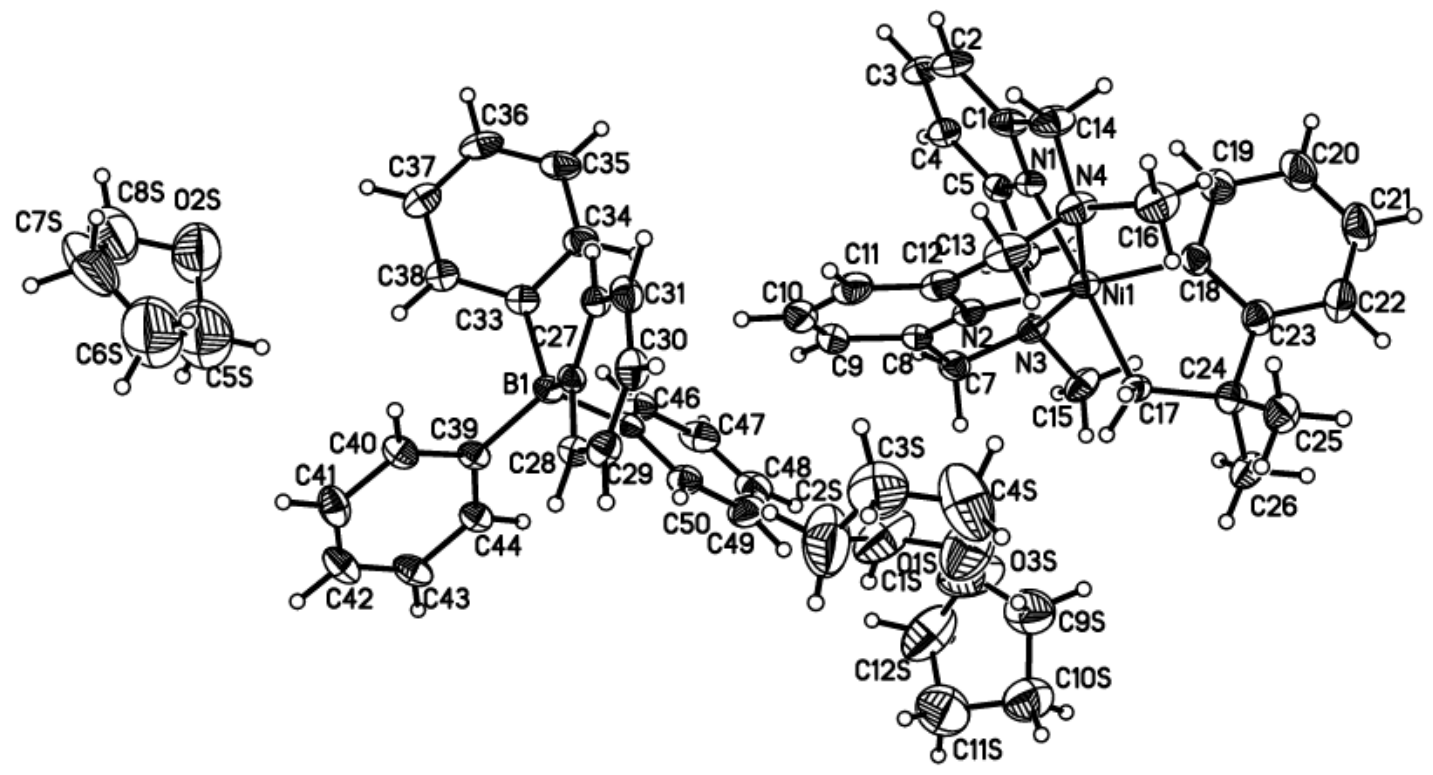

Figure S30. Projection view of $\operatorname{lm} 9315$ with 50\% thermal ellipsoids. 


\section{$\mathrm{X}$-ray structure determination of $\left[\left({ }^{\mathrm{Me}} \mathrm{N} 4\right) \mathrm{Ni}^{\mathrm{II}}(\mathrm{MeCN})_{2}\right]\left(\mathrm{PF}_{6}\right)_{2}$}

Table S27. Crystal data and structure refinement for $\operatorname{lm} 10215$.

Identification code

Empirical formula

Formula weight

Temperature

Wavelength

Crystal system

Space group

Unit cell dimensions

Volume

Z

Density (calculated)

Absorption coefficient

$\mathrm{F}(000)$

Crystal size

Theta range for data collection

Index ranges

Reflections collected

Independent reflections

Completeness to theta $=25.000^{\circ}$

Absorption correction

Max. and min. transmission

Refinement method

Data / restraints / parameters

Goodness-of-fit on $\mathrm{F}^{2}$

Final $\mathrm{R}$ indices $[\mathrm{I}>2 \operatorname{sigma}(\mathrm{I})]$

$\mathrm{R}$ indices (all data)

Extinction coefficient

Largest diff. peak and hole
110215/1t/x8/JS-051915-O2

$\mathrm{C}_{20} \mathrm{H}_{26} \mathrm{~F}_{12} \mathrm{~N}_{6} \mathrm{Ni}_{2}$

699.12

100(2) K

$0.71073 \AA$

Monoclinic

$\mathrm{P} 21 / \mathrm{n}$

$\mathrm{a}=9.2668(5) \AA \quad \alpha=90^{\circ}$.

$\mathrm{b}=16.5937(10) \AA \quad \beta=92.739(3)^{\circ}$.

$\mathrm{c}=18.2485(11) \AA \quad \gamma=90^{\circ}$.

2802.9(3) $\AA^{3}$

4

$1.657 \mathrm{Mg} / \mathrm{m}^{3}$

$0.909 \mathrm{~mm}^{-1}$

1416

$0.374 \times 0.303 \times 0.257 \mathrm{~mm}^{3}$

1.660 to $27.534^{\circ}$.

$-12 \leq \mathrm{h} \leq 10,-18 \leq \mathrm{k} \leq 21,-22 \leq \mathrm{l} \leq 23$

23715

$6328[\mathrm{R}(\mathrm{int})=0.0378]$

$99.3 \%$

Semi-empirical from equivalents

0.8621 and 0.7835

Full-matrix least-squares on $\mathrm{F}^{2}$

6328 / 0 / 374

1.030

$\mathrm{R} 1=0.0663, \mathrm{wR} 2=0.1834$

$\mathrm{R} 1=0.0919, \mathrm{wR} 2=0.2007$

$\mathrm{n} / \mathrm{a}$

1.383 and -1.130 e. $\AA^{-3}$ 
Table S28. Bond lengths $[\AA]$ and angles $\left[{ }^{\circ}\right]$ for $\operatorname{lm} 10215$.

\begin{tabular}{|c|c|c|c|}
\hline $\mathrm{Ni}(1)-\mathrm{N}(2)$ & $2.011(4)$ & $\mathrm{C}(10)-\mathrm{C}(11)$ & $1.377(7)$ \\
\hline $\mathrm{Ni}(1)-\mathrm{N}(1)$ & $2.014(4)$ & $\mathrm{C}(10)-\mathrm{H}(10)$ & 0.9500 \\
\hline $\mathrm{Ni}(1)-\mathrm{N}(5)$ & $2.051(4)$ & $C(11)-C(12)$ & $1.389(7)$ \\
\hline Ni(1)-N(6) & $2.058(4)$ & $\mathrm{C}(11)-\mathrm{H}(11)$ & 0.9500 \\
\hline Ni(1)-N(4) & $2.179(4)$ & $C(12)-C(13)$ & $1.496(7)$ \\
\hline Ni(1)-N(3) & $2.186(4)$ & $\mathrm{C}(13)-\mathrm{H}(13 \mathrm{~A})$ & 0.9900 \\
\hline $\mathrm{N}(1)-\mathrm{C}(1)$ & $1.329(6)$ & $\mathrm{C}(13)-\mathrm{H}(13 \mathrm{~B})$ & 0.9900 \\
\hline $\mathrm{N}(1)-\mathrm{C}(5)$ & $1.347(6)$ & $\mathrm{C}(14)-\mathrm{H}(14 \mathrm{~A})$ & 0.9900 \\
\hline $\mathrm{N}(2)-\mathrm{C}(8)$ & $1.326(6)$ & $\mathrm{C}(14)-\mathrm{H}(14 \mathrm{~B})$ & 0.9900 \\
\hline $\mathrm{N}(2)-\mathrm{C}(12)$ & $1.332(6)$ & $\mathrm{C}(15)-\mathrm{H}(15 \mathrm{~A})$ & 0.9800 \\
\hline $\mathrm{N}(3)-\mathrm{C}(15)$ & $1.478(6)$ & $\mathrm{C}(15)-\mathrm{H}(15 \mathrm{~B})$ & 0.9800 \\
\hline $\mathrm{N}(3)-\mathrm{C}(7)$ & $1.492(6)$ & $\mathrm{C}(15)-\mathrm{H}(15 \mathrm{C})$ & 0.9800 \\
\hline $\mathrm{N}(3)-\mathrm{C}(6)$ & $1.496(6)$ & $C(16)-H(16 A)$ & 0.9800 \\
\hline $\mathrm{N}(4)-\mathrm{C}(16)$ & $1.485(6)$ & $\mathrm{C}(16)-\mathrm{H}(16 \mathrm{~B})$ & 0.9800 \\
\hline $\mathrm{N}(4)-\mathrm{C}(14)$ & $1.489(6)$ & $\mathrm{C}(16)-\mathrm{H}(16 \mathrm{C})$ & 0.9800 \\
\hline $\mathrm{N}(4)-\mathrm{C}(13)$ & $1.494(6)$ & $C(17)-C(18)$ & $1.472(7)$ \\
\hline $\mathrm{N}(5)-\mathrm{C}(17)$ & $1.116(6)$ & $\mathrm{C}(18)-\mathrm{H}(18 \mathrm{~A})$ & 0.9800 \\
\hline $\mathrm{N}(6)-\mathrm{C}(19)$ & $1.130(6)$ & $\mathrm{C}(18)-\mathrm{H}(18 \mathrm{~B})$ & 0.9800 \\
\hline$C(1)-C(2)$ & $1.383(7)$ & $\mathrm{C}(18)-\mathrm{H}(18 \mathrm{C})$ & 0.9800 \\
\hline$C(1)-C(14)$ & $1.497(7)$ & $C(19)-C(20)$ & $1.464(7)$ \\
\hline$C(2)-C(3)$ & $1.397(7)$ & $\mathrm{C}(20)-\mathrm{H}(20 \mathrm{~A})$ & 0.9800 \\
\hline $\mathrm{C}(2)-\mathrm{H}(2)$ & 0.9500 & $\mathrm{C}(20)-\mathrm{H}(20 \mathrm{~B})$ & 0.9800 \\
\hline$C(3)-C(4)$ & $1.386(7)$ & $\mathrm{C}(20)-\mathrm{H}(20 \mathrm{C})$ & 0.9800 \\
\hline $\mathrm{C}(3)-\mathrm{H}(3)$ & 0.9500 & $\mathrm{P}(1)-\mathrm{F}(6)$ & $1.575(3)$ \\
\hline$C(4)-C(5)$ & $1.377(7)$ & $P(1)-F(2)$ & $1.579(3)$ \\
\hline $\mathrm{C}(4)-\mathrm{H}(4)$ & 0.9500 & $\mathrm{P}(1)-\mathrm{F}(1)$ & $1.590(3)$ \\
\hline$C(5)-C(6)$ & $1.501(6)$ & $P(1)-F(5)$ & $1.592(3)$ \\
\hline $\mathrm{C}(6)-\mathrm{H}(6 \mathrm{~A})$ & 0.9900 & $\mathrm{P}(1)-\mathrm{F}(4)$ & $1.595(3)$ \\
\hline $\mathrm{C}(6)-\mathrm{H}(6 \mathrm{~B})$ & 0.9900 & $P(1)-F(3)$ & $1.598(3)$ \\
\hline$C(7)-C(8)$ & $1.515(6)$ & $\mathrm{P}(2)-\mathrm{F}(11)$ & $1.531(5)$ \\
\hline $\mathrm{C}(7)-\mathrm{H}(7 \mathrm{~A})$ & 0.9900 & $\mathrm{P}(2)-\mathrm{F}(7)$ & $1.554(3)$ \\
\hline $\mathrm{C}(7)-\mathrm{H}(7 \mathrm{~B})$ & 0.9900 & $\mathrm{P}(2)-\mathrm{F}(12)$ & $1.577(5)$ \\
\hline$C(8)-C(9)$ & $1.374(7)$ & $\mathrm{P}(2)-\mathrm{F}(9)$ & $1.584(5)$ \\
\hline$C(9)-C(10)$ & $1.379(7)$ & $\mathrm{P}(2)-\mathrm{F}(8)$ & $1.584(4)$ \\
\hline $\mathrm{C}(9)-\mathrm{H}(9)$ & 0.9500 & $P(2)-F(10)$ & $1.596(4)$ \\
\hline
\end{tabular}




\begin{tabular}{|c|c|c|c|}
\hline $\mathrm{N}(2)-\mathrm{Ni}(1)-\mathrm{N}(1)$ & $83.14(15)$ & $\mathrm{N}(1)-\mathrm{C}(1)-\mathrm{C}(14)$ & $114.0(4)$ \\
\hline $\mathrm{N}(2)-\mathrm{Ni}(1)-\mathrm{N}(5)$ & $176.48(16)$ & $\mathrm{C}(2)-\mathrm{C}(1)-\mathrm{C}(14)$ & $124.8(5)$ \\
\hline $\mathrm{N}(1)-\mathrm{Ni}(1)-\mathrm{N}(5)$ & $93.63(15)$ & $\mathrm{C}(1)-\mathrm{C}(2)-\mathrm{C}(3)$ & $118.0(5)$ \\
\hline $\mathrm{N}(2)-\mathrm{Ni}(1)-\mathrm{N}(6)$ & $91.07(15)$ & $\mathrm{C}(1)-\mathrm{C}(2)-\mathrm{H}(2)$ & 121.0 \\
\hline $\mathrm{N}(1)-\mathrm{Ni}(1)-\mathrm{N}(6)$ & $174.05(15)$ & $\mathrm{C}(3)-\mathrm{C}(2)-\mathrm{H}(2)$ & 121.0 \\
\hline $\mathrm{N}(5)-\mathrm{Ni}(1)-\mathrm{N}(6)$ & $92.12(16)$ & $\mathrm{C}(4)-\mathrm{C}(3)-\mathrm{C}(2)$ & $120.2(5)$ \\
\hline $\mathrm{N}(2)-\mathrm{Ni}(1)-\mathrm{N}(4)$ & $81.22(15)$ & $\mathrm{C}(4)-\mathrm{C}(3)-\mathrm{H}(3)$ & 119.9 \\
\hline $\mathrm{N}(1)-\mathrm{Ni}(1)-\mathrm{N}(4)$ & $80.42(15)$ & $\mathrm{C}(2)-\mathrm{C}(3)-\mathrm{H}(3)$ & 119.9 \\
\hline $\mathrm{N}(5)-\mathrm{Ni}(1)-\mathrm{N}(4)$ & $96.89(15)$ & $C(5)-C(4)-C(3)$ & $118.4(4)$ \\
\hline $\mathrm{N}(6)-\mathrm{Ni}(1)-\mathrm{N}(4)$ & $97.39(15)$ & $\mathrm{C}(5)-\mathrm{C}(4)-\mathrm{H}(4)$ & 120.8 \\
\hline $\mathrm{N}(2)-\mathrm{Ni}(1)-\mathrm{N}(3)$ & $80.46(15)$ & $\mathrm{C}(3)-\mathrm{C}(4)-\mathrm{H}(4)$ & 120.8 \\
\hline $\mathrm{N}(1)-\mathrm{Ni}(1)-\mathrm{N}(3)$ & $80.97(15)$ & $\mathrm{N}(1)-\mathrm{C}(5)-\mathrm{C}(4)$ & $121.0(4)$ \\
\hline $\mathrm{N}(5)-\mathrm{Ni}(1)-\mathrm{N}(3)$ & $100.47(15)$ & $\mathrm{N}(1)-\mathrm{C}(5)-\mathrm{C}(6)$ & $113.9(4)$ \\
\hline $\mathrm{N}(6)-\mathrm{Ni}(1)-\mathrm{N}(3)$ & $99.47(15)$ & $C(4)-C(5)-C(6)$ & $125.1(4)$ \\
\hline $\mathrm{N}(4)-\mathrm{Ni}(1)-\mathrm{N}(3)$ & $155.24(14)$ & $\mathrm{N}(3)-\mathrm{C}(6)-\mathrm{C}(5)$ & $112.5(4)$ \\
\hline$C(1)-N(1)-C(5)$ & $121.1(4)$ & $\mathrm{N}(3)-\mathrm{C}(6)-\mathrm{H}(6 \mathrm{~A})$ & 109.1 \\
\hline $\mathrm{C}(1)-\mathrm{N}(1)-\mathrm{Ni}(1)$ & $117.6(3)$ & $\mathrm{C}(5)-\mathrm{C}(6)-\mathrm{H}(6 \mathrm{~A})$ & 109.1 \\
\hline $\mathrm{C}(5)-\mathrm{N}(1)-\mathrm{Ni}(1)$ & $117.6(3)$ & $\mathrm{N}(3)-\mathrm{C}(6)-\mathrm{H}(6 \mathrm{~B})$ & 109.1 \\
\hline $\mathrm{C}(8)-\mathrm{N}(2)-\mathrm{C}(12)$ & $122.1(4)$ & $\mathrm{C}(5)-\mathrm{C}(6)-\mathrm{H}(6 \mathrm{~B})$ & 109.1 \\
\hline $\mathrm{C}(8)-\mathrm{N}(2)-\mathrm{Ni}(1)$ & $117.9(3)$ & $\mathrm{H}(6 \mathrm{~A})-\mathrm{C}(6)-\mathrm{H}(6 \mathrm{~B})$ & 107.8 \\
\hline $\mathrm{C}(12)-\mathrm{N}(2)-\mathrm{Ni}(1)$ & $116.7(3)$ & $\mathrm{N}(3)-\mathrm{C}(7)-\mathrm{C}(8)$ & $110.7(4)$ \\
\hline$C(15)-N(3)-C(7)$ & $108.6(4)$ & $\mathrm{N}(3)-\mathrm{C}(7)-\mathrm{H}(7 \mathrm{~A})$ & 109.5 \\
\hline $\mathrm{C}(15)-\mathrm{N}(3)-\mathrm{C}(6)$ & $108.8(3)$ & $\mathrm{C}(8)-\mathrm{C}(7)-\mathrm{H}(7 \mathrm{~A})$ & 109.5 \\
\hline $\mathrm{C}(7)-\mathrm{N}(3)-\mathrm{C}(6)$ & $111.3(4)$ & $\mathrm{N}(3)-\mathrm{C}(7)-\mathrm{H}(7 \mathrm{~B})$ & 109.5 \\
\hline $\mathrm{C}(15)-\mathrm{N}(3)-\mathrm{Ni}(1)$ & $116.7(3)$ & $\mathrm{C}(8)-\mathrm{C}(7)-\mathrm{H}(7 \mathrm{~B})$ & 109.5 \\
\hline $\mathrm{C}(7)-\mathrm{N}(3)-\mathrm{Ni}(1)$ & $105.3(3)$ & $\mathrm{H}(7 \mathrm{~A})-\mathrm{C}(7)-\mathrm{H}(7 \mathrm{~B})$ & 108.1 \\
\hline $\mathrm{C}(6)-\mathrm{N}(3)-\mathrm{Ni}(1)$ & $106.1(3)$ & $\mathrm{N}(2)-\mathrm{C}(8)-\mathrm{C}(9)$ & $120.8(4)$ \\
\hline$C(16)-N(4)-C(14)$ & $109.5(4)$ & $\mathrm{N}(2)-\mathrm{C}(8)-\mathrm{C}(7)$ & $113.7(4)$ \\
\hline $\mathrm{C}(16)-\mathrm{N}(4)-\mathrm{C}(13)$ & $108.6(4)$ & $C(9)-C(8)-C(7)$ & $125.5(4)$ \\
\hline$C(14)-N(4)-C(13)$ & $111.2(4)$ & $\mathrm{C}(8)-\mathrm{C}(9)-\mathrm{C}(10)$ & $118.0(5)$ \\
\hline $\mathrm{C}(16)-\mathrm{N}(4)-\mathrm{Ni}(1)$ & $116.4(3)$ & $\mathrm{C}(8)-\mathrm{C}(9)-\mathrm{H}(9)$ & 121.0 \\
\hline $\mathrm{C}(14)-\mathrm{N}(4)-\mathrm{Ni}(1)$ & $105.8(3)$ & $\mathrm{C}(10)-\mathrm{C}(9)-\mathrm{H}(9)$ & 121.0 \\
\hline $\mathrm{C}(13)-\mathrm{N}(4)-\mathrm{Ni}(1)$ & $105.2(3)$ & $\mathrm{C}(11)-\mathrm{C}(10)-\mathrm{C}(9)$ & $121.1(5)$ \\
\hline $\mathrm{C}(17)-\mathrm{N}(5)-\mathrm{Ni}(1)$ & $173.1(4)$ & $\mathrm{C}(11)-\mathrm{C}(10)-\mathrm{H}(10)$ & 119.4 \\
\hline $\mathrm{C}(19)-\mathrm{N}(6)-\mathrm{Ni}(1)$ & $169.6(4)$ & $\mathrm{C}(9)-\mathrm{C}(10)-\mathrm{H}(10)$ & 119.4 \\
\hline $\mathrm{N}(1)-\mathrm{C}(1)-\mathrm{C}(2)$ & $121.2(4)$ & $\mathrm{C}(10)-\mathrm{C}(11)-\mathrm{C}(12)$ & $117.8(5)$ \\
\hline
\end{tabular}




\begin{tabular}{|c|c|c|c|}
\hline $\mathrm{C}(10)-\mathrm{C}(11)-\mathrm{H}(11)$ & 121.1 & $\mathrm{~N}(6)-\mathrm{C}(19)-\mathrm{C}(20)$ & $178.9(6)$ \\
\hline $\mathrm{C}(12)-\mathrm{C}(11)-\mathrm{H}(11)$ & 121.1 & $\mathrm{C}(19)-\mathrm{C}(20)-\mathrm{H}(20 \mathrm{~A})$ & 109.5 \\
\hline $\mathrm{N}(2)-\mathrm{C}(12)-\mathrm{C}(11)$ & $120.1(4)$ & $\mathrm{C}(19)-\mathrm{C}(20)-\mathrm{H}(20 \mathrm{~B})$ & 109.5 \\
\hline $\mathrm{N}(2)-\mathrm{C}(12)-\mathrm{C}(13)$ & $114.2(4)$ & $\mathrm{H}(20 \mathrm{~A})-\mathrm{C}(20)-\mathrm{H}(20 \mathrm{~B})$ & 109.5 \\
\hline$C(11)-C(12)-C(13)$ & $125.6(4)$ & $C(19)-C(20)-H(20 C)$ & 109.5 \\
\hline $\mathrm{N}(4)-\mathrm{C}(13)-\mathrm{C}(12)$ & $111.7(4)$ & $\mathrm{H}(20 \mathrm{~A})-\mathrm{C}(20)-\mathrm{H}(20 \mathrm{C})$ & 109.5 \\
\hline $\mathrm{N}(4)-\mathrm{C}(13)-\mathrm{H}(13 \mathrm{~A})$ & 109.3 & $\mathrm{H}(20 \mathrm{~B})-\mathrm{C}(20)-\mathrm{H}(20 \mathrm{C})$ & 109.5 \\
\hline$C(12)-C(13)-H(13 A)$ & 109.3 & $F(6)-P(1)-F(2)$ & $91.5(2)$ \\
\hline $\mathrm{N}(4)-\mathrm{C}(13)-\mathrm{H}(13 \mathrm{~B})$ & 109.3 & $\mathrm{~F}(6)-\mathrm{P}(1)-\mathrm{F}(1)$ & $90.10(19)$ \\
\hline $\mathrm{C}(12)-\mathrm{C}(13)-\mathrm{H}(13 \mathrm{~B})$ & 109.3 & $F(2)-P(1)-F(1)$ & $91.6(2)$ \\
\hline $\mathrm{H}(13 \mathrm{~A})-\mathrm{C}(13)-\mathrm{H}(13 \mathrm{~B})$ & 107.9 & $\mathrm{~F}(6)-\mathrm{P}(1)-\mathrm{F}(5)$ & $179.3(2)$ \\
\hline $\mathrm{N}(4)-\mathrm{C}(14)-\mathrm{C}(1)$ & $111.4(4)$ & $\mathrm{F}(2)-\mathrm{P}(1)-\mathrm{F}(5)$ & $88.8(2)$ \\
\hline $\mathrm{N}(4)-\mathrm{C}(14)-\mathrm{H}(14 \mathrm{~A})$ & 109.3 & $\mathrm{~F}(1)-\mathrm{P}(1)-\mathrm{F}(5)$ & $89.23(18)$ \\
\hline $\mathrm{C}(1)-\mathrm{C}(14)-\mathrm{H}(14 \mathrm{~A})$ & 109.3 & $\mathrm{~F}(6)-\mathrm{P}(1)-\mathrm{F}(4)$ & $90.43(19)$ \\
\hline $\mathrm{N}(4)-\mathrm{C}(14)-\mathrm{H}(14 \mathrm{~B})$ & 109.3 & $\mathrm{~F}(2)-\mathrm{P}(1)-\mathrm{F}(4)$ & $177.6(2)$ \\
\hline $\mathrm{C}(1)-\mathrm{C}(14)-\mathrm{H}(14 \mathrm{~B})$ & 109.3 & $\mathrm{~F}(1)-\mathrm{P}(1)-\mathrm{F}(4)$ & $89.82(19)$ \\
\hline $\mathrm{H}(14 \mathrm{~A})-\mathrm{C}(14)-\mathrm{H}(14 \mathrm{~B})$ & 108.0 & $\mathrm{~F}(5)-\mathrm{P}(1)-\mathrm{F}(4)$ & $89.30(19)$ \\
\hline $\mathrm{N}(3)-\mathrm{C}(15)-\mathrm{H}(15 \mathrm{~A})$ & 109.5 & $\mathrm{~F}(6)-\mathrm{P}(1)-\mathrm{F}(3)$ & $90.13(18)$ \\
\hline $\mathrm{N}(3)-\mathrm{C}(15)-\mathrm{H}(15 \mathrm{~B})$ & 109.5 & $\mathrm{~F}(2)-\mathrm{P}(1)-\mathrm{F}(3)$ & $89.2(2)$ \\
\hline $\mathrm{H}(15 \mathrm{~A})-\mathrm{C}(15)-\mathrm{H}(15 \mathrm{~B})$ & 109.5 & $\mathrm{~F}(1)-\mathrm{P}(1)-\mathrm{F}(3)$ & $179.2(2)$ \\
\hline $\mathrm{N}(3)-\mathrm{C}(15)-\mathrm{H}(15 \mathrm{C})$ & 109.5 & $\mathrm{~F}(5)-\mathrm{P}(1)-\mathrm{F}(3)$ & $90.53(18)$ \\
\hline $\mathrm{H}(15 \mathrm{~A})-\mathrm{C}(15)-\mathrm{H}(15 \mathrm{C})$ & 109.5 & $\mathrm{~F}(4)-\mathrm{P}(1)-\mathrm{F}(3)$ & $89.36(19)$ \\
\hline $\mathrm{H}(15 \mathrm{~B})-\mathrm{C}(15)-\mathrm{H}(15 \mathrm{C})$ & 109.5 & $\mathrm{~F}(11)-\mathrm{P}(2)-\mathrm{F}(7)$ & $92.7(3)$ \\
\hline N(4)-C(16)-H(16A) & 109.5 & $\mathrm{~F}(11)-\mathrm{P}(2)-\mathrm{F}(12)$ & 174.6(3) \\
\hline $\mathrm{N}(4)-\mathrm{C}(16)-\mathrm{H}(16 \mathrm{~B})$ & 109.5 & $\mathrm{~F}(7)-\mathrm{P}(2)-\mathrm{F}(12)$ & $91.9(2)$ \\
\hline $\mathrm{H}(16 \mathrm{~A})-\mathrm{C}(16)-\mathrm{H}(16 \mathrm{~B})$ & 109.5 & $\mathrm{~F}(11)-\mathrm{P}(2)-\mathrm{F}(9)$ & $86.1(3)$ \\
\hline $\mathrm{N}(4)-\mathrm{C}(16)-\mathrm{H}(16 \mathrm{C})$ & 109.5 & $\mathrm{~F}(7)-\mathrm{P}(2)-\mathrm{F}(9)$ & 178.2(3) \\
\hline $\mathrm{H}(16 \mathrm{~A})-\mathrm{C}(16)-\mathrm{H}(16 \mathrm{C})$ & 109.5 & $\mathrm{~F}(12)-\mathrm{P}(2)-\mathrm{F}(9)$ & $89.4(3)$ \\
\hline $\mathrm{H}(16 \mathrm{~B})-\mathrm{C}(16)-\mathrm{H}(16 \mathrm{C})$ & 109.5 & $\mathrm{~F}(11)-\mathrm{P}(2)-\mathrm{F}(8)$ & $94.3(3)$ \\
\hline $\mathrm{N}(5)-\mathrm{C}(17)-\mathrm{C}(18)$ & 179.1(6) & $\mathrm{F}(7)-\mathrm{P}(2)-\mathrm{F}(8)$ & $91.4(2)$ \\
\hline $\mathrm{C}(17)-\mathrm{C}(18)-\mathrm{H}(18 \mathrm{~A})$ & 109.5 & $\mathrm{~F}(12)-\mathrm{P}(2)-\mathrm{F}(8)$ & $88.6(3)$ \\
\hline $\mathrm{C}(17)-\mathrm{C}(18)-\mathrm{H}(18 \mathrm{~B})$ & 109.5 & $\mathrm{~F}(9)-\mathrm{P}(2)-\mathrm{F}(8)$ & $87.4(2)$ \\
\hline $\mathrm{H}(18 \mathrm{~A})-\mathrm{C}(18)-\mathrm{H}(18 \mathrm{~B})$ & 109.5 & $\mathrm{~F}(11)-\mathrm{P}(2)-\mathrm{F}(10)$ & $89.7(3)$ \\
\hline $\mathrm{C}(17)-\mathrm{C}(18)-\mathrm{H}(18 \mathrm{C})$ & 109.5 & $\mathrm{~F}(7)-\mathrm{P}(2)-\mathrm{F}(10)$ & $91.4(2)$ \\
\hline $\mathrm{H}(18 \mathrm{~A})-\mathrm{C}(18)-\mathrm{H}(18 \mathrm{C})$ & 109.5 & $\mathrm{~F}(12)-\mathrm{P}(2)-\mathrm{F}(10)$ & $87.2(3)$ \\
\hline $\mathrm{H}(18 \mathrm{~B})-\mathrm{C}(18)-\mathrm{H}(18 \mathrm{C})$ & 109.5 & $\mathrm{~F}(9)-\mathrm{P}(2)-\mathrm{F}(10)$ & $90.0(2)$ \\
\hline
\end{tabular}


$F(8)-P(2)-F(10)$

175.1(3) 

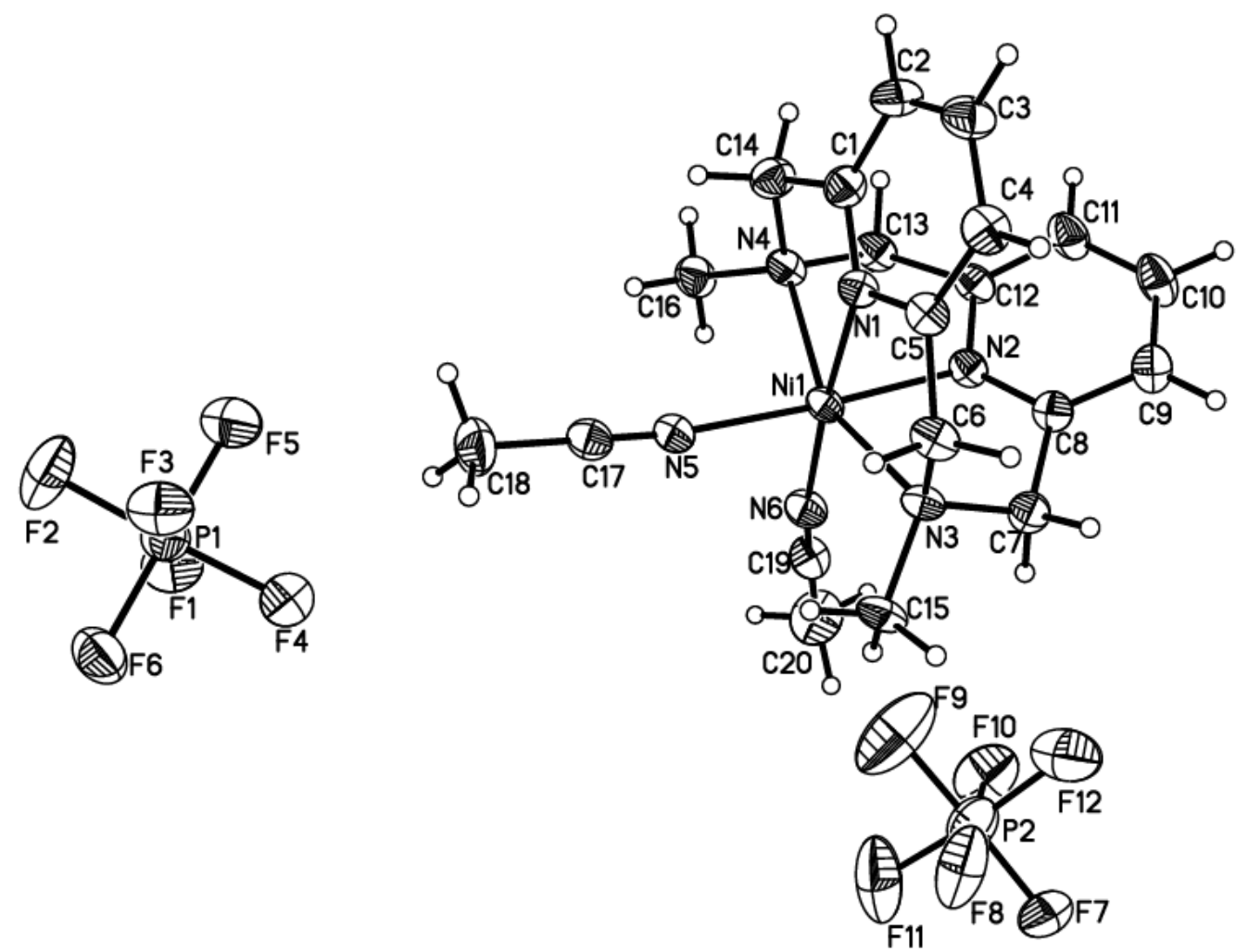

Figure S31. Projection view of $\operatorname{lm} 10215$ with 50\% thermal ellipsoids. 


\section{References}

1. Khusnutdinova, J. R.; Rath, N. P.; Mirica, L. M. J. Am. Chem. Soc. 2012, 134, 2414.

2. Bottino, F.; Di Grazia, M.; Finocchiaro, P.; Fronczek, F. R.; Mamo, A.; Pappalardo, S. J. Org. Chem. 1988, 53, 3521.

3. Carmona, E.; Gutierrezpuebla, E.; Marin, J. M.; Monge, A.; Paneque, M.; Poveda, M. L.; Ruiz, C. J. Am. Chem. Soc. 1989, 111, 2883.

4. Connelly, N. G.; Geiger, W. E. Chem. Rev. 1996, 96, 877.

5. Gottlieb, H. E.; Kotlyar, V.; Nudelman, A. J. Org. Chem. 1997, 62, 7512.

6. M. J. Frisch, G. W. T., H. B. Schlegel, G. E. Scuseria, M. A. Robb, J. R. Cheeseman, G. Scalmani, V. Barone, B. Mennucci, G. A. Petersson, H. Nakatsuji, M. Caricato, X. Li, H. P. Hratchian, A. F. Izmaylov, J. Bloino, G. Zheng, J. L. Sonnenberg, M. Hada, M. Ehara, K. Toyota, R. Fukuda, J. Hasegawa, M. Ishida, T. Nakajima, Y. Honda, O. Kitao, H. Nakai, T. Vreven, J. A. Montgomery, Jr., J. E. Peralta, F. Ogliaro, M. Bearpark, J. J. Heyd, E. Brothers, K. N. Kudin, V. N. Staroverov, R. Kobayashi, J. Normand, K. Raghavachari, A. Rendell, J. C. Burant, S. S. Iyengar, J. Tomasi, M. Cossi, N. Rega, J. M. Millam, M. Klene, J. E. Knox, J. B. Cross, V. Bakken, C. Adamo, J. Jaramillo, R. Gomperts, R. E. Stratmann, O. Yazyev, A. J. Austin, R. Cammi, C. Pomelli, J. W. Ochterski, R. L. Martin, K. Morokuma, V. G. Zakrzewski, G. A. Voth, P. Salvador, J. J. Dannenberg, S. Dapprich, A. D. Daniels, O. Farkas, J. B. Foresman, J. V. Ortiz, J. Cioslowski, D. J. Fox, Gaussian 09, Revision A.02, Gaussian, Inc., Wallingford CT, 2009.

7. Lee, C. M.; Chen, C. H.; Liao, F. X.; Hu, C. H.; Lee, G. H. J. Am. Chem. Soc. 2010, 132, 9256.

8. Zhang, C. P.; Wang, H.; Klein, A.; Biewer, C.; Stimat, K.; Yarnaguchi, Y.; Xu, L.; GomezBenitez, V.; Vicic, D. A. J. Am. Chem. Soc. 2013, 135, 8141.

9. Skripnikov, L. Chemissian, version 1.771, 2010, www.chemissian.com, accessed June 2011, 